S1 Table. Characteristics of included studies

Bailey 2010

\begin{tabular}{|c|c|}
\hline Methods & $\begin{array}{l}\text { 24-week phase 3, multicenter, randomized, placebo-controlled, } \\
\text { double-blind, parallel-group trial in } 80 \text { sites ( } 30 \text { in the USA, } 21 \text { in } \\
\text { Canada, } 11 \text { in Argentina, ten in Mexico, and eight in Brazil). }\end{array}$ \\
\hline Participants & $\begin{array}{l}546 \text { initially randomized: dapagliflozin } 2 \cdot 5 \mathrm{mg} n=137 \text {; dapagliflozin } 5 \\
m g \mathrm{n}=137 \text {; dapagliflozin } 10 \mathrm{mg} n=135 ; \text { placebo } n=137 \text {. }\end{array}$ \\
\hline Interventions & $\begin{array}{l}\text { Dapagliflozin } 2.5 \mathrm{mg} \text {; dapagliflozin } 5 \mathrm{mg} \text {; dapagliflozin } 5 \mathrm{mg} \mathrm{n}=135 \text {; } \\
\text { placebo } \mathrm{n}=137 . \\
\text { Randomly assigned (in a 1:1:1:1 ratio) by the IVRS to double-blinded } \\
\text { groups of once-daily dapagliflozin } 2.5 \mathrm{mg}, 5 \mathrm{mg} \text {, or } 10 \mathrm{mg} \text {, or } \\
\text { matching placebo given orally before the morning meal for } 24 \\
\text { weeks. } \\
\text { Add on: metformin open-label } 500 \mathrm{mg} \text { metformin, diet and exercise } \\
\text { counselling. }\end{array}$ \\
\hline Outcomes & $\begin{array}{l}\text { Trial extended to } 102 \text { weeks, outcome data derived from } 102 \text { weeks. } \\
\text { Primary outcomes of the trial } \\
\text { change in HbA1c levels from baseline at week } 102 . \\
\text { Secondary outcomes of the trial } \\
\text { change from baseline in fasting plasma glucose (FPG) level } \\
\text { percent change from baseline in body weight change and } \\
\text { decreases in bodyweight of } 5 \% \text { or more. } \\
\text { changes in fasting plasma glucose concentration and total } \\
\text { bodyweight at week } 24, \\
\text { change in fasting plasma glucose concentration at week } 1, \\
\text { the proportion of patients achieving a therapeutic glycemic } \\
\text { response (HbA1c <7\% at week } 24 \text { ) } \\
\text { lipids; total cholesterol, LDL cholesterol. } \\
\text { Adverse events and safety assessments } \\
\text { adverse events (AEs) were reported throughout the study, } \\
\text { urinary tract infection, genital infection. } \\
\text { Serious adverse events defined as: } \\
\text { fatal, life threatening, }\end{array}$ \\
\hline
\end{tabular}




\begin{tabular}{|c|c|}
\hline & $\begin{array}{l}\text { required admission to hospital, } \\
\text { prolonged an existing hospital stay, } \\
\text { resulted in persistent or significant disability or incapacity, } \\
\text { cancer or a congenital anomaly, } \\
\text { resulted in development of drug dependency or drug abuse, } \\
\text { medical event that jeopardized the patient or required } \\
\text { intervention to prevent a serious outcome. }\end{array}$ \\
\hline Inclusion criteria & $\begin{array}{l}\text { men and women, } \\
\text { T2DM, } \\
\text { aged } 18-77 \text { years, } \\
\text { with inadequate glycemic control ( } \mathrm{HbA} 1 \mathrm{c} \text { levels } 7-10 \%) \text {, } \\
\text { C-peptide concentration } 0 \cdot 34 \mathrm{nmol} / \mathrm{L} \text { or more, } \\
\text { body-mass index } 45 \mathrm{~kg} / \mathrm{m}^{2} \text { or less, } \\
\text { taking a stable dose of metformin ( } \geq 1500 \mathrm{mg} \text { per day) for at least } 8 \\
\text { weeks before enrolment. }\end{array}$ \\
\hline Exclusion criteria & $\begin{array}{l}\text { serum creatinine } 133 \mu \mathrm{mol} / \mathrm{L} \text { or more for men or } 124 \mu \mathrm{mol} / \mathrm{L} \text { or } \\
\text { more for women (consistent with metformin labelling), } \\
\text { urine albumin/creatinine ratio more than } 203.4 \mathrm{mg} / \mathrm{mmol} \text {, } \\
\text { aspartate aminotransferase or alanine aminotransferase more than } \\
\text { three times the upper limit of normal, } \\
\text { creatine kinase more than three times the upper limit of normal, } \\
\text { symptoms of poorly controlled diabetes (including marked polyuria } \\
\text { and polydipsia with }>10 \% \text { weight loss during the } 3 \text { months before } \\
\text { enrolment), } \\
\text { clinically significant renal, hepatic, hematological, oncological, } \\
\text { endocrine, psychiatric, or rheumatic disease; recent cardiovascular } \\
\text { event (within } 6 \text { months) or New York Heart Association } \\
\text { class III or IV congestive heart failure, } \\
\text { systolic blood pressure } 180 \mathrm{~mm} \text { Hg or more or diastolic blood } \\
\text { pressure } 110 \mathrm{~mm} \text { Hg or more. }\end{array}$ \\
\hline \multirow[t]{2}{*}{ Notes } & ClinicalTrials.gov identifier: NCT00528879 \\
\hline & $\begin{array}{l}\text { Background treatment metformin. 2010, } 24 \text { weeks. Bailey } 2013 \text { is a } \\
78 \text {-week extension of Bailey } 2010 . \text { We use outcome data from } 102 \\
\text { weeks in our analyses. }\end{array}$ \\
\hline
\end{tabular}

Risk of bias table

\begin{tabular}{||l|l|l||}
\hline Bias & $\begin{array}{l}\text { Authors' } \\
\text { judgement }\end{array}$ & Support for judgement \\
\hline \hline Random sequence & Low risk & Random numbers by central interactive voice response \\
\hline
\end{tabular}




\begin{tabular}{|c|c|c|}
\hline $\begin{array}{l}\text { generation } \\
\text { (selection bias) }\end{array}$ & & $\begin{array}{l}\text { system. Site was assigned a block of random patient } \\
\text { treatment assignments when calling to randomize the } \\
\text { site's first patient. }\end{array}$ \\
\hline $\begin{array}{l}\text { Allocation } \\
\text { concealment } \\
\text { (selection bias) }\end{array}$ & Low risk & $\begin{array}{l}\text { Randomization schedules were computer-generated by } \\
\text { the sponsor and stored in a secure location. }\end{array}$ \\
\hline $\begin{array}{l}\text { Blinding of } \\
\text { participants and } \\
\text { personnel } \\
\text { (performance bias) }\end{array}$ & Low risk & $\begin{array}{l}\text { The patients, investigators, and sponsor personnel were } \\
\text { blinded to treatment allocation and HbA1c and urinary } \\
\text { glucose concentrations. The film-coated placebo and } \\
\text { active tablets were similar in color, shape, size, texture, } \\
\text { and taste. }\end{array}$ \\
\hline $\begin{array}{l}\text { Blinding of outcome } \\
\text { assessment } \\
\text { (detection bias) }\end{array}$ & Low risk & $\begin{array}{l}\text { The patients, investigators, and sponsor personnel were } \\
\text { blinded to treatment allocation and HbA1c and urinary } \\
\text { glucose concentrations. The film-coated placebo and } \\
\text { active tablets were similar in color, shape, size, texture, } \\
\text { and taste. }\end{array}$ \\
\hline $\begin{array}{l}\text { Incomplete } \\
\text { outcome data } \\
\text { (attrition bias) }\end{array}$ & Low risk & $\begin{array}{l}483 / 546 \text { completed. Data set comprised } n=534: \text { i.e. all } \\
\text { randomized patients who received at least one dose of } \\
\text { double-blind study medication and who had both a } \\
\text { baseline and at } \\
\text { least one post-baseline measurement. Last observation } \\
\text { carried forward, drops and losses to follow up all } \\
\text { accounted for. }\end{array}$ \\
\hline $\begin{array}{l}\text { Selective reporting } \\
\text { (reporting bias) }\end{array}$ & Low risk & $\begin{array}{l}\text { All clinically relevant outcomes were defined and } \\
\text { reported. }\end{array}$ \\
\hline Other bias & Unclear risk & $\begin{array}{l}\text { The sponsors of the study were involved in study design, } \\
\text { data collection, data review, and data analysis, and were } \\
\text { responsible for gathering all data from investigation } \\
\text { centers for the clinical database. The report was prepared } \\
\text { by the authors, with contributions from the sponsor } \\
\text { Bristol-Myers Squibb. }\end{array}$ \\
\hline
\end{tabular}

Bode 2013

\begin{tabular}{||l|l||}
\hline Methods & $\begin{array}{l}26 \text { week (extended to } 104 \text { weeks), randomized, double-blind, } \\
\text { placebo-controlled, phase } 3 \text { study in } 90 \text { study centers in } 17 \text { countries }\end{array}$ \\
\hline Participants & $\begin{array}{l}714 \text { initially randomized: canagliflozin 100mg n=241; canagliflozin } \\
300 m g ~ n=236 ; \text { placebo } n=237 .\end{array}$ \\
\hline
\end{tabular}




\begin{tabular}{|c|c|}
\hline Interventions & $\begin{array}{l}\text { Canagliflozin } 300 \mathrm{mg} \text { or } 300 \mathrm{mg} \text { versus placebo for up to } 104 \text { weeks. } \\
\text { Participants were randomized in a I:I:I ratio to canagliflozin } 100 \mathrm{mg} \\
\text { or } 300 \mathrm{mg} \text { or placebo once daily before the first meal of the day as } \\
\text { add-on therapy to their ongoing stable T2DM treatment regimen. } \\
\text { Add on: metformin, alfaglucosidase, DPP-4, GLP1-a, insulin, } \\
\text { pioglitazone, SU. }\end{array}$ \\
\hline Outcomes & $\begin{array}{l}\text { Primary outcomes of the trial } \\
\text { change in HbA1c levels from baseline to week 26, outcome data in } \\
\text { our analyses are derived from the 104-week time point (extension } \\
\text { study). } \\
\text { Secondary outcomes of the trial } \\
\text { change from baseline in fasting plasma glucose (FPG) level, } \\
\text { systolic blood pressure (BP), } \\
\text { percent change from baseline in body weight, } \\
\text { triglyceride levels, } \\
\text { high-density lipoprotein cholesterol (HDL-C) level, } \\
\text { proportion reaching HbA1c levels < 7.0\%. } \\
\text { Adverse events and safety assessments } \\
\text { adverse events, } \\
\text { safety laboratory tests, } \\
\text { vital sign measurements, } \\
\text { physical examinations and } 12-l e a d \text { electrocardiograms, } \\
\text { osmotic diuresis and volume depletion, } \\
\text { urinary tract infections (UTIs), } \\
\text { genital mycotic infections. }\end{array}$ \\
\hline Inclusion criteria & $\begin{array}{l}\text { men and women } \\
\text { T2DM } \\
\text { aged } 55 \text { to } 80 \text { years, (older adults) } \\
\text { BMI between } 20 \text { and } 40 \mathrm{~kg} / \mathrm{m}^{2} \text {, } \\
\text { FPG level }<270 \mathrm{mg} / \mathrm{dL}(15.0 \mathrm{mmol} / \mathrm{L} \text { ) at start of the single-blind, } \\
\text { placebo run-in period), } \\
\text { fasting fingerstick blood glucose level } 2: 110 \mathrm{mg} / \mathrm{dL}(6.1 \mathrm{mmol} / \mathrm{L} \text { ) } \\
\text { and }<270 \mathrm{mg} / \mathrm{dL} \text { ( } 15.0 \mathrm{mmol} / \mathrm{L} \text { ) at baseline. } \\
\text { with inadequate glycemic control (HbA1c levels } \geq 7.0 \%) \\
\text { on no blood glucose-lowering agent; } \\
\text { or on a stable regimen of blood glucose- lowering agent(s) as } \\
\text { monotherapy or combination therapy (including metformin, } \\
\text { sulfonylurea, dipeptidyl peptidase-4 (DPP-4) inhibitor, a- }\end{array}$ \\
\hline
\end{tabular}




\begin{tabular}{|c|c|}
\hline & $\begin{array}{l}\text { glucosidase inhibitor, glucagon-like peptide-I (GLP-I) agonist, } \\
\text { or insulin for 2: } 12 \text { weeks prior to screening) or pioglitazone for 2: } 6 \\
\text { months prior to screening)) used in accordance with local } \\
\text { prescribing information. }\end{array}$ \\
\hline Exclusion criteria & $\begin{array}{l}\text { type I diabetes mellitus; repeated FPG level } 2: 270 \mathrm{mg} / \mathrm{dL} \text { ( } 15.0 \\
\mathrm{mmol} / \mathrm{L} \text { ) during the pretreatment phase, } \\
\text { history of myocardial infarction, unstable angina, revascularization } \\
\text { procedure, or cerebrovascular accident within } 3 \text { months before } \\
\text { screening; history of New York Heart Association Class II I- IV } \\
\text { cardiac disease, } \\
\text { uncontrolled hypertension; } \\
\text { eGFR }<50 \mathrm{~mL} / \mathrm{min} / 1.73 \mathrm{~m}^{2} \\
\text { if on metformin therapy, excluded if serum creatinine levels } \geq 1.4 \\
\mathrm{mg} / \mathrm{dL} \text { ( } 124 \mu \mathrm{mol} / \mathrm{L} \text { for men and } \geq 1.3 \mathrm{mg} / \mathrm{dL} \text { ( } 115 \mu \mathrm{mol} / \mathrm{L} \text { for } \\
\text { women or any contraindication to the use of metformin (including } \\
\text { low eGFR). }\end{array}$ \\
\hline Notes & $\begin{array}{l}\text { ClinicalTrials.gov identifier: NCT01106651 } \\
\text { Followed by a 78-week, placebo-controlled, double-blind extension } \\
\text { period and a 30-day follow-up period (Bode 2015), data from which } \\
\text { is included in our analyses. }\end{array}$ \\
\hline
\end{tabular}

\section{Risk of bias table}

\begin{tabular}{||l||l|l||}
\hline Bias & $\begin{array}{l}\text { Authors' } \\
\text { judgement }\end{array}$ & Support for judgement \\
\hline $\begin{array}{l}\text { Random sequence } \\
\text { generation } \\
\text { (selection bias) }\end{array}$ & Low risk & $\begin{array}{l}\text { Randomization was performed using an interactive voice } \\
\text { response system/interactive web response system with a } \\
\text { computer-generated randomization schedule prepared by } \\
\text { the sponsor before the study. Randomization carried out } \\
\text { in permuted blocks of } 6 \text { stratified based on T score of } \\
\text { lumbar spine. }\end{array}$ \\
\hline $\begin{array}{l}\text { Allocation } \\
\text { concealment } \\
\text { (selection bias) }\end{array}$ & Unclear risk & $\begin{array}{l}\text { Not described but implied as randomization was } \\
\text { performed using an interactive voice response } \\
\text { system/interactive web response system with a } \\
\text { computer-generated randomization schedule prepared by } \\
\text { the sponsor before the study. }\end{array}$ \\
\hline $\begin{array}{l}\text { Blinding of } \\
\text { participants and } \\
\text { personnel }\end{array}$ & Low risk & $\begin{array}{l}\text { Participants, investigators, and local sponsor personnel } \\
\text { were to remain blinded to treatment assignment until the } \\
\text { final database lock. }\end{array}$ \\
\hline
\end{tabular}




\begin{tabular}{|c|c|c|}
\hline (performance bias) & & $\begin{array}{l}\text { (To maintain blinding after randomization, } \mathrm{HbA} 1 \mathrm{c} \text { and FPG } \\
\text { levels were masked to the study centers unless these } \\
\text { values met prespecified glycemic criteria for the initiation } \\
\text { of rescue medication or after glycemic rescue medication } \\
\text { was started). }\end{array}$ \\
\hline $\begin{array}{l}\text { Blinding of } \\
\text { outcome } \\
\text { assessment } \\
\text { (detection bias) }\end{array}$ & Low risk & $\begin{array}{l}\text { Participants, investigators, and local sponsor personnel } \\
\text { were to remain blinded to treatment assignment until the } \\
\text { final database lock. }\end{array}$ \\
\hline $\begin{array}{l}\text { Incomplete } \\
\text { outcome data } \\
\text { (attrition bias) }\end{array}$ & Low risk & $\begin{array}{l}\text { Completers at } 26 \text { weeks: } 197 / 237 \text { placebo, } 226 / 241 \\
\text { 100mg canagliflozin, 209/236 300mg canagliflozin. } \\
\text { Completers at } 78 \text { weeks: } 158 / 237 \text { placebo, } 184 / 241 \\
100 m g \text { canagliflozin, } 178 / 236300 \mathrm{mg} \text { canagliflozin. } \\
\text { Primary and secondary efficacy analyses were performed } \\
\text { using the modified intent-to-treat (m ITT) population, } \\
\text { which consisted of all randomized participants who took } \\
\text { at least one dose of double-blind study drug ( } \mathrm{n}=714 \text { ). } \\
\text { Drop outs and losses to follow up accounted for. } \\
\text { Study report describes some limitations, in that placebo } \\
\text { participants may have dropped out due to lack of } \\
\text { glycemic control and too few >75 years participants were } \\
\text { included in the trial. }\end{array}$ \\
\hline $\begin{array}{l}\text { Selective reporting } \\
\text { (reporting bias) }\end{array}$ & Low risk & $\begin{array}{l}\text { All major and clinically relevant outcomes were reported. } \\
\text { Minor discrepancies between outcome data in main study } \\
\text { reports and supplementary. Comment: not though to } \\
\text { introduce a source of bias. }\end{array}$ \\
\hline Other bias & Unclear risk & $\begin{array}{l}\text { Data-analysis and editorial support was provided by } \\
\text { pharmaceutical company support, in addition to trial } \\
\text { being carried out by pharmaceutical company. }\end{array}$ \\
\hline
\end{tabular}

Bolinder 2012

\begin{tabular}{||l|l||}
\hline Methods & $\begin{array}{l}\text { 24-week, multicenter, randomized, parallel-group, double-blind, } \\
\text { placebo-controlled phase } 3 \text { study at } 40 \text { sites in } 5 \text { countries (Bulgaria, } \\
\text { Czech Republic, Hungary, Poland, and Sweden). }\end{array}$ \\
\hline Participants & $\begin{array}{l}\text { 446 initially randomized (314 to main assessment, } 132 \text { to sub study): } \\
\text { dapagliflozin 10mg } n=91 ; \text { placebo } n=91 .\end{array}$ \\
\hline
\end{tabular}




\begin{tabular}{|c|c|}
\hline Interventions & $\begin{array}{l}\text { Patients were randomized in a 1:1 ratio to double-blind treatment } \\
\text { with either dapagliflozin } 10 \mathrm{mg} \text { or placebo for } 24 \text { weeks extended to } \\
102 \text { weeks. } \\
\text { Add on: metformin. } \\
\text { Rescue therapy permitted. }\end{array}$ \\
\hline Outcomes & $\begin{array}{l}\text { At } 24 \text { weeks: } \\
\text { Primary outcomes of the trial } \\
\text { change from baseline at week } 24 \text { in total body weight (TBW) } \\
\text { Secondary outcomes of the trial } \\
\text { change from baseline in waist circumference, } \\
\text { total FM as measured by DXA } \\
\text { proportion of patients achieving a body weight reduction of at } \\
\text { least } 5 \% \text { at week } 24 . \\
\text { change from baseline } 24 \text { for total LM as measured by DXA, } \\
\text { glycemic variables } \\
\text { HbA1c } \\
\text { FPG, } \\
\text { adipose tissue markers adiponectin and leptin, } \\
\text { seated systolic and diastolic blood pressure. } \\
\text { MR assessments were conducted in } 80 \text { in the sub study: change } \\
\text { from baseline at week } 24 \text { for visceral adipose tissue (VAT) volume, } \\
\text { adipose tissue (SAT) volume, and hepatic lipid (HL) content. } \\
\text { Adverse events and safety assessments } \\
\text { hypoglycemic events, } \\
\text { laboratory tests, } \\
\text { electrocardiographic and physical examinations, and vital signs } \\
\text { genital infection } \\
\text { urinary tract infection (UTI) } \\
\text { renal impairment, renal failure, kidney stones, } \\
\text { hypotension/dehydration/hypovolemia } \\
\text { change from baseline in total BMD (grams per square centimeter) }\end{array}$ \\
\hline Inclusion criteria & $\begin{array}{l}\text { men and women, } \\
\text { T2DM, } \\
\text { women aged } 55-75 \text { years who were postmenopausal for a period } \\
\text { of at least } 5 \text { years (or to have had an oophorectomy) for at least } 5 \\
\text { years prior,) or men aged } 30-75 \text { years } \\
\text { hemoglobin A1c (HbA1c) } 6.5-8.5 \% \text {, }\end{array}$ \\
\hline
\end{tabular}




\begin{tabular}{|c|c|}
\hline & $\begin{array}{l}\text { fasting plasma glucose (FPG) less than or equal to } 240 \mathrm{mg} / \mathrm{dl}(\leq 13.2 \\
\mathrm{mmol} / \mathrm{l}) \text {; body mass index (BMI) of } 25 \mathrm{~kg} / \mathrm{m} 2 \text { or higher, } \\
\text { body weight no higher than } 120 \mathrm{~kg} \text { (due to limitations imposed by } \\
\text { DXA equipment), } \\
\text { treatment exclusively with metformin at a stable dose of at least } \\
1500 \mathrm{mg} / \mathrm{d} \text { for at least } 12 \text { weeks before enrolment. }\end{array}$ \\
\hline Exclusion criteria & $\begin{array}{l}\text { Exclusion criteria } \\
\text { men under } 30 \text { years and perimenopausal women } \\
\text { poor glycemic control (HbA1c } 8.5 \%) \\
\text { BMD T-scores less than -2.0 at lumbar spine, femoral neck or total } \\
\text { hip regions at the baseline DXA measurement } \\
\text { receiving treatments known to significantly influence bone } \\
\text { metabolism (e.g. bisphosphonates, calcitonin, corticosteroids or } \\
\text { hormone replacement therapy) were excluded. }\end{array}$ \\
\hline \multirow[t]{2}{*}{ Notes } & ClinicalTrials.gov identifier: NCT00855166 \\
\hline & Bolinder 2012 is 78-week extension of Bolinder 2012. \\
\hline
\end{tabular}

\section{Risk of bias table}

\begin{tabular}{|l||l|l||}
\hline Bias & $\begin{array}{l}\text { Authors' } \\
\text { judgement }\end{array}$ & Support for judgement \\
\hline $\begin{array}{l}\text { Random sequence } \\
\text { generation } \\
\text { (selection bias) }\end{array}$ & Low risk & $\begin{array}{l}\text { Patients were allocated to study treatments according to } \\
\text { a predefined computer-generated randomization scheme } \\
\text { provided by AstraZeneca. Balanced block sizes of four to } \\
\text { ensure approximately equal numbers of patients across } \\
\text { the treatment groups and within each stratum (in two } \\
\text { strata, men and women). }\end{array}$ \\
\hline $\begin{array}{l}\text { Allocation } \\
\text { concealment } \\
\text { (selection bias) }\end{array}$ & $\begin{array}{l}\text { Low risk } \\
\text { Bristol-Myers Squibb had access to the randomization } \\
\text { codes during the 24-wk double-blind treatment period. }\end{array}$ \\
\hline $\begin{array}{l}\text { Blinding of } \\
\text { participants and } \\
\text { personnel } \\
\text { (performance bias) }\end{array}$ & Low risk & $\begin{array}{l}\text { Patients and investigators were blinded to study } \\
\text { treatment. All investigational products (dapagliflozin 10 } \\
\text { mg and matching placebo) were identical in appearance, }\end{array}$ \\
\hline $\begin{array}{l}\text { Blinding of } \\
\text { outcome } \\
\text { assessment }\end{array}$ & Low risk & $\begin{array}{l}\text { No patients, investigators, or personnel at AstraZeneca or } \\
\text { Bristol-Myers Squibb had access to the randomization } \\
\text { codes during the 24-wk double-blind treatment period. }\end{array}$ \\
\hline
\end{tabular}




\begin{tabular}{|c|c|c|}
\hline (detection bias) & & \\
\hline $\begin{array}{l}\text { Incomplete } \\
\text { outcome data } \\
\text { (attrition bias) }\end{array}$ & Low risk & $\begin{array}{l}\text { 169/182 completed. } \\
\text { Two analysis sets were defined: the safety analysis set, } \\
\text { consisting of all patients who received at least one dose of } \\
\text { investigational product, and the full analysis set, } \\
\text { consisting of all randomized patients who received at } \\
\text { least one dose of investigational product and who had } \\
\text { both a baseline and at least one post-baseline efficacy } \\
\text { value for at least one efficacy variable. } \\
\text { Primary, key secondary, and exploratory endpoints were } \\
\text { analyzed using the full analysis set. } \\
\text { For glycemic variables, observations after initiation of } \\
\text { rescue therapy were excluded from the analysis, with } \\
\text { these and other missing values for glycemic and non- } \\
\text { glycemic variables at week } 24 \text { replaced using the last } \\
\text { observation carried forward (LOCF) method. } \\
\text { All drop outs and losses to follow up accounted for. }\end{array}$ \\
\hline $\begin{array}{l}\text { Selective reporting } \\
\text { (reporting bias) }\end{array}$ & Low risk & $\begin{array}{l}\text { Besides the major prespecified outcomes, a number of } \\
\text { exploratory endpoints were assessed (but these were } \\
\text { clinically relevant). }\end{array}$ \\
\hline Other bias & Unclear risk & $\begin{array}{l}\text { This study was sponsored by AstraZeneca and Bristol- } \\
\text { Myers Squibb. Some of the trialists are employees of } \\
\text { Bristol-Myers Squibb. }\end{array}$ \\
\hline
\end{tabular}

Cefalu 2013

\begin{tabular}{|c|c|}
\hline Methods & $\begin{array}{l}\text { 52-week, randomized, double-blind, active-controlled, phase } 3 \text { non- } \\
\text { inferiority trial at } 157 \text { centers in } 19 \text { countries with double-blind } \\
\text { extension to } 104 \text { weeks }\end{array}$ \\
\hline Participants & $\begin{array}{l}1452 \text { initially randomized: canagliflozin } 100 m g ~ n=483 \text {; canagliflozin } \\
300 \mathrm{mg} \mathrm{n=485;} \mathrm{glimepiride} n=482 \text {. }\end{array}$ \\
\hline Interventions & $\begin{array}{l}\text { Canagliflozin } 100 \mathrm{mg} \text { or } 300 \mathrm{mg} \text {, versus glimepiride (up-titrated to } 6 \\
\text { mg or } 8 \mathrm{mg} \text { per day), (1:1:1) orally once daily for } 104 \text { weeks. } \\
\text { Add on: insulin, metformin }\end{array}$ \\
\hline Outcomes & Outcomes assessed at week 104. \\
\hline
\end{tabular}




\begin{tabular}{|c|c|}
\hline & $\begin{array}{l}\text { Primary outcomes of the trial } \\
\text { change in HbA1c from baseline to week } 52 \text { and week } 104 \text {, with a } \\
\text { non-inferiority margin of } 0 \cdot 3 \% \text { for the comparison of each } \\
\text { canagliflozin dose with glimepiride. } \\
\text { Secondary outcomes of the trial } \\
\text { proportion of participants achieving HbA1c }<7 \cdot 0 \% \text { or } 6 \cdot 5 \% \text {, } \\
\text { change in fasting plasma glucose } \\
\text { systolic and diastolic blood pressure } \\
\text { percentage change in fasting plasma lipids, including HDL } \\
\text { cholesterol, triglycerides, LDL cholesterol, non-HDL cholesterol, } \\
\text { and ratio of LDL cholesterol to HDL cholesterol. } \\
\text { Adverse events and safety assessments } \\
\text { safety with adverse event reports, } \\
\text { laboratory tests, } \\
\text { vital sign measurements, physical examinations, } \\
\text { self-monitored blood glucose, } \\
12 \text {-lead electro cardiograms, } \\
\text { genital mycotic infections, } \\
\text { urinary tract infections. }\end{array}$ \\
\hline Inclusion criteria & $\begin{array}{l}\text { men and women with T2DM, } \\
\text { aged } 18 \text { to } 80 \text { years } \\
\text { with glycated hemoglobin A1c (HbA1c) of } 7 \cdot 0-9 \cdot 5 \% \text { on stable } \\
\text { metformin } \\
\text { receiving stable metformin therapy ( } \geq 2000 \mathrm{mg} \text { per day or } \geq 1500 \\
\text { mg per day if unable to tolerate a higher dose) for at least } 10 \\
\text { weeks. }\end{array}$ \\
\hline Exclusion criteria & $\begin{array}{l}\text { a history of more than one severe hypoglycemic episode (within } 6 \\
\text { months) } \\
\text { repeated measurements of fasting plasma glucose or fasting self- } \\
\text { monitored blood glucose, or both, of } 15.0 \mathrm{mmol} / \mathrm{L} \text { or more during } \\
\text { the pretreatment phase, } \\
\mathrm{EGFR}<55 \mathrm{~mL} / \mathrm{min} / 1 \cdot 73 \mathrm{~m}^{2} \text { (or }<60 \mathrm{~mL} / \mathrm{min} / 1 \cdot 73 \mathrm{~m}^{2} \text { if based on } \\
\text { restriction of metformin use in local label), } \\
\text { serum creatinine concentrations of } 124 \mu \mathrm{mol} / \mathrm{L} \text { or more for men } \\
\text { and } 115 \mu \mathrm{mol} / \mathrm{L} \text { or more for women, } \\
\text { on thiazolidinedione within } 16 \text { weeks before screening. }\end{array}$ \\
\hline Notes & ClinicalTrials.gov identifier: NCT00968812 \\
\hline
\end{tabular}


The study consisted of a 2 week, single-blind, placebo run-in period and a 52 week, double-blind, core treatment period, followed by a 52 week, double-blind, extension period included in our analyses (Leiter 2015).

\section{Risk of bias table}

\begin{tabular}{|c|c|c|}
\hline Bias & $\begin{array}{l}\text { Authors' } \\
\text { judgement }\end{array}$ & Support for judgement \\
\hline $\begin{array}{l}\text { Random sequence } \\
\text { generation } \\
\text { (selection bias) }\end{array}$ & Low risk & $\begin{array}{l}\text { Participants were then randomly assigned, in a 1:1:1 ratio, } \\
\text { by an interactive voice or web response system. The } \\
\text { sponsor prepared the computer-generated randomization } \\
\text { schedule before the study. Randomization was balanced } \\
\text { with the use of permuted blocks of three patients per } \\
\text { block and stratified by whether the patient was taking a } \\
\text { stable, protocol specified dose of metformin before } \\
\text { screening versus whether they had either undergone } \\
\text { metformin dose adjustment or discontinued use of a } \\
\text { second anti-hyperglycemic drug, or both, and by country. }\end{array}$ \\
\hline $\begin{array}{l}\text { Allocation } \\
\text { concealment } \\
\text { (selection bias) }\end{array}$ & Unclear risk & $\begin{array}{l}\text { Not described but randomization was performed using an } \\
\text { interactive voice response system. }\end{array}$ \\
\hline $\begin{array}{l}\text { Blinding of } \\
\text { participants and } \\
\text { personnel } \\
\text { (performance bias) }\end{array}$ & Low risk & $\begin{array}{l}\text { Double blind placebo. After randomization, } \mathrm{HbA} 1 \mathrm{c} \text { and } \\
\text { fasting plasma glucose values were masked to staff at the } \\
\text { study centers unless values met glycemic rescue criteria } \\
\text { (and were subsequently provided unmasked). Patients, } \\
\text { study investigators, and local sponsor personnel were } \\
\text { masked to treatment assignment until final database lock. } \\
\text { To maintain masked treatment, study drug was supplied } \\
\text { in levels (levels one to five) to allow for masked increases } \\
\text { and decreases of glimepiride throughout the double-blind } \\
\text { treatment period. }\end{array}$ \\
\hline $\begin{array}{l}\text { Blinding of } \\
\text { outcome } \\
\text { assessment } \\
\text { (detection bias) }\end{array}$ & Low risk & $\begin{array}{l}\text { Double blind placebo. HbA1c and fasting plasma glucose } \\
\text { values were masked to staff at the study centers unless } \\
\text { values met glycemic rescue criteria (and were } \\
\text { subsequently provided unmasked). Patients, study } \\
\text { investigators, and local sponsor personnel were masked } \\
\text { to treatment assignment until final database lock. }\end{array}$ \\
\hline Incomplete & Low risk & $1161 / 1452$ completed. Drop outs and losses to follow up \\
\hline
\end{tabular}




\begin{tabular}{|l||l|l||}
\hline $\begin{array}{l}\text { outcome data } \\
\text { (attrition bias) }\end{array}$ & $\begin{array}{l}\text { accounted for. Analyses performed using intention to } \\
\text { treat carried forward. }\end{array}$ \\
\hline $\begin{array}{l}\text { Selective reporting } \\
\text { (reporting bias) }\end{array}$ & Low risk & All clinically relevant outcomes are defined and reported. \\
\hline Other bias & Unclear risk & $\begin{array}{l}\text { The sponsor of the study had a role in study design and } \\
\text { conduct; data collection, analysis, and interpretation; and } \\
\text { writing of the article. }\end{array}$ \\
\hline
\end{tabular}

\section{Cefalu 2015}

\begin{tabular}{|c|c|}
\hline Methods & $\begin{array}{l}\text { 52-week, multicenter, randomized, parallel-group, double-blind, } \\
\text { multicenter, placebo-controlled study in } 5 \text { counties/continents } \\
\text { (Europe, Asia, USA, Canada, and Argentina). }\end{array}$ \\
\hline Participants & $\begin{array}{l}922 \text { initially randomized (to main assessment, to sub study): } \\
\text { dapagliflozin } 10 \mathrm{mg} n=455 \text {; placebo } n=459 \text {. }\end{array}$ \\
\hline Interventions & $\begin{array}{l}\text { Patients were randomized in a 1:1 ratio to double-blind treatment } \\
\text { with either dapagliflozin } 10 \mathrm{mg} \text { or placebo for } 24 \text { weeks extended to } \\
52 \text { weeks. } \\
\text { Add on: pre-existing stable background treatment (e.g. insulin, } \\
\text { metformin) other than rosiglitazone. } \\
\text { Rescue therapy permitted. }\end{array}$ \\
\hline Outcomes & $\begin{array}{l}\text { At } 52 \text { weeks: } \\
\text { Primary outcomes of the trial } \\
\text { mean change in HbA1c from baseline to week } 24 \\
\text { the proportion of responders achieving a three-item end point of } \\
\text { combined clinical benefit at week } 24 \text { (an absolute drop from } \\
\text { baseline in HbA1c of } \geq 0.5 \% \text { ( } 5.5 \mathrm{mmol} / \mathrm{mol} \text { ), a relative drop of } \geq 3 \% \\
\text { for total BW, and an absolute drop of } \geq 3 \mathrm{mmHg} \text { from baseline in } \\
\text { seated SBP. } \\
\text { Secondary outcomes of the trial } \\
\text { mean change in seated SBP from baseline (at weeks } 8 \text { and } 24 \text { ), } \\
\text { mean percent body weight } \\
\text { mean change in body weight from baseline; } \\
\text { proportion with baseline BMI of } \geq 27 \mathrm{~kg} / \mathrm{m} 2 \text { with a } \geq 5 \% \text { reduction in } \\
\text { BW. } \\
\text { mean change in seated diastolic blood pressure (DBP); }\end{array}$ \\
\hline
\end{tabular}




\begin{tabular}{|c|c|}
\hline & $\begin{array}{l}\text { proportion with seated SBP of }<130 \mathrm{mmHg} \text { in the group of patients } \\
\text { with a baseline seated SBP of } \geq 130 \mathrm{mmHg} \text {; } \\
\text { mean change in HbA1c in patients with a baseline } \mathrm{HbA} 1 \mathrm{c} \text { mean } \\
\text { change in body weight from baseline } \geq 8.0 \% \text { ( } 64 \mathrm{mmol} / \mathrm{mol} \text { ) and an } \\
\mathrm{HbA} 1 \mathrm{c} \geq 9.0 \% \text { ( } 75 \mathrm{mmol} / \mathrm{mol}) ; \\
\text { proportion achieving an } \mathrm{HbA} 1 \mathrm{c}<7.0 \% \text { ( } 53 \mathrm{mmol} / \mathrm{mol}) \text {; } \\
\text { mean change in FPG at weeks } 1 \text { and } 24 ; \\
\text { proportion rescued for failing to maintain FPG/HbA1c below the } \\
\text { prespecified rescue criteria at weeks } 4,8,16,24 \text { and } 52 ; \\
\text { proportion of patients achieving a reduction in HbA1c of } \geq 0.5 \% \text { ( } 5.5 \\
\text { mmol/mol); } \\
\text { proportion achieving a reduction in seated SBP from baseline of } \geq 3 \\
\text { or } \geq 5 \text { mmHg; } \\
\text { mean change in calculated average daily insulin dose in patients } \\
\text { treated with insulin at baseline. } \\
\text { Adverse events and safety assessments (all patients who received } \\
\text { one or more doses of randomized study medication and who } \\
\text { provided safety records over the } 52 \text { weeks) } \\
\text { all safety and tolerability events including: } \\
\text { CV events, } \\
\text { laboratory values, } \\
\text { electrocardiogram results, } \\
\text { vital signs, } \\
\text { hypoglycemic events, } \\
\text { calculated creatinine clearance, } \\
\text { estimated glomerular filtration rate (eGFR), } \\
\text { physical examination findings. }\end{array}$ \\
\hline Inclusion criteria & $\begin{array}{l}\text { Men aged } \geq 45 \text { years or women } \geq 50 \text { years (not of childbearing } \\
\text { potential) with type } 2 \text { diabetes, } \\
\text { on monotherapy or dual combination therapy with oral } \\
\text { antidiabetic drugs (OADs), insulin therapy in combination with } \\
\text { OADs, or insulin monotherapy on a daily basis for } 8 \text { weeks, were } \\
\text { stable for at least } 4 \text { weeks before enrolment, and showed } \\
\text { inadequate glycemic control ( } 7.2 \% \text { ( } 55 \mathrm{mmol} / \mathrm{mol} \text { ) } \leq \mathrm{HbA} 1 \mathrm{c} \leq 10.5 \% \\
\text { ( } 91 \mathrm{mmol} / \mathrm{mol}) \text { ). } \\
\text { cerebrovascular disease, and hypertension eligible if treatment } \\
\text { uninterruptedly on a daily basis in the last } 4 \text { weeks before } \\
\text { enrolment. } \\
\text { hypertension was defined as prior physician-made diagnosis of } \\
\text { essential hypertension, i.e., before screening, or treatment with } \\
\text { two or more antihypertensive agents (diuretics, } \beta \text {-blockers, } \\
\text { angiotensin-converting enzyme inhibitors, angiotensin receptor } \\
\text { blockers, or calcium channel antagonists), with one of the agents }\end{array}$ \\
\hline
\end{tabular}




\begin{tabular}{|c|c|}
\hline & $\begin{array}{l}\text { started for lowering blood pressure (BP), or treatment with one } \\
\text { antihypertensive agent and a past physician recording of a BP } \\
\text { exceeding } 130 / 80 \mathrm{mmHg} \text {. } \\
\text { cardiovascular (CV) disease was defined as coronary heart disease, } \\
\text { coronary artery stenosis }>50 \% \text {, stroke or transient ischemic attack, } \\
\text { or peripheral artery disease treated with revascularization } \\
\text { (amputation was not accepted). }\end{array}$ \\
\hline Exclusion criteria & $\begin{array}{l}\text { Exclusion criteria } \\
\text { type } 1 \text { diabetes mellitus, } \\
\text { more than three oral antidiabetic medications, } \\
\text { fasting plasma glucose }(F P G)>15 \mathrm{mmol} / \mathrm{l} \text { at randomization, } \\
\text { diabetic ketoacidosis, } \\
\text { recent CV event, } \\
\text { systolic BP (SBP) } \geq 165 \mathrm{mmHg} \text {, diastolic BP (DBP) } \geq 100 \mathrm{mmHg} \text {, } \\
\text { congestive heart failure (CHF) } \\
\text { calculated creatinine clearance }<60 \mathrm{ml} / \mathrm{min} \text {, } \\
\text { severe hepatic insufficiency and/or significant abnormal liver } \\
\text { function. }\end{array}$ \\
\hline Notes & $\begin{array}{l}\text { 24-week trial with } 28 \text {-week extension, therefore } 52 \text {-week outcome } \\
\text { data are used in our analyses (a 52-week long extension study is } \\
\text { ongoing). } \\
\text { ClinicalTrials.gov identifier NСТ01031680 }\end{array}$ \\
\hline
\end{tabular}

Risk of bias table

\begin{tabular}{|l|l||l||}
\hline Bias & $\begin{array}{l}\text { Authors' } \\
\text { judgement }\end{array}$ & Support for judgement \\
\hline $\begin{array}{l}\text { Random sequence } \\
\text { generation } \\
\text { (selection bias) }\end{array}$ & Low risk & $\begin{array}{l}\text { Participants were assigned a unique enrolment number } \\
\text { using Interactive Web Response System (IWRS) or } \\
\text { Interactive Voice Response System (IVRS) at visit 1 } \\
\text { (Enrollment Visit). Participants were randomized to the } \\
\text { treatment groups using the method of randomly } \\
\text { permuted blocks with a block size of 4 after evaluation for } \\
\text { all inclusion/exclusion criteria. Participants were stratified } \\
\text { into one of the eight age-by-insulin use-by-time from most } \\
\text { recent qualifying CV event strata } \\
\text { according to age group (<65 years vs. } \geq 65 \text { years at } \\
\text { enrolment), use of insulin (No versus Yes at } \\
\text { randomization), and time from most recent qualifying CV }\end{array}$ \\
\hline
\end{tabular}




\begin{tabular}{|c|c|c|}
\hline & & $\begin{array}{l}\text { event (more than } 1 \text { year vs. } 1 \text { year } \\
\text { or less [i.e., within } 12 \text { months] before enrolment). } \\
\text { Participants were given medication with the bottle } \\
\text { number allocated by the IWRS/IVRS. }\end{array}$ \\
\hline $\begin{array}{l}\text { Allocation } \\
\text { concealment } \\
\text { (selection bias) }\end{array}$ & Low risk & $\begin{array}{l}\text { Centralized randomization system used. Participants were } \\
\text { given medication with the bottle number allocated by the } \\
\text { IWRS/IVRS. }\end{array}$ \\
\hline $\begin{array}{l}\text { Blinding of } \\
\text { participants and } \\
\text { personnel } \\
\text { (performance bias) }\end{array}$ & Low risk & Double blind placebo. \\
\hline $\begin{array}{l}\text { Blinding of } \\
\text { outcome } \\
\text { assessment } \\
\text { (detection bias) }\end{array}$ & Low risk & Double blind placebo. \\
\hline $\begin{array}{l}\text { Incomplete } \\
\text { outcome data } \\
\text { (attrition bias) }\end{array}$ & Low risk & $\begin{array}{l}807 / 922 \text { completed. All drop outs and losses to follow up } \\
\text { accounted for. } \\
\text { The last observation carried forward approach was used } \\
\text { for all variables at } 24 \text { weeks. ITT analysis employed. }\end{array}$ \\
\hline $\begin{array}{l}\text { Selective reporting } \\
\text { (reporting bias) }\end{array}$ & Low risk & $\begin{array}{l}\text { All clinically relevant outcomes were defined and } \\
\text { reported. }\end{array}$ \\
\hline Other bias & Unclear risk & $\begin{array}{l}\text { This study was sponsored by Bristol-Myers Squibb and } \\
\text { AstraZeneca. Medical writing assistance was funded by } \\
\text { AstraZeneca. Some of the investigators are employees of } \\
\text { Astra Zeneca. }\end{array}$ \\
\hline
\end{tabular}

\section{DeFronzo 2015}

\begin{tabular}{||l|l||}
\hline Methods & $\begin{array}{l}52 \text { week, phase 3, randomized, double blind, parallel-group study in } \\
197 \text { centers in } 22 \text { countries. }\end{array}$ \\
\hline Participants & $\begin{array}{l}686 \text { initially randomized, empagliflozin } 25 \mathrm{mg} / \text { linagliptin } 5 \mathrm{mg}(\mathrm{n}= \\
137) ; \text { empagliflozin } 10 \mathrm{mg} / \text { linagliptin } 5 \mathrm{mg}(\mathrm{n}=136) \text {; empagliflozin } 25 \\
\mathrm{mg}(\mathrm{n}=141) ; \text { empagliflozin } 10 \mathrm{mg}(\mathrm{n}=140) \text {; or linagliptin } 5 \mathrm{mg}(\mathrm{n}= \\
132) \text { as add-on to metformin. }\end{array}$ \\
\hline Interventions & $\begin{array}{l}\text { Randomized (1:1:1:1:1) to receive empagliflozin } 25 \mathrm{mg} / \text { linagliptin } 5 \\
\mathrm{mg}, \text { empagliflozin } 10 \mathrm{mg} / \text { linagliptin } 5 \mathrm{mg} \text { FDC tablet, empagliflozin }\end{array}$ \\
\hline
\end{tabular}




\begin{tabular}{|c|c|}
\hline & $\begin{array}{l}25 \mathrm{mg} \text {, empagliflozin } 10 \mathrm{mg} \text {, or linagliptin } 5 \mathrm{mg} \text { for } 52 \text { weeks. } \\
\text { Add on: metformin. } \\
\text { Rescue initiated if a subject had blood glucose }>240 \mathrm{mg} / \mathrm{dL} \text {. }\end{array}$ \\
\hline Outcomes & 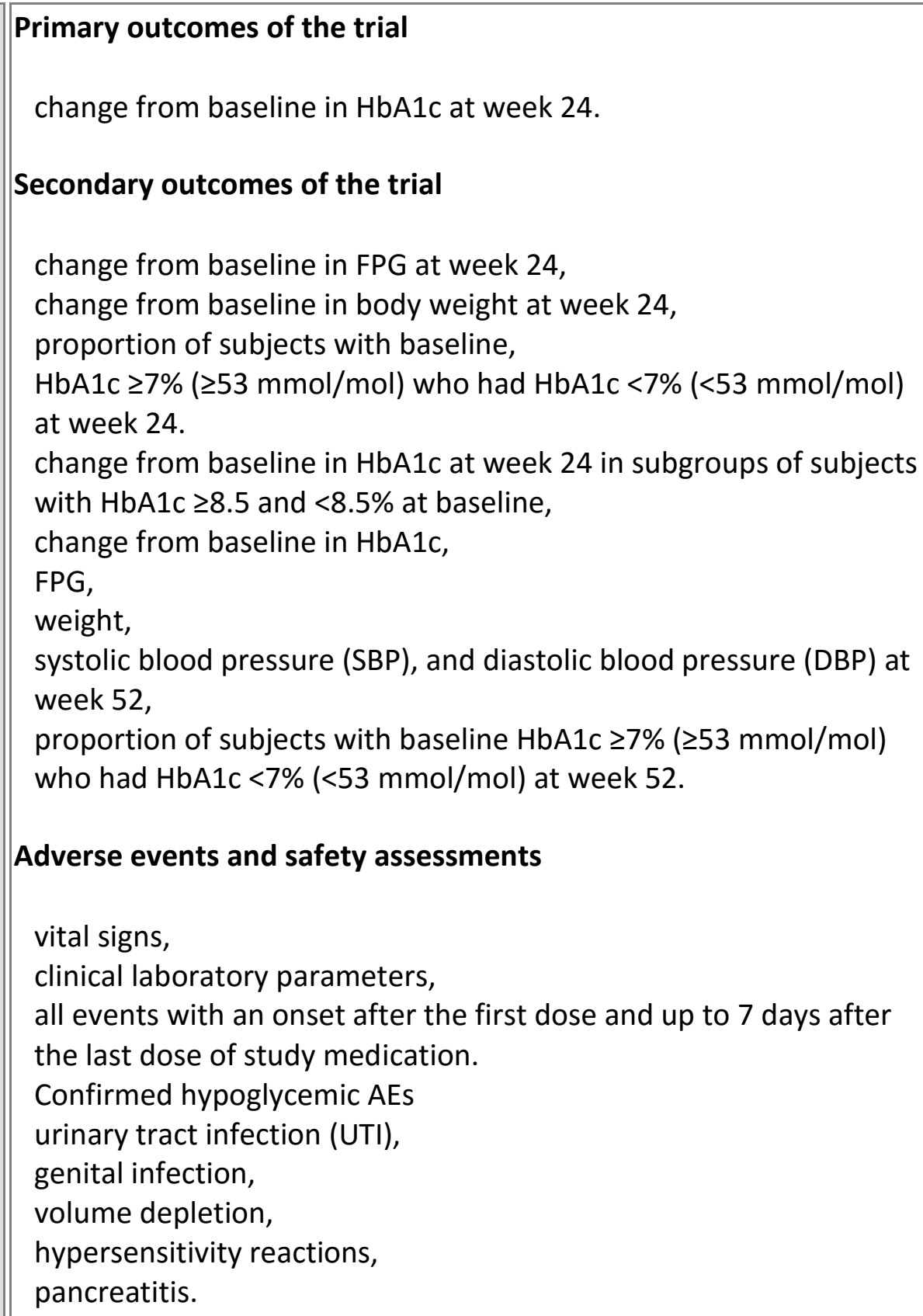 \\
\hline Inclusion criteria & $\begin{array}{l}\text { men and women } \\
\text { aged }>18 \text { years } \\
\mathrm{BMI}>45 \mathrm{~kg} / \mathrm{m}^{2} \\
\mathrm{HbA} 1 \mathrm{c}>7 \text { to } \leq 10.5 \% \text { ( }>53 \text { to } \leq 91 \mathrm{mmol} / \mathrm{mol} \text { ) at screening } \\
\text { with metformin immediate release }(\geq 1,500 \mathrm{mg} / \text { day, maximum } \\
\text { tolerated dose, or maximum dose according to local label) at an } \\
\text { unchanged dose for } \geq 12 \text { weeks prior to randomization }\end{array}$ \\
\hline
\end{tabular}




\begin{tabular}{||l||l||}
\hline \hline Exclusion criteria & $\begin{array}{l}\text { on a diet and exercise regimen. } \\
\text { uncontrolled hyperglycemia (glucose level }>240 \mathrm{mg} / \mathrm{dL} \text { after an } \\
\text { overnight fast, } \\
\text { treatment with any antidiabetes drug except metformin within } 12 \\
\text { weeks prior to randomization } \\
\text { eGFR }<60 \mathrm{~mL} / \mathrm{min} / 1.73 \mathrm{~m} 2 \text { using the Modification of Diet in Renal } \\
\text { Disease (MDRD) equation, } \\
\text { acute coronary syndrome, } \\
\text { stroke, or transient ischemic attack within } 3 \text { months prior to } \\
\text { consent, } \\
\text { bariatric surgery in the last } 2 \text { years, } \\
\text { investigational drug intake within } 1 \text { month prior to consent, } \\
\text { treatment with anti-obesity drugs within } 3 \text { months prior to } \\
\text { consent. }\end{array}$ \\
\hline Notes & $\begin{array}{l}\text { ClinicalTrials.gov identifier: NCT01422876 } \\
\text { DeFronzo } 2015 \text { participants randomized to receive empagliflozin for }\end{array}$ \\
\hline $\begin{array}{ll}\text { 24 weeks, and this was extended to } 52 \text { weeks within the trial, } \\
\text { however the primary endpoint of change in HbA1c was measured at } \\
24 \text { weeks. The } 52 \text {-week data are included in our analyses. }\end{array}$ \\
\hline \hline
\end{tabular}

\section{Risk of bias table}

\begin{tabular}{||l||l|l||}
\hline Bias & $\begin{array}{l}\text { Authors' } \\
\text { judgement }\end{array}$ & Support for judgement \\
\hline $\begin{array}{l}\text { Random sequence } \\
\text { generation (selection } \\
\text { bias) }\end{array}$ & Low risk & $\begin{array}{l}\text { Interactive voice and web response system. } \\
\text { "Randomization was performed using a third-party } \\
\text { interactive voice and web response system" (stratified } \\
\text { by HbA1c at screening and region (Europe, Asia, North } \\
\text { America, and South America). }\end{array}$ \\
\hline $\begin{array}{l}\text { Allocation } \\
\text { concealment } \\
\text { (selection bias) }\end{array}$ & Unclear risk & $\begin{array}{l}\text { Not described in detail but implied in computer } \\
\text { generated random system. }\end{array}$ \\
\hline $\begin{array}{l}\text { Blinding of } \\
\text { participants and } \\
\text { personnel } \\
\text { (performance bias) }\end{array}$ & Low risk & Double blind. \\
\hline $\begin{array}{l}\text { Blinding of outcome } \\
\text { assessment }\end{array}$ & Low risk & Double blind. \\
\hline
\end{tabular}




\begin{tabular}{|l||l||l||}
\hline (detection bias) & & \\
\hline $\begin{array}{l}\text { Incomplete outcome } \\
\text { data (attrition bias) }\end{array}$ & Low risk & $\begin{array}{l}\text { 674/686 completed (full analysis set; FAS). A last } \\
\text { observation carried forward (LOCF) approach was used } \\
\text { to impute missing continuous efficacy data. }\end{array}$ \\
\hline \begin{tabular}{l|l|l} 
Selective reporting \\
(reporting bias)
\end{tabular} & Low risk & $\begin{array}{l}\text { All clinically relevant outcomes are defined and } \\
\text { reported. }\end{array}$ \\
\hline Other bias & Unclear risk & $\begin{array}{l}\text { Medical writing assistance, supported financially by } \\
\text { Boehringer Ingelheim. This study was funded by } \\
\text { Boehringer Ingelheim and Eli Lilly and Company. }\end{array}$ \\
\hline \hline
\end{tabular}

\section{Ferrannini 2010}

\begin{tabular}{|c|c|}
\hline Methods & $\begin{array}{l}\text { 24-week randomized parallel-group, double-blind, placebo- } \\
\text { controlled phase } 3 \text { trial at } 85 \text { sites in the U.S., Canada, Mexico, and } \\
\text { Russia. }\end{array}$ \\
\hline Participants & $\begin{array}{l}485 \text { initially randomized, } 274 \text { joined main cohort: } 2.5 \mathrm{mg} \mathrm{n}=65 ; 5 \mathrm{mg} \\
n=64 \text {; or } 10 \mathrm{mg} n=70 \text {; dapagliflozin or placebo } n=75 \text {. }\end{array}$ \\
\hline Interventions & $\begin{array}{l}\text { Randomly assigned equally once-daily placebo or } 2.5,5 \text {, or } 10 \mathrm{mg} \\
\text { dapagliflozin, administered once daily either in the morning for } 24 \\
\text { weeks. } \\
\text { Rescue medication permitted. } \\
\text { Add on: diet/exercise counselling given. }\end{array}$ \\
\hline Outcomes & $\begin{array}{l}\text { Primary outcomes of the trial } \\
\text { change from baseline in A1C at week } 24 \\
\text { Secondary outcomes of the trial } \\
\text { change from baseline at week } 24 \text { in FPG } \\
\text { body weight. } \\
\text { Adverse events and safety assessments } \\
\text { vital signs, laboratory measurements, } \\
\text { and adverse events } \\
\text { urinary tract infections (UTIs) } \\
\text { genital infections } \\
\text { self-monitored blood glucose daily for unusually high or low blood }\end{array}$ \\
\hline
\end{tabular}




\begin{tabular}{|c|c|}
\hline & glucose event or any symptoms suggestive of hypoglycemia. \\
\hline Inclusion criteria & $\begin{array}{l}\text { men and women } \\
\text { T2DM } \\
\text { aged } 18-77 \text { years } \\
\text { BMI } \leq 45 \mathrm{~kg} / \mathrm{m}^{2} \\
\text { fasting Cpeptide } \geq 1.0 \mathrm{ng} / \mathrm{ml} \\
\text { A1C } 7.0-10 \% \\
\text { treatment naive }\end{array}$ \\
\hline Exclusion criteria & $\begin{array}{l}\text { history of type } 1 \\
\text { diabetes, serum creatinine } \geq 133 \mu \mathrm{mol} / \mathrm{l} \text { (men) or } \geq 124 \mu \mathrm{mol} / \mathrm{l} \\
\text { (women), } \\
\text { urine albumin-to-creatinine ratio }>200 \mathrm{mg} / \mathrm{mmol} \text {, } \\
\text { aspartate transaminase and/or alanine transaminase }>3 \text { times the } \\
\text { upper limits of normal, } \\
\text { creatine kinase } \geq 3 \text { times the upper limit of normal, } \\
\text { symptoms of severely uncontrolled diabetes (including marked } \\
\text { polyuria and polydipsia with }>10 \% \text { weight loss during the last } 3 \\
\text { months before enrolment) } \\
\text { significant disease: renal hepatic, hematological, oncological, } \\
\text { endocrine, psychiatric, rheumatic diseases, } \\
\text { a cardiovascular event (including New York Heart Association class } \\
\text { III/IV congestive heart failure) within } 6 \text { months of enrolment, } \\
\text { severe uncontrolled blood pressure (systolic blood pressure } \geq 180 \\
\text { mmHg and/or diastolic blood pressure } \geq 110 \mathrm{mmHg} \text { ). }\end{array}$ \\
\hline Notes & $\begin{array}{l}\text { Dapagliflozin } 10 \text { mg given in the morning vs placebo. Participants } \\
\text { with high A1c put on open label rescue medicine - not included in } \\
\text { our analyses. Results from main cohort only used in our analyses. } \\
\text { ClinicalTrials.gov identifier: NCT00528372 }\end{array}$ \\
\hline
\end{tabular}

Risk of bias table

\begin{tabular}{||l|l|l||}
\hline Bias & $\begin{array}{l}\text { Authors' } \\
\text { judgement }\end{array}$ & Support for judgement \\
\hline $\begin{array}{l}\text { Random sequence } \\
\text { generation (selection bias) }\end{array}$ & Unclear risk & Randomly assigned. \\
\hline $\begin{array}{l}\text { Allocation concealment } \\
\text { (selection bias) }\end{array}$ & Unclear risk & Not mentioned. \\
\hline Blinding of participants & Low risk & Double blind placebo. \\
\hline
\end{tabular}




\begin{tabular}{|l|l||l||}
\hline $\begin{array}{l}\text { and personnel } \\
\text { (performance bias) }\end{array}$ & & \\
\hline $\begin{array}{l}\text { Blinding of outcome } \\
\text { assessment (detection } \\
\text { bias) }\end{array}$ & Low risk & Double blind placebo. \\
\hline $\begin{array}{l}\text { Incomplete outcome data } \\
\text { (attrition bias) }\end{array}$ & Low risk & $\begin{array}{l}232 / 274 \text { completed. All drop outs and losses to } \\
\text { follow up accounted for. }\end{array}$ \\
\hline $\begin{array}{l}\text { Selective reporting } \\
\text { (reporting bias) }\end{array}$ & Low risk & $\begin{array}{l}\text { All clinical relevant data are defined and } \\
\text { reported. }\end{array}$ \\
\hline Other bias & Unclear risk & $\begin{array}{l}\text { This study was funded by Bristol-Myers Squibb } \\
\text { and AstraZeneca. A Bristol-Myers Squibb, } \\
\text { employee provided writing and editorial support. }\end{array}$ \\
\hline
\end{tabular}

\section{Ferrannini 2013}

\begin{tabular}{|c|c|}
\hline Methods & $\begin{array}{l}\text { 12-week Phase IIb, randomized, double-blind, placebo-controlled } \\
\text { trial at } 75 \text { centers in } 13 \text { countries. }\end{array}$ \\
\hline Participants & $\begin{array}{l}408 \text { initially randomized: placebo } n=82 ; 5 m g \text { empagliflozin } n=81 ; 10 \\
\text { mg empagliflozin } n=81 ; 25 \text { mg empagliflozin } n=82 ; \text { open-label } \\
\text { metformin } n=80 \text {. }\end{array}$ \\
\hline Interventions & $\begin{array}{l}\text { Empagliflozin (5mg empagliflozin; } 10 \mathrm{mg} \text { empagliflozin; } 25 \mathrm{mg} \\
\text { empagliflozin; versus placebo oral dose once daily, double-blind. } \\
\text { Add on: none, other than open label metformin arm } 1000 \mathrm{mg} \text { daily } \\
\text { dose of metformin ( } 500 \mathrm{mg} \text { twice daily with meals) for the first } 4 \\
\text { weeks. If fasted plasma glucose values remained }>6.1 \mathrm{mmol} / \mathrm{l} \text {, the } \\
\text { metformin dose was increased to } 1000 \mathrm{mg} \text { twice daily or up to the } \\
\text { maximum tolerated dose. }\end{array}$ \\
\hline Outcomes & $\begin{array}{l}\text { Primary outcomes of the trial } \\
\text { change in HbA1c from baseline to week } 12 \text {. } \\
\text { Secondary outcomes of the trial } \\
\text { change in FPG from baseline to week } 12 \text {, } \\
\text { change in HbA1c from baseline over time, } \\
\text { proportion of patients who achieved an } \mathrm{HbA} 1 \mathrm{c} \leq 7.0 \% \text { after } 12 \\
\text { weeks of treatment, } \\
\text { proportion of patients who achieved an } \mathrm{HbA} 1 \mathrm{c} \text { lowering of } \geq 0.5 \%\end{array}$ \\
\hline
\end{tabular}




\begin{tabular}{|c|c|}
\hline & $\begin{array}{l}\text { after } 12 \text { weeks of treatment, change in body weight from baseline } \\
\text { to week } 12 \text { and PK of empagliflozin. } \\
\text { Adverse events and safety assessments } \\
\text { incidence and intensity of AEs, } \\
\text { withdrawal because of AEs, } \\
\text { clinically relevant new or worsening findings in physical } \\
\text { examination and vital signs (blood pressure and pulse rate) } \\
\text { reported as AEs, } \\
\text { clinically relevant new or worsening findings in 12-lead ECGs } \\
\text { reported as AEs and clinical laboratory assessments. }\end{array}$ \\
\hline Inclusion criteria & $\begin{array}{l}\text { men and women } \\
\text { aged } \geq 18 \text { and } \leq 79 \text { years } \\
\text { T2DM } \\
\text { treatment naive (no antidiabetic medication for } \geq 10 \text { weeks prior to } \\
\text { screening) or on one antidiabetic drug (except thiazolidinediones, } \\
\text { glucagonlike peptide-1 (GLP-1) analogues or insulin) at a stable } \\
\text { dose for } \geq 10 \text { weeks prior to screening. } \\
\text { HbA } 1 \mathrm{c} \geq 6.5 \text { to } \leq 9.0 \% \text { for patients treated with one other } \\
\text { antidiabetic drug, or HbA1c }>7.0 \text { to } \leq 10.0 \% \text { for treatment-naive } \\
\text { patients. At start of placebo run-in, HbA1c was required to be }>7.0 \\
\text { to } \leq 10.0 \% \text {. } \\
\text { BMI } \leq 40 \mathrm{~kg} / \mathrm{m}^{2}\end{array}$ \\
\hline Exclusion criteria & $\begin{array}{l}\text { myocardial infarction, stroke or transient ischemic attack } \\
\leq 6 \text { months prior to informed consent, } \\
\text { impaired hepatic function, renal insufficiency or impaired renal } \\
\text { function defined as calculated creatinine clearance }<0.84 \mathrm{ml} / \mathrm{s} / \mathrm{m} 2 \\
\text { or serum creatinine levels } \geq 132.6 \mu \text { mol/l for men and } \geq 123.8 \mathrm{~mol} / \mathrm{I} \\
\text { for women, } \\
\text { unstable or acute congestive heart failure, } \\
\text { acute or chronic acidosis, } \\
\text { disease of central nervous system, psychiatric disorders or clinically } \\
\text { relevant neurologic disorders, } \\
\text { chronic or clinically relevant acute infections, } \\
\text { current or chronic urogenital tract infection, } \\
\text { dehydration, } \\
\text { history of clinically relevant allergy/hypersensitivity, intolerance to } \\
\text { metformin, } \\
\text { hereditary galactose intolerance, } \\
\text { treatment with thiazolidinediones, GLP-1 analogues or insulin } \\
\leq 3 \text { months prior to informed consent, } \\
\text { treatment with anti-obesity drugs, } \\
\text { current treatment with systemic steroids, }\end{array}$ \\
\hline
\end{tabular}




\begin{tabular}{||l||l||}
\hline \hline & $\begin{array}{l}\text { alcohol abuse, } \\
\text { treatment with an investigational drug } \leq 2 \text { months prior to } \\
\text { informed consent, } \\
\text { pregnancy, breastfeeding, not practising an acceptable method of } \\
\text { birth control (female patients only). }\end{array}$ \\
\hline Notes & $\begin{array}{l}\text { Empagliflozin vs placebo, background treatment one other OAD (- } \\
\text { glitazones, GLP-1analoges or insulin). Open label metformin arm not } \\
\text { included. } \\
\text { A 78-week extension of the study, EMPA Ferrannini 2013b, is an } \\
\text { open trial, placebo switched to active, data not included in these } \\
\text { analyses. } \\
\text { ClinicalTrials.gov Identifier: NCT00789035 }\end{array}$ \\
\hline
\end{tabular}

\section{Risk of bias table}

\begin{tabular}{|c|c|c|}
\hline Bias & $\begin{array}{l}\text { Authors' } \\
\text { judgement }\end{array}$ & Support for judgement \\
\hline $\begin{array}{l}\text { Random sequence } \\
\text { generation } \\
\text { (selection bias) }\end{array}$ & Low risk & $\begin{array}{l}\text { Interactive voice and web response system, stratified by } \\
\text { country and by number of previous antidiabetic } \\
\text { medications. }\end{array}$ \\
\hline $\begin{array}{l}\text { Allocation } \\
\text { concealment } \\
\text { (selection bias) }\end{array}$ & Unclear risk & $\begin{array}{l}\text { Not described in detail but implied in computer } \\
\text { generated random system. }\end{array}$ \\
\hline $\begin{array}{l}\text { Blinding of } \\
\text { participants and } \\
\text { personnel } \\
\text { (performance bias) }\end{array}$ & Low risk & Double blind. \\
\hline $\begin{array}{l}\text { Blinding of outcome } \\
\text { assessment } \\
\text { (detection bias) }\end{array}$ & Low risk & Double blind. \\
\hline $\begin{array}{l}\text { Incomplete } \\
\text { outcome data } \\
\text { (attrition bias) }\end{array}$ & Unclear risk & $\begin{array}{l}\text { 406/408 who were treated were included in the analysis. } \\
\text { Two participants were randomized but not treated; the } \\
\text { treated set consisted of all randomized patients who } \\
\text { received at least one dose of the study drug. No } \\
\text { participant flow chart was provided. Reasons for } \\
\text { dropouts and losses to follow up were not provided. } \\
\text { Comment: insufficient information, therefore judged as }\end{array}$ \\
\hline
\end{tabular}




\begin{tabular}{||l|l|l||}
\hline \hline & & unclear. \\
\hline $\begin{array}{l}\text { Selective reporting } \\
\text { (reporting bias) }\end{array}$ & Low risk & All clinically relevant outcomes are defined and reported. \\
\hline Other bias & Unclear risk & $\begin{array}{l}\text { Medical writing assistance, supported financially by } \\
\text { Boehringer Ingelheim. Some of the trialists were } \\
\text { employees of Boehringer Ingelheim. }\end{array}$ \\
\hline
\end{tabular}

Forst 2014

\begin{tabular}{|c|c|}
\hline Methods & $\begin{array}{l}26 \text { week randomized, double-blind, placebo-controlled phase } 3 \text { trial } \\
\text { conducted at } 74 \text { centers in } 11 \text { countries. }\end{array}$ \\
\hline Participants & $\begin{array}{l}344 \text { initially randomized, canagliflozin } 100 m g ~ n=113 \text {; canagliflozin } \\
300 m g ~ n=114 \text {; placebo } n=115 \text {. }\end{array}$ \\
\hline Interventions & $\begin{array}{l}\text { Canagliflozin } 100 \text { or } 300 \text { mg versus placebo (oral dose) for } 26 \text { weeks. } \\
\text { Add on: standard counselling on diet and exercise, metformin, } \\
\text { pioglitazone }\end{array}$ \\
\hline Outcomes & $\begin{array}{l}\text { Primary outcomes of the trial } \\
\text { efficacy end-point change from baseline in HbA1c at week } 26 . \\
\text { Secondary outcomes of the trial } \\
\text { proportion of patients reaching HbA1c <7.0\%, } \\
\text { change from baseline in FPG, } \\
\text { systolic BP } \\
\text { fasting index of } \beta \text {-cell function, HomeostasisModel Assessment } \\
\text { (HOMA2-\%B), } \\
\text { percent change from baseline in body weight, high-density } \\
\text { lipoprotein cholesterol (HDL-C) and triglycerides. } \\
\text { Adverse events and safety assessments } \\
\text { Adverse event (AE) reports, } \\
\text { safety laboratory tests, } \\
\text { vital sign measurements, } \\
\text { physical examinations, } \\
\text { SMBG and } 12 \text {-lead electrocardiograms. } \\
\text { urinary tract infections (UTIs), } \\
\text { genital mycotic infections, } \\
\text { specific analyses were performed for AEs related to osmotic }\end{array}$ \\
\hline
\end{tabular}




\begin{tabular}{|c|c|}
\hline & $\begin{array}{l}\text { diuresis and volume depletion, } \\
\text { hypoglycemia episodes. }\end{array}$ \\
\hline Inclusion criteria & $\begin{array}{l}\text { men and women } \\
\text { with T2DM } \\
\text { aged } \geq 18 \text { and } \leq 80 \text { years, } \\
\text { inadequate glycemic control (HbA1c } \geq 7.0 \% \text { ( } 53 \mathrm{mmol} / \mathrm{mol} \text { ) to } \\
\leq 10.5 \% \text { ( } 91 \mathrm{mmol} / \mathrm{mol} \text { )) on stable metformin therapy }(2,000 \\
\mathrm{mg} / \text { day (or } 1,500 \mathrm{mg} / \text { day if unable to tolerate higher dose)) for } 8 \\
\text { weeks } \\
\text { fasting plasma glucose (FPG) }<15 \mathrm{mmol} / \mathrm{l} \text { at week } 2 \\
\text { fasting fingerstick glucose } \geq 6.1 \mathrm{mmol} / \mathrm{l} \text { and }<15 \mathrm{mmol} / \mathrm{l} \text { on day } 1 \text {. }\end{array}$ \\
\hline Exclusion criteria & $\begin{array}{l}\text { FPG and/or fasting self-monitored blood glucose (SMBG) } \geq 15.0 \\
\mathrm{mmol} / \mathrm{l}(270 \mathrm{mg} / \mathrm{dl} \text { ) during the pretreatment phase; } \\
\text { history of type } 1 \text { diabetes, cardiovascular disease (including } \\
\text { myocardial infarction, } \\
\text { unstable angina, revascularization procedure or cerebrovascular } \\
\text { accident) within } 3 \text { months prior to screening, } \\
\text { uncontrolled hypertension; } \\
\text { ongoing eating disorder or } 5 \% \text { change in body weight within } 12 \\
\text { weeks; } \\
\text { eGFR }<55 \mathrm{ml} / \mathrm{min} / 1.73 \mathrm{~m}^{2} \text { (or }<60 \mathrm{ml} / \mathrm{min} / 1.73 \mathrm{~m} 2 \text { if based upon } \\
\text { restriction of metformin use in local label) or } \\
\text { serum creatinine } \geq 124 \mu \mathrm{mol} / \mathrm{l} \text { for men and } \geq 115 \mu \mathrm{mol} / \mathrm{I} \text { for women. }\end{array}$ \\
\hline Notes & $\begin{array}{l}\text { Only core-period included in this meta-analysis as placebo group was } \\
\text { changed to sitagliptin. } \\
\text { ClinicalTrials.gov identifier: NCT01106690 } \\
\text { Extension study of } 26 \text { weeks, placebo changed to sitagliptin for } \\
\text { another } 26 \text { weeks. We only use data from the first } 26 \text {-week double- } \\
\text { blinded period. Patients and study center and local sponsor } \\
\text { personnel remained blinded throughout the extension period. }\end{array}$ \\
\hline
\end{tabular}

Risk of bias table

\begin{tabular}{|l|l|l||}
\hline Bias & $\begin{array}{l}\text { Authors' } \\
\text { judgement }\end{array}$ & Support for judgement \\
\hline $\begin{array}{l}\text { Random sequence } \\
\text { generation } \\
\text { (selection bias) }\end{array}$ & Low risk & $\begin{array}{l}\text { Interactive voice response system or interactive web } \\
\text { response system. Randomization was balanced using } \\
\text { permuted blocks of six patients per block and stratified } \\
\text { according to:(i) whether a patient entered the AHA }\end{array}$ \\
\hline
\end{tabular}




\begin{tabular}{|c|c|c|}
\hline & & $\begin{array}{l}\text { adjustment period and (ii) dose of pioglitazone at } \\
\text { randomization. }\end{array}$ \\
\hline $\begin{array}{l}\text { Allocation } \\
\text { concealment } \\
\text { (selection bias) }\end{array}$ & Unclear risk & $\begin{array}{l}\text { Not described but implied in details of randomization } \\
\text { which used an interactive voice response system or } \\
\text { interactive web response system. }\end{array}$ \\
\hline $\begin{array}{l}\text { Blinding of } \\
\text { participants and } \\
\text { personnel } \\
\text { (performance bias) }\end{array}$ & Low risk & $\begin{array}{l}\text { Double blind "patients received single-blind placebo } \\
\text { capsules matching study drug once daily" }\end{array}$ \\
\hline $\begin{array}{l}\text { Blinding of outcome } \\
\text { assessment } \\
\text { (detection bias) }\end{array}$ & Low risk & $\begin{array}{l}\text { Double blind "After completion of the core treatment } \\
\text { period, the database was locked and the study was } \\
\text { unblinded by the sponsor for regulatory filing. Patients } \\
\text { and study center and local sponsor personnel remained } \\
\text { blinded throughout the extension period." }\end{array}$ \\
\hline $\begin{array}{l}\text { Incomplete } \\
\text { outcome data } \\
\text { (attrition bias) }\end{array}$ & Low risk & $\begin{array}{l}\text { 296/344 completed the } 26 \text { week 'core' period. } \\
\text { Efficacy endpoints at week } 26 \text { were assessed using the } \\
\text { modified intention-to-treat (mITT) population. Drop outs } \\
\text { and losses to follow up accounted for. }\end{array}$ \\
\hline $\begin{array}{l}\text { Selective reporting } \\
\text { (reporting bias) }\end{array}$ & Unclear risk & No safety data for week 26 . \\
\hline Other bias & Unclear risk & $\begin{array}{l}\text { This study was sponsored by Janssen Research \& } \\
\text { Development, LLC. Editorial support was provided by } \\
\text { Janssen Global Services LLC. }\end{array}$ \\
\hline
\end{tabular}

\section{Gonzalez 2013}

\begin{tabular}{||l|l|}
\hline Methods & $\begin{array}{l}26 \text { week randomized, double-blind, four-arm, parallel group, Phase } 3 \\
\text { study was conducted at } 169 \text { centers in } 22 \text { countries. }\end{array}$ \\
\hline Participants & $\begin{array}{l}\text { Initially randomized } n=1284: \text { canagliflozin } 100 \mathrm{mg} n=368 ; \\
\text { canagliflozin } 300 \mathrm{mg} n=367 ; \text { sitagliflozin } n=366 ; \text { placebo } n=183 .\end{array}$ \\
\hline Interventions & $\begin{array}{l}\text { Canagliflozin } 100 \mathrm{mg} \text { or } 300 \mathrm{mg} \text {, sitagliptin } 100 \mathrm{mg} \text { or placebo } \\
(2: 2: 2: 1) \text { once daily for } 26 \text { weeks (oral dose) } \\
\text { Add on: metformin monotherapy. } \\
\text { Glycemic rescue therapy with glimepiride permitted. }\end{array}$ \\
\hline
\end{tabular}




\begin{tabular}{|c|c|}
\hline Outcomes & $\begin{array}{l}\text { Primary outcomes of the trial } \\
\text { reduction of HbA1c from baseline to week } 26 . \\
\text { Secondary outcomes of the trial } \\
\text { proportion of participants reaching HbA1c <7.0\% (53 mmol/mol), } \\
\text { change in FPG, } \\
2 \mathrm{~h} \text { postprandial glucose (PPG) } \\
\text { systolic BP } \\
\text { percent change in body weight, } \\
\text { triacylglycerol (i.e. triglycerides) and HDL-cholesterol, } \\
\text { all participants underwent a mixed-meal tolerance test (MMTT) } \\
\text { Adverse effects } \\
\text { AE reports, } \\
\text { safety laboratory tests, } \\
\text { vital sign measurements, } \\
\text { physical examinations, } \\
\text { SMBG and } 12 \text {-lead electrocardiograms. } \\
\text { urinary tract infections (UTIs) } \\
\text { genital mycotic infections. } \\
\text { documented episodes of hypoglycemia. }\end{array}$ \\
\hline Inclusion criteria & $\begin{array}{l}\text { men and women } \\
\text { with T2DM } \\
\text { aged } \geq 18 \text { and } \leq 80 \text { years, } \\
\text { inadequate glycemic control ( } \mathrm{HbA} 1 \mathrm{c} \geq 7.0 \% \text { ( } 53 \mathrm{mmol} / \mathrm{mol} \text { ) and } \\
\leq 10.5 \% \text { ( } 91 \mathrm{mmol} / \mathrm{mol}) \text { ) } \\
\text { on stable metformin therapy }(\geq 2,000 \mathrm{mg} / \mathrm{day} \text { (or } \geq 1,500 \mathrm{mg} / \text { day if } \\
\text { unable to tolerate higher dose) for } 8 \mathrm{weeks} \\
\text { fasting plasma glucose }(\mathrm{FPG})<15 \mathrm{mmol} / \mathrm{l} \text { at week } 2 \\
\text { fasting fingerstick glucose } \geq 6.1 \mathrm{mmol} / \mathrm{l} \text { and }<15 \mathrm{mmol} / \mathrm{l} \text { on day } 1 \text {. }\end{array}$ \\
\hline Exclusion criteria & $\begin{array}{l}\text { repeated FPG and/or fasting self-monitored blood glucose (SMBG) } \\
\geq 15.0 \mathrm{mmol} / \mathrm{I} \text { during the pretreatment phase, } \\
\text { history of type } 1 \text { diabetes, } \\
\text { cardiovascular disease (including myocardial infarction, unstable } \\
\text { angina, revascularization procedure or cerebrovascular accident) in } \\
\text { the } 3 \text { months before screening or uncontrolled hypertension, } \\
\text { treatment with a peroxisome proliferator-activated receptor } \gamma \\
\text { agonist, insulin, another SGLT2 inhibitor or any other AHA (except } \\
\text { metformin as monotherapy or in combination with a sulfonylurea) } \\
\text { in the } 12 \text { weeks before screening, } \\
\text { eGFR }<55 \mathrm{ml} \mathrm{min}^{-1}\left(1.73 \mathrm{~m}^{2}\right)^{-1} \text { (or }<60 \mathrm{ml} \mathrm{min-1}(1.73 \mathrm{m2})^{-1} \text { if }\end{array}$ \\
\hline
\end{tabular}




\begin{tabular}{||l||l||}
\hline \hline Notes & $\begin{array}{l}\text { based upon restriction in local label) or serum creatinine } \geq 124 \\
\mu \mathrm{mol} / \mathrm{l} \text { (men) or } \geq 115 \mu \mathrm{mol} / \mathrm{I} \text { (women). }\end{array}$ \\
\hline \hline & $\begin{array}{l}\text { ClinicalTrials.gov identifier: NCT01106677 } \\
\text { Study continued for } 26 \text { weeks after initial 26-week period, placebo } \\
\text { group switched to sitagliflozin (second period not included in our } \\
\text { analyses). }\end{array}$ \\
\hline
\end{tabular}

\section{Risk of bias table}

\begin{tabular}{|c|c|c|}
\hline Bias & \begin{tabular}{|l} 
Authors' \\
judgement
\end{tabular} & Support for judgement \\
\hline $\begin{array}{l}\text { Random sequence } \\
\text { generation (selection } \\
\text { bias) }\end{array}$ & Low risk & $\begin{array}{l}\text { The computer-generated randomization schedule was } \\
\text { prepared by the sponsor before the study. }\end{array}$ \\
\hline $\begin{array}{l}\text { Allocation } \\
\text { concealment } \\
\text { (selection bias) }\end{array}$ & Unclear risk & $\begin{array}{l}\text { The computer-generated randomization schedule was } \\
\text { prepared by the sponsor before the study. }\end{array}$ \\
\hline $\begin{array}{l}\text { Blinding of } \\
\text { participants and } \\
\text { personnel } \\
\text { (performance bias) }\end{array}$ & Low risk & $\begin{array}{l}\text { After randomization, } \mathrm{HbA} 1 \mathrm{c} \text { and FPG values were } \\
\text { masked to the study centers unless they met glycemic } \\
\text { rescue criteria. }\end{array}$ \\
\hline $\begin{array}{l}\text { Blinding of outcome } \\
\text { assessment } \\
\text { (detection bias) }\end{array}$ & Low risk & $\begin{array}{l}\text { After completion of period I (first } 26 \text { weeks), the } \\
\text { database was locked and the study was unblinded by } \\
\text { the sponsor for regulatory filing; }\end{array}$ \\
\hline $\begin{array}{l}\text { Incomplete outcome } \\
\text { data (attrition bias) }\end{array}$ & Low risk & $\begin{array}{l}\text { 1119/1284 completed the first } 26 \text {-week period. } \\
\text { All drop outs and losses to follow up accounted for. } \\
\text { Primary efficacy analysis was performed in the modified } \\
\text { intent-to-treat (mITT) population (randomized } \\
\text { participants who received } 1 \text { dose of study drug) using a } \\
\text { last observation carried forward (LOCF) approach. } \\
\text { Primary efficacy analyses were performed in the mITT } \\
\text { population according to randomized treatment } \\
\text { assignment using LOCF to impute missing data; for } \\
\text { participants who received rescue therapy, the last post- } \\
\text { baseline value before rescue was used. }\end{array}$ \\
\hline
\end{tabular}




\begin{tabular}{|l|l||l||}
\hline $\begin{array}{l}\text { Selective reporting } \\
\text { (reporting bias) }\end{array}$ & Low risk & All major clinically relevant outcomes were reported \\
\hline Other bias & Unclear risk & $\begin{array}{l}\text { This study was supported by Janssen Research \& } \\
\text { Development, LLC who contributed to clinical } \\
\text { management, data review and preparation of the study } \\
\text { report. }\end{array}$ \\
\hline
\end{tabular}

\section{Haring 2014}

\begin{tabular}{|c|c|}
\hline Methods & $\begin{array}{l}\text { 76-week, randomized, double-blind, placebo-controlled, parallel- } \\
\text { group study in } 148 \text { centers across } 12 \text { countries }\end{array}$ \\
\hline Participants & $\begin{array}{l}638 \text { initially randomized: (empagliflozin } 10 \mathrm{mg}, \mathrm{n}=217 \\
\text { empagliflozin } 25 \mathrm{mg}, \mathrm{n}=214 \text {; placebo, } \mathrm{n}=207 \text { ) }\end{array}$ \\
\hline Interventions & $\begin{array}{l}\text { Randomized (1:1:1) to receive once-daily empagliflozin } 10 \mathrm{mg} \text {, } \\
\text { empagliflozin } 25 \mathrm{mg} \text {, or placebo. } \\
\text { Add on: metformin } \\
\text { Rescue mediation permitted in case of hyperglycemia. }\end{array}$ \\
\hline Outcomes & $\begin{array}{l}\text { Primary outcomes of the trial } \\
\text { change from baseline in HbA1c at week } 24 \text {. } \\
\text { Secondary outcomes of the trial } \\
\text { change from baseline to week } 24 \text { in body weight, } \\
\text { weighted mean daily glucose (MDG) level using an } 8 \text {-point blood } \\
\text { glucose profile, } \\
\text { percentage of patients with baseline } \mathrm{HbA} 1 \mathrm{c} \text { level } \geq 7.0 \% \text { ( } \geq 53 \\
\text { mmol/mol) who had an HbA1c level }<7 \% \text { ( }<53 \text { mmol/mol) at week } \\
24, \\
\text { changes from baseline to week } 24 \text { in fasting plasma glucose (FPG), } \\
\text { waist circumference, } \\
\text { systolic BP (SBP), } \\
\text { diastolic BP (DBP), } \\
\text { percentage of patients with }>5 \% \text { reduction in body weight at week } \\
24 ; \\
\text { percentage of patients with uncontrolled BP at baseline who had } \\
\text { controlled BP (SBP }<130 \text { and DBP }<80 \text { mmHg) at week } 24 \\
\text { use of rescue medication, } \\
\text { change from baseline in } 2 \text {-h postprandial glucose (PPG) was } \\
\text { assessed in a subset of patients based on a meal tolerance test }\end{array}$ \\
\hline
\end{tabular}




\begin{tabular}{|c|c|}
\hline & $\begin{array}{l}\text { (MTT) at baseline and week } 24 \text {. } \\
\text { Adverse events and safety assessments } \\
\text { vital signs, } \\
\text { clinical laboratory parameters, } \\
\text { 12-lead electrocardiogram findings, } \\
\text { adverse events (all), } \\
\text { all events with an onset after the first dose of trial medication up } \\
\text { to a period of } 7 \text { days after the last dose, } \\
\text { confirmed hypoglycemic AEs (plasma glucose } \leq 3.9 \text { mmol/L and/or } \\
\text { requiring assistance), } \\
\text { suspected urinary tract infection (UTI), } \\
\text { genital infection. }\end{array}$ \\
\hline Inclusion criteria & $\begin{array}{l}\text { adults aged } \geq 18 \text { years, } \\
\mathrm{T} 2 \mathrm{DM} \text {, } \\
\mathrm{BMI} \leq 45 \mathrm{~kg} / \mathrm{m} 2 \text { ) with inadequately controlled type } 2 \text { diabetes } \\
\text { ( } \mathrm{HbA} 1 \mathrm{c} \geq 7 \% \text { to } \leq 10 \% \text { ( } \geq 53 \text { to } \leq 86 \mathrm{mmol} / \mathrm{mol} \text { )) despite undergoing a } \\
\text { diet and exercise program and a stable immediate release } \\
\text { metformin regimen (unchanged for } \geq 12 \text { weeks prior to } \\
\text { randomization). }\end{array}$ \\
\hline Exclusion criteria & $\begin{array}{l}\text { uncontrolled hyperglycemia (glucose level }>13.3 \mathrm{mmol} / \mathrm{L} \text { ) after an } \\
\text { overnight fast confirmed by a second measurement, acute } \\
\text { coronary syndrome, } \\
\text { stroke, or transient ischemic attack within } 3 \text { months prior to } \\
\text { informed consent, } \\
\text { indication of liver disease (alanine aminotransferase, alkaline } \\
\text { aminotransferase, or alkaline phosphatase levels more than three } \\
\text { times the upper limit of normal) } \\
\text { impaired kidney function (estimated eGFR }<30 \mathrm{~mL} / \mathrm{min} / 1.73 \mathrm{~m} 2 \text { ) } \\
\text { during screening or run-in } \\
\text { contraindications to metformin according to the local label } \\
\text { bariatric surgery or other gastrointestinal surgeries that induce } \\
\text { chronic malabsorption } \\
\text { medical history of cancer (except for basal cell carcinoma) or } \\
\text { treatment for cancer within the last } 5 \text { years } \\
\text { blood dyscrasias or any disorders causing hemolysis or unstable } \\
\text { erythrocytes, } \\
\text { treatment with anti-obesity drugs } 3 \text { months prior to consent, } \\
\text { use of any treatment at screening leading to unstable body weight } \\
\text { treatment with systemic steroids at the time of consent } \\
\text { change in the dosage of thyroid hormones within } 6 \text { weeks prior to } \\
\text { consent, } \\
\text { alcohol or drug abuse within } 3 \text { months of consent }\end{array}$ \\
\hline
\end{tabular}




\begin{tabular}{||l||l||}
\hline \hline Notes & $\begin{array}{l}\text { investigational drug intake in another trial within } 30 \text { days prior to } \\
\text { the current trial. }\end{array}$ \\
\hline \hline $\begin{array}{l}\text { ClinicalTrials.gov identifier: NCT01159600 } \\
\text { Haring } 2014 \text { includes data from Merker 2015, a double-blind } \\
\text { extension trial for } \geq 52 \text { weeks i.e. } 76 \text { weeks in total. }\end{array}$ \\
\hline
\end{tabular}

\section{Risk of bias table}

\begin{tabular}{|c|c|c|}
\hline Bias & $\begin{array}{l}\text { Authors' } \\
\text { judgement }\end{array}$ & Support for judgement \\
\hline $\begin{array}{l}\text { Random sequence } \\
\text { generation (selection } \\
\text { bias) }\end{array}$ & Low risk & $\begin{array}{l}\text { Randomization was performed using a third-party } \\
\text { interactive voice and web response system, and was } \\
\text { stratified by HbA1c level and eGFR. }\end{array}$ \\
\hline $\begin{array}{l}\text { Allocation } \\
\text { concealment } \\
\text { (selection bias) }\end{array}$ & Unclear risk & $\begin{array}{l}\text { Not described, but implied in the computer-generated } \\
\text { stratified randomization sequence technique. }\end{array}$ \\
\hline $\begin{array}{l}\text { Blinding of } \\
\text { participants and } \\
\text { personnel } \\
\text { (performance bias) }\end{array}$ & Low risk & Double-blind. \\
\hline $\begin{array}{l}\text { Blinding of outcome } \\
\text { assessment (detection } \\
\text { bias) }\end{array}$ & Low risk & Double-blind. \\
\hline $\begin{array}{l}\text { Incomplete outcome } \\
\text { data (attrition bias) }\end{array}$ & Low risk & $\begin{array}{l}\text { 591/638 completed. One patient assigned to receive } \\
\text { empagliflozin } 25 \text { mg was not treated The FAS } \\
\text { comprised } 637 \text { patients, who were treated with one or } \\
\text { more doses of study drug and who had a baseline } \\
\text { HbA1c value. }\end{array}$ \\
\hline $\begin{array}{l}\text { Selective reporting } \\
\text { (reporting bias) }\end{array}$ & Low risk & $\begin{array}{l}\text { All clinically relevant outcomes are defined and } \\
\text { reported. }\end{array}$ \\
\hline Other bias & Unclear risk & $\begin{array}{l}\text { This research was supported by Boehringer Ingelheim } \\
\text { and Eli Lilly. Medical writing assistance was supported } \\
\text { by Boehringer Ingelheim. Some of the trialists are } \\
\text { employees of Boehringer Ingelheim. }\end{array}$ \\
\hline
\end{tabular}


Henry 2012

\begin{tabular}{|c|c|}
\hline Methods & $\begin{array}{l}\text { 24-week, randomized, double-blind three-arm active-controlled } \\
\text { studies conducted at } 131 \text { sites in } 4 \text { locations, North America, Latin } \\
\text { America, Europe and Asia. }\end{array}$ \\
\hline Participants & $\begin{array}{l}641 \text { initially randomized: dapagliflozin } 10 \text { mg plus metformin } n=211 \text {; } \\
\text { dapagliflozin } 10 \text { mg plus placebo } n=219 ; \text { dapagliflozin } 10 \mathrm{mg} \\
\text { metformin plus placebo } n=208\end{array}$ \\
\hline Interventions & $\begin{array}{l}\text { Randomly assigned (1:1:1). Dapagliflozin } 10 \mathrm{mg} \text { plus metformin XR } \\
\text { (combination), dapagliflozin } 10 \mathrm{mg} \text { plus placebo (dapagliflozin), and } \\
\text { metformin XR plus placebo (metformin). } \\
\text { Drug administration occurred with the evening meal. } \\
\text { Add on: diet and exercise counselling. } \\
\text { Rescue medication permitted. }\end{array}$ \\
\hline Outcomes & $\begin{array}{l}\text { Primary outcomes of the trial } \\
\text { HbA1c change from baseline at week } 24 \text {. } \\
\text { Secondary outcomes of the trial } \\
\text { change from baseline at week } 24 \text { in fasting plasma glucose, } \\
\text { proportion of patients achieving a therapeutic glycemic response } \\
\text { (HbA1c }<7 \% \text { ), } \\
\text { HbA1c for patients with baseline HbA1c } \geq 9 \% \text {, } \\
\text { total body weight, } \\
\text { proportion discontinued or rescued for failing to achieve } \\
\text { prespecified glycemic targets based on prespecified rescue criteria, } \\
\text { non-inferiority of dapagliflozin } \\
10 \text { mg to metformin XR for changes in fasting } \\
\text { plasma glucose ( } 0.83 \text { mmol / I margin) and HbA1c ( } 0.35 \% \text { margin); } \\
\text { if non-inferiority was demonstrated } \\
\text { superiority of dapagliflozin } 10 \text { mg was tested, } \\
\text { difference in weight reduction with dapagliflozin } 10 \text { mg vs. } \\
\text { metformin } \\
\text { Adverse events and safety assessments } \\
\text { vital signs, } \\
\text { laboratory measurements } \\
\text { adverse events } \\
\text { vulvovaginitis, balanitis and related genital infection. }\end{array}$ \\
\hline
\end{tabular}




\begin{tabular}{|c|c|}
\hline Inclusion criteria & $\begin{array}{l}\text { men and women } \\
\text { T2DM uncontrolled by diet and exercise } \\
18-77 \text { years, } \\
\text { hemoglobin } \mathrm{A} 1 \mathrm{c}(\mathrm{HbA} 1 \mathrm{c}) 7.5-12 \% \text {, } \\
\text { body mass index } \leq 45 \mathrm{~kg} / \mathrm{m}^{2}, \\
\text { C-peptide concentration } \geq 0.33 \mathrm{nmol} / \mathrm{I}\end{array}$ \\
\hline Exclusion criteria & $\begin{array}{l}\text { serum creatinine } \geq 132.60 \mu \mathrm{mol} / \mathrm{I} \text { (men) or } \geq 123.76 \mu \mathrm{mol} / \mathrm{I} \\
\text { (women) consistent with metformin labelling, } \\
\text { urine albumin:creatinine ratio }>1800 \mathrm{mg} / \mathrm{g} \text {, } \\
\text { serum aspartate transaminase or alanine, } \\
\text { transaminase }>3 \text { times upper limit of normal (ULN), } \\
\text { creatine kinase }>3 \text { times ULN, } \\
\text { history of diabetes insipidus, } \\
\text { symptoms of poorly controlled diabetes (including marked polyuria } \\
\text { and polydipsia with }>10 \% \text { weight loss during } 3 \text { months before } \\
\text { enrolment), } \\
\text { clinically significant renal, hepatic, hematological, oncological, } \\
\text { endocrine, psychiatric or rheumatic disease, } \\
\text { a cardiovascular event within } 6 \text { months or New York Heart } \\
\text { Association Class III or IV congestive heart failure } \\
\text { systolic blood pressure } \geq 180 \text { or diastolic blood pressure } \geq 110 \\
\text { mmHg. }\end{array}$ \\
\hline Notes & $\begin{array}{l}\text { Three arms, metformin + placebo vs. dapagliflozin + placebo } \\
\text { included. Dapa+ metformin not included in our analyses. } \\
\text { ClinicalTrials.gov identifier: Study } 2 \text { (NCT00859898), only data from } \\
\text { study } 2 \text { (10mg dapagliflozin) are included in our analyses. }\end{array}$ \\
\hline
\end{tabular}

\section{Risk of bias table}

\begin{tabular}{||l|l|l||}
\hline Bias & $\begin{array}{l}\text { Authors' } \\
\text { judgement }\end{array}$ & Support for judgement \\
\hline $\begin{array}{l}\text { Random sequence } \\
\text { generation } \\
\text { (selection bias) }\end{array}$ & Low risk & $\begin{array}{l}\text { Random numbers (stratified by site, in blocks of three, } \\
\text { and randomly assigned (1:1:1) to double-blinded groups } \\
\text { of combination therapy, dapagliflozin monotherapy and } \\
\text { metformin monotherapy by IVRS.) }\end{array}$ \\
\hline $\begin{array}{l}\text { Allocation } \\
\text { concealment } \\
\text { (selection bias) }\end{array}$ & Unclear risk & $\begin{array}{l}\text { Not stated, but implied by randomization using a central } \\
\text { interactive voice response system. }\end{array}$ \\
\hline $\begin{array}{l}\text { Blinding of } \\
\text { participants and }\end{array}$ & Low risk & Double-blind. \\
\hline
\end{tabular}




\begin{tabular}{|c|c|c|}
\hline $\begin{array}{l}\text { personnel } \\
\text { (performance bias) }\end{array}$ & & \\
\hline $\begin{array}{l}\text { Blinding of outcome } \\
\text { assessment } \\
\text { (detection bias) }\end{array}$ & Low risk & Double-blind. \\
\hline $\begin{array}{l}\text { Incomplete } \\
\text { outcome data } \\
\text { (attrition bias) }\end{array}$ & Low risk & $\begin{array}{l}\text { 552/641completed. Primary efficacy data were derived } \\
\text { from randomized patients with } \geq 1 \text { dose of double-blind } \\
\text { medication, and with baseline and } \geq 1 \text { post baseline } \\
\text { measurements. Last observation carried forward. All } \\
\text { drop outs and losses to follow up accounted for. }\end{array}$ \\
\hline $\begin{array}{l}\text { Selective reporting } \\
\text { (reporting bias) }\end{array}$ & Low risk & All clinically relevant outcomes are defined and reported. \\
\hline Other bias & Unclear risk & $\begin{array}{l}\text { These studies were funded by Bristol-Myers Squibb and } \\
\text { AstraZeneca. Employees of Bristol-Myers Squibb } \\
\text { provided writing and editorial assistance. }\end{array}$ \\
\hline
\end{tabular}

Häring 2013

\begin{tabular}{|c|c|}
\hline Methods & $\begin{array}{l}\text { 76-week, randomized, double-blind, placebo-controlled trial in } 148 \\
\text { centers in } 12 \text { countries }\end{array}$ \\
\hline Participants & 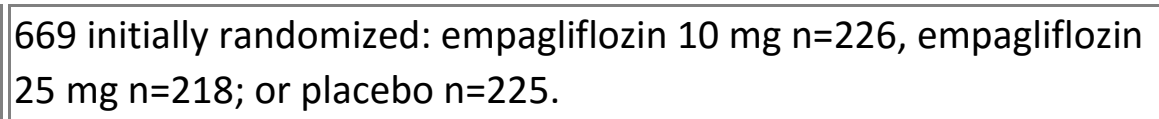 \\
\hline Interventions & $\begin{array}{l}\text { Randomized (1:1:1) to receive once-daily oral dose empagliflozin } 10 \\
\mathrm{mg} \text {, empagliflozin } 25 \mathrm{mg} \text {, versus placebo therapy to metformin } \\
\text { ( } \geq 1,500 \mathrm{mg} / \text { day or maximum tolerated dose or maximum dose } \\
\text { according to local label) } \\
\text { Add on: metformin, sulfonylurea (greater than or equal to half the } \\
\text { maximum recommended dose, or the maximum tolerated dose, or } \\
\text { the maximum dose according to local label). }\end{array}$ \\
\hline Outcomes & $\begin{array}{l}\text { Primary outcomes of the trial } \\
\text { change from baseline in } \mathrm{HbA} 1 \mathrm{c} \text { at week } 24 \text {. } \\
\text { Secondary outcomes of the trial } \\
\text { change from baseline to week } 24 \text { in body weight and } \\
\text { mean daily glucose (MDG) using an } 8 \text {-point blood glucose profile. } \\
\text { percentage with baseline } \mathrm{HbA} 1 \mathrm{c} \geq 7.0 \% \text { who had } \mathrm{HbA} 1 \mathrm{c}<7 \% \text { at }\end{array}$ \\
\hline
\end{tabular}




\begin{tabular}{|c|c|}
\hline & $\begin{array}{l}\text { week 24; change from baseline in fasting plasma glucose (FPG), } \\
\text { waist circumference, } \\
\text { systolic and diastolic blood pressure (SBP and DBP) } \\
\text { percentage of patients with .5\% reduction in body weight at week } \\
24 \\
\text { use of rescue medication. } \\
\text { change from baseline in } 2 \mathrm{~h} \text { postprandial glucose (PPG) was } \\
\text { assessed in a subset of patients based on a meal tolerance test } \\
\text { (MTT) performed } \\
\text { Adverse events and safety assessments } \\
\text { vital signs, } \\
\text { clinical laboratory parameters, } \\
\text { 12-lead electrocardiogram, } \\
\text { confirmed hypoglycemic AEs (plasma glucose } \leq 3.9 \text { mmol/L and/or } \\
\text { requiring assistance) } \\
\text { urinary tract infection (UTI) and genital infection. }\end{array}$ \\
\hline Inclusion criteria & $\begin{array}{l}\text { aged } \geq 18 \text { years } \\
\text { BMI } \leq 45 \mathrm{~kg} / \mathrm{m}^{2} \\
\text { inadequately controlled type } 2 \text { diabetes ( } \mathrm{HbA} 1 \mathrm{c} \geq 7 \text { to } \leq 10 \% \text { ) } \\
\text { despite a diet and exercise program } \\
\text { on stable metformin immediate release plus a sulfonylurea for at } \\
\text { least } 12 \text { weeks prior }\end{array}$ \\
\hline Exclusion criteria & $\begin{array}{l}\text { Exclusion criteria } \\
\text { uncontrolled hyperglycemia (glucose level }>13.3 \mathrm{mmol} / \mathrm{L} \text { ) after an } \\
\text { overnight fast, confirmed by a second measurement), } \\
\text { acute coronary syndrome, stroke or transient ischemic attack } \\
\text { within } 3 \text { months prior to consent, } \\
\text { indication of liver disease, } \\
\text { impaired kidney function (estimated glomerular filtration rate } \\
\text { (eGFR) }<30 \mathrm{~mL} / \mathrm{min} / 1.73 \mathrm{~m}^{2} \text { ) } \\
\text { contraindications to metformin or sulfonylurea, } \\
\text { gastrointestinal surgeries that induce chronic malabsorption, } \\
\text { history of cancer (except basal cell carcinoma) or treatment for } \\
\text { cancer within } 5 \text { years, } \\
\text { blood dyscrasias or any disorders causing hemolysis or unstable } \\
\text { erythrocytes, } \\
\text { treatment with anti-obesity drugs } 3 \text { months prior to consent, } \\
\text { use of any treatment at screening that leads to unstable body } \\
\text { weight, } \\
\text { treatment with systemic steroids at time of consent, } \\
\text { change in dosage of thyroid hormones within } 6 \text { weeks of consent, }\end{array}$ \\
\hline
\end{tabular}




\begin{tabular}{||l||l||}
\hline \hline Notes & $\begin{array}{l}\text { alcohol or drug abuse } \\
\text { investigational drug intake within } 30 \text { days of the trial. }\end{array}$ \\
\hline $\begin{array}{l}\text { The open label arm in Häring } 2013 \text { including patients with HbAb1c } \\
10 \% \text { is not included in the analyses in this review. } \\
\text { ClinicalTrials.gov identifier: NCT01159600. The 52-week extension } \\
\text { data (i.e. } 76 \text { weeks in total) from } \underline{\text { Häring } 2015} \text { are included in the } \\
\text { analyses. }\end{array}$ \\
\hline
\end{tabular}

\section{Risk of bias table}

\begin{tabular}{|l|l|l||}
\hline Bias & $\begin{array}{l}\text { Authors' } \\
\text { judgement }\end{array}$ & Support for judgement \\
\hline $\begin{array}{l}\text { Random sequence } \\
\text { generation (selection } \\
\text { bias) }\end{array}$ & Low risk & Third-party interactive voice and web response \\
system and was stratified by HbA1c.
\end{tabular}




\begin{tabular}{|c|c|}
\hline Methods & $\begin{array}{l}12 \text { week, multicenter, randomized, placebo-controlled, parallel- } \\
\text { group study }\end{array}$ \\
\hline Participants & $\begin{array}{l}383 \text { initially randomized. } 300 m g \text { canagliflozin } n=75 ; 200 m g \\
\text { canagliflozin } n=77 ; 100 m g \text { canagliflozin } n=74 ; \text { placebo } n=75\end{array}$ \\
\hline Interventions & $\begin{array}{l}\text { Canagliflozin (50,100, } 200 \text { or } 300 \mathrm{mg} \text { once daily (oral dose) versus } \\
\text { placebo for } 12 \text { weeks. } \\
\text { Eligible patients were randomized using a block allocation method } \\
\text { into one of five groups (ratio 1:1:1:1:1) to receive placebo or one of } \\
\text { the four doses of canagliflozin. } \\
\text { Add on: diet and exercise therapy. }\end{array}$ \\
\hline Outcomes & $\begin{array}{l}\text { Primary outcomes of the trial } \\
\text { change in HbA1c from the last day of the run-in period (baseline) to } \\
\text { the end of the treatment period ( } 12 \text { weeks). } \\
\text { Secondary outcomes of the trial } \\
\text { change in FPG, } \\
\text { percentages of patients with HbA1c }<7.0 \% \text {, } \\
\text { changes in urinary glucose/creatinine ratio, } \\
\text { body weight, } \\
\text { BMI, } \\
\text { waist circumference, } \\
\text { lipid levels, } \\
\text { blood pressure, } \\
\text { insulin and proinsulin levels, } \\
\text { homeostasis model assessment of } \beta \text {-cell function (HOMA- } \beta \text { ) } \\
\text { meal tolerance-related parameters. } \\
\text { Adverse events and safety assessments } \\
\text { AEs and safety assessments (AEs were classified according to } \\
\text { system organ class in terms of their potential relationship with the } \\
\text { study drug (no causal relationship or possible causal relationship) } \\
\text { and severity (mild, moderate or severe), } \\
\text { vital signs, } \\
12 \text {-lead electrocardiography, } \\
\text { clinical laboratory tests (blood chemistry, hematology, coagulation, } \\
\text { bone markers and urinalysis), } \\
\text { hypoglycemic symptoms. }\end{array}$ \\
\hline Inclusion criteria & $\begin{array}{l}\text { men and women } \\
\text { T2DM for at least } 3 \text { months before the run-in period, }\end{array}$ \\
\hline
\end{tabular}




\begin{tabular}{||l||l||}
\hline \hline & $\begin{array}{l}\text { HbA1c levels of } 6.9-9.9 \% \text { at the start of the run-in period, } \\
\text { to have undergone diet and exercise therapy, with no change in } \\
\text { their regimen for } 18 \text { weeks before the study. } \\
\text { aged } 20-80 \text { years } \\
\text { Japanese }\end{array}$ \\
\hline Exclusion criteria & $\begin{array}{l}\text { history of or current serious diabetic complications (e.g. } \\
\text { proliferative diabetic retinopathy, stage } 3 \text { or later overt } \\
\text { nephropathy, diabetic ketoacidosis or serious diabetic } \\
\text { neuropathy), } \\
\text { FPG } \geq 270 \text { mg/dl (1 mg/dl FPG=0.0555 mmol/l), } \\
\text { indication for insulin therapy, } \\
\text { hereditary glucose-galactose malabsorption } \\
\text { renal glycosuria. }\end{array}$ \\
\hline Notes & $\begin{array}{l}\text { ClinicalTrials.gov identifier: NCT01022112 } \\
\text { "In terms of concomitant treatments, antihyperglycemic drugs were } \\
\text { prohibited after randomization until the end of the follow-up period. } \\
\text { Diet and exercise interventions were to be continued without } \\
\text { modification after randomization until the end of the follow-up } \\
\text { period." } \\
\text { "Patient characteristics were generally well balanced among the five } \\
\text { groups, with no marked differences among treatment groups (Table } \\
\text { 1) except for height, body weight and presence/absence of } \\
\text { neuropathy or hypertension, which were unbalanced among the five } \\
\text { groups at p<0.15." }\end{array}$ \\
\hline \hline
\end{tabular}

\section{Risk of bias table}

\begin{tabular}{|l|l|l|l|}
\hline Bias & $\begin{array}{l}\text { Authors' } \\
\text { judgement }\end{array}$ & Support for judgement \\
\hline $\begin{array}{l}\text { Random sequence } \\
\text { generation } \\
\text { (selection bias) }\end{array}$ & Low risk & $\begin{array}{l}\text { Randomization was conducted by a central committee, } \\
\text { which provided the investigators at each site with } \\
\text { randomization codes stored in sealed envelopes. }\end{array}$ \\
\hline $\begin{array}{l}\text { Allocation } \\
\text { concealment } \\
\text { (selection bias) }\end{array}$ & Low risk & $\begin{array}{l}\text { The randomization code was stored in sealed envelopes } \\
\text { was not to be broken until data entry had been completed } \\
\text { or unless needed in an emergency. }\end{array}$ \\
\hline $\begin{array}{l}\text { Blinding of } \\
\text { participants and } \\
\text { personnel }\end{array}$ & Low risk & $\begin{array}{l}\text { Investigators and patients were blinded to the study drug } \\
\text { received during the treatment phase. }\end{array}$ \\
\hline
\end{tabular}




\begin{tabular}{|c|c|c|}
\hline (performance bias) & & \\
\hline $\begin{array}{l}\text { Blinding of } \\
\text { outcome } \\
\text { assessment } \\
\text { (detection bias) }\end{array}$ & Low risk & $\begin{array}{l}\text { Investigators and patients were blinded to the study drug } \\
\text { received during the treatment phase. }\end{array}$ \\
\hline $\begin{array}{l}\text { Incomplete } \\
\text { outcome data } \\
\text { (attrition bias) }\end{array}$ & Low risk & $\begin{array}{l}361 / 383 \text { completed (i.e. } 22 \text { withdrew after } \\
\text { randomization). All drops outs and losses to follow up } \\
\text { accounted for. } \\
\text { Primary and secondary analyses were conducted in the } \\
\text { full analysis set (FAS), defined as all allocated patients, } \\
\text { excluding patients who did not receive any study drug or } \\
\text { who did not have any efficacy data after entering the } \\
\text { treatment phase. In the event of missing data for efficacy } \\
\text { variables, the last observation carried forward (LOCF) } \\
\text { approach was used to impute missing values in the FAS } \\
\text { analyses. }\end{array}$ \\
\hline $\begin{array}{l}\text { Selective reporting } \\
\text { (reporting bias) }\end{array}$ & Low risk & All major and clinically relevant outcomes were reported \\
\hline Other bias & Unclear risk & $\begin{array}{l}\text { This study was funded by Mitsubishi Tanabe Pharma } \\
\text { Corporation, the manufacturer of canagliflozin. }\end{array}$ \\
\hline
\end{tabular}

Jabbour 2014

\begin{tabular}{|c|c|}
\hline Methods & $\begin{array}{l}\text { 24-week, randomized, double-blind, placebo-controlled, parallel- } \\
\text { group, phase } 3 \text { study, multicenter in } 6 \text { countries (Argentina, } \\
\text { Germany, Mexico, Poland, the United Kingdom, and the United } \\
\text { States) }\end{array}$ \\
\hline Participants & 451 initially randomized: dapagliflozin $n=225 ;$ placebo $n=226$. \\
\hline Interventions & $\begin{array}{l}\text { Dapagliflozin } 10 \mathrm{mg} \text { or placebo (oral dose, once daily) } \\
\text { Add on: metformin, sitagliptin. Participants in stratum } 1 \text {, study } \\
\text { treatment was added to sitagliptin monotherapy; in stratum } 2 \\
\text { received open-label oral metformin immediate release } 500 \mathrm{mg} \\
\text { tablets ( } \geq 1500 \mathrm{mg} / \text { day). } \\
\text { A rescue therapy, open-label oral glimepiride permitted } \leq 6 \mathrm{mg} / \mathrm{day} \text {, } \\
\text { was given to patients with FPG }>270 \mathrm{mg} / \mathrm{dl}(15.0 \mathrm{mmol} / \mathrm{l} \text { ) - weeks } 0- \\
4 ; \mathrm{FPG}>240 \mathrm{mg} / \mathrm{dl}(13.3 \mathrm{mmol} / \mathrm{l})-\text { weeks } 4-12 ; \text { or FPG }>200 \mathrm{mg} / \mathrm{dl}\end{array}$ \\
\hline
\end{tabular}




\begin{tabular}{|c|c|}
\hline & $\begin{array}{l}(11.1 \mathrm{mmol} / \mathrm{l}) \text { or } \mathrm{HbA} 1 \mathrm{c}>8.0 \% \text { (64 } \mathrm{mmol} / \mathrm{mol})- \text { weeks } 12-24 \text {. } \\
\text { Sitagliptin monotherapy was allowed only in countries where it was } \\
\text { approved. No other oral antidiabetic agents permitted. }\end{array}$ \\
\hline Outcomes & $\begin{array}{l}\text { Primary outcomes of the trial } \\
\text { change in HbA1c from baseline at week } 24 \text {. } \\
\text { Secondary outcomes of the trial } \\
\text { change in total body weight from baseline to week 24, } \\
\text { change in HbA1c in patients with baseline HbA1c } \geq 8 \% \text { ( } 64 \\
\text { mmol/mol), } \\
\text { change in FPG from baseline to week } 24, \text { change in seated SBP in } \\
\text { patients with baseline seated SBP } \geq 130 \text { mmHg from baseline to } \\
\text { week } 8, \\
\text { glycemic response rate (HbA1c reduction } \geq 0.7 \% \text { ( } 7.7 \text { mmol/mol) } \\
\text { from baseline), } \\
\text { change in } 2 \text {-h post liquid meal glucose (PPG) from baseline (day } 0 \text { ) } \\
\text { (PPG, C-peptide, and insulin determination), } \\
\text { proportion of subjects achieving a therapeutic glycemic response } \\
\text { (HbA1c <7.0\% (53 mmol/mol)), } \\
\text { change in seated SBP from baseline, } \\
\text { percent change in fasting lipids (total cholesterol, low-density } \\
\text { lipoprotein cholesterol, high-density lipoprotein cholesterol, and } \\
\text { triglycerides), } \\
\text { change in } \beta \text {-cell function (HOMA-2)), } \\
\text { insulin resistance (as measured by the HOMA for Insulin } \\
\text { Resistance). } \\
\text { Adverse events and safety assessments } \\
\text { reported adverse events (AEs), } \\
\text { laboratory values, } \\
\text { electrocardiogram, pulse, BP, } \\
\text { hypoglycemic events, } \\
\text { calculated creatinine clearance, } \\
\text { estimated glomerular filtration rate, } \\
\text { physical examination findings. }\end{array}$ \\
\hline Inclusion criteria & $\begin{array}{l}\text { men and women } \\
\geq 18 \text { years (An upper age limit was imposed for those receiving } \\
\text { metformin where local label restrictions applied). Mean age } \\
\text { approximately } 55 \text { years. } \\
\text { T2DM } \\
\text { HbA1c values between } 7.7 \%(61 \mathrm{mmol} / \mathrm{mol}) \text { and } 10.5 \% \text { (91 }\end{array}$ \\
\hline
\end{tabular}




\begin{tabular}{|c|c|}
\hline & $\begin{array}{l}\mathrm{mmol} / \mathrm{mol} \text { ) for individuals not receiving a DPP-4 inhibitor at } \\
\text { enrolment and between } 7.2 \% \text { ( } 55 \mathrm{mmol} / \mathrm{mol}) \text { and } 10.0 \% \text { ( } 86 \\
\mathrm{mmol} / \mathrm{mol} \text { ) for those receiving a DPP-4 inhibitor. } \\
\text { prior to randomization, } \mathrm{HbA} 1 \mathrm{c} \text { values were required to be between } \\
7.0 \% \text { ( } 53 \mathrm{mmol} / \mathrm{mol} \text { ) and } 10.0 \% \text { ( } 86 \mathrm{mmol} / \mathrm{mol} \text { ) for all patients. }\end{array}$ \\
\hline Exclusion criteria & $\begin{array}{l}\text { type } 1 \text { diabetes } \\
\text { fasting plasma glucose (FPG) }>270 \mathrm{mg} / \mathrm{dL} \text { ( } 15.0 \mathrm{mmol} / \mathrm{I}) \\
\text { pregnant or breast-feeding women } \\
\text { treatment with OADs other than metformin or DPP-4 inhibitors } \\
\text { within the } 10 \text { weeks prior to enrolment } \\
\text { receiving metformin with a calculated creatinine clearance }<60 \\
\mathrm{ml} / \mathrm{min} \text { or serum creatinine values } \geq 1.5 \mathrm{mg} / \mathrm{dl} \text { for men or } \geq 1.4 \\
\mathrm{mg} / \mathrm{dl} \text { for women } \\
\text { if not treated with metformin and with a baseline calculated } \\
\text { creatinine clearance }<50 \mathrm{ml} / \mathrm{min} \mathrm{SBP} \geq 170 \mathrm{mmHg} \text { and/or diastolic } \\
\text { BP (DBP) } \geq 110 \mathrm{mmHg} \text { (at randomization SBP }<160 \mathrm{mmHg} \text { and/or a } \\
\text { DBP }<100 \mathrm{mmHg} \text { ) }\end{array}$ \\
\hline \multirow[t]{2}{*}{ Notes } & ClinicalTrials.gov identifier: NCT00984867 \\
\hline & $\begin{array}{l}\text { 24-week blinded extension period (not included in our analyses as it } \\
\text { was only site- and patient-blind). }\end{array}$ \\
\hline
\end{tabular}

Risk of bias table

\begin{tabular}{|c|c|c|}
\hline Bias & \begin{tabular}{|l|} 
Authors' \\
judgement
\end{tabular} & Support for judgement \\
\hline $\begin{array}{l}\text { Random sequence } \\
\text { generation } \\
\text { (selection bias) }\end{array}$ & Low risk & $\begin{array}{l}\text { Not described, but use of stratification implies } \\
\text { computerized method. } \\
\text { Randomized patients were stratified by concomitant } \\
\text { metformin use at baseline: in stratum } 1 \text {, study treatment } \\
\text { was added to sitagliptin monotherapy; in stratum } 2 \text {, study } \\
\text { treatment was added to sitagliptin plus metformin IR } \\
(\geq 1500 \mathrm{mg} / \text { day, administered BID with meals). }\end{array}$ \\
\hline $\begin{array}{l}\text { Allocation } \\
\text { concealment } \\
\text { (selection bias) }\end{array}$ & Unclear risk & Not described. \\
\hline $\begin{array}{l}\text { Blinding of } \\
\text { participants and } \\
\text { personnel }\end{array}$ & Low risk & $\begin{array}{l}\text { 24-week double-blind period, (we excluded the } 24 \text {-week } \\
\text { extension: only site- and patient-blind period). }\end{array}$ \\
\hline
\end{tabular}




\begin{tabular}{|c|c|c|}
\hline (performance bias) & & \\
\hline $\begin{array}{l}\text { Blinding of } \\
\text { outcome } \\
\text { assessment } \\
\text { (detection bias) }\end{array}$ & Low risk & $\begin{array}{l}\text { 24-week double-blind period, (we excluded the } 24 \text {-week } \\
\text { extension: only site- and patient-blind period). }\end{array}$ \\
\hline $\begin{array}{l}\text { Incomplete } \\
\text { outcome data } \\
\text { (attrition bias) }\end{array}$ & Low risk & $\begin{array}{l}411 / 451 \text { completed. Efficacy data were analyzed with a } \\
\text { full analysis set (FAS) that included all randomized } \\
\text { individuals who took at least one dose of double-blind } \\
\text { study medication, had a non-missing baseline value and } \\
\geq 1 \text { post-baseline efficacy value for } \geq 1 \text { efficacy variable. } \\
\text { The safety set comprised patients who took } \geq 1 \text { dose of } \\
\text { double-blind study medication. All drop outs and losses to } \\
\text { follow up accounted for. (Minor discrepancy between } \\
\text { paper } n=432 \text { randomized and supplementary data } n=451 \text { ) }\end{array}$ \\
\hline $\begin{array}{l}\text { Selective reporting } \\
\text { (reporting bias) }\end{array}$ & Low risk & All major clinically relevant outcomes were reported \\
\hline Other bias & Unclear risk & $\begin{array}{l}\text { This study was sponsored by Bristol-Myers Squibb and } \\
\text { AstraZeneca. Medical writing assistance was funded by } \\
\text { Bristol-Myers Squibb and AstraZeneca. }\end{array}$ \\
\hline
\end{tabular}

\section{Ji 2014}

\begin{tabular}{||l|l|}
\hline Methods & $\begin{array}{l}\text { l4- week randomized, double-blind, placebo-controlled, parallel } \\
\text { group, phase III study at } 40 \text { sites ( } 26 \text { in China, } 5 \text { each in Korea and } \\
\text { Taiwan, and } 4 \text { in India) }\end{array}$ \\
\hline \hline Participants & $\begin{array}{l}393 \text { initially randomized: placebo } \mathrm{n}=132 ; \text { dapagliflozin } 5 \mathrm{mg} \mathrm{n}=128 ; \\
\text { dapagliflozin } 10 \mathrm{mg} \mathrm{n}=133\end{array}$ \\
\hline Interventions & $\begin{array}{l}\text { Patients randomized to Dapagliflozin } 5 \mathrm{mg} \text { or } 10 \mathrm{mg} \text { or placebo once } \\
\text { daily for } 24 \text { weeks taken orally once per day before the first meal } \\
\text { of the day. } \\
\text { Six weeks lead-in on diet and exercise. } \\
\text { Rescue medication permitted (metformin (500mg daily, titrated to } \\
\text { 2000 mg if necessary) } \\
\text { Add on: none }\end{array}$ \\
\hline Outcomes & \begin{tabular}{l} 
Primary outcomes of the trial \\
\hline
\end{tabular} \\
\hline
\end{tabular}




\begin{tabular}{|c|c|}
\hline & $\begin{array}{l}\text { mean change in HbA1c level at week } 24 \text { dapagliflozin vs placebo } \\
\text { using the last-observation carried forward (LOCF) } \\
\text { Secondary outcomes of the trial } \\
\text { change from baseline in FPG } \\
\text { change from baseline } \\
\text { in 2-hourPPG (after a liquid meal challenge) } \\
\text { change from baseline in total weight } \\
\text { proportion of patients achieving a therapeutic glycemic response, } \\
\text { defined as HbA1c levels }<7.0 \% \text { (53mmol/mol), } \\
\text { change from base- line in } \beta \text { cell function and insulin resistance } \\
\text { (HOMA-2), } \\
\text { waist circumference } \\
\text { lipids (total cholesterol, LDL-C,HDL-C, and fasting triglyceride) } \\
\geq 3 \% \text { or } \geq 5 \% \text { reduction } \\
\text { spot fasting urinary glucose to creatinine ratio } \\
\text { Adverse events and safety assessments } \\
\text { discontinuations due to AEs, } \\
\text { laboratory tests } \\
\text { vital signs, } \\
\text { hypoglycemia, } \\
\text { genital infection and UTI, }\end{array}$ \\
\hline Inclusion criteria & $\begin{array}{l}\text { men and women }>18 \text { years, } \\
\text { type } 2 \text { diabetes, } \\
\text { drug naive, Including Chinese herbal medicines } \\
\mathrm{HbA} 1 \mathrm{c} \geq 7.5 \leq 10.5 \% \text { at enrolment } \\
\mathrm{C} \text {-peptide level } \geq 1.0 \mathrm{ng} / \mathrm{mL}(0.34 \mathrm{nmol} / \mathrm{L}) \\
\mathrm{BMI}<45 \mathrm{~kg} / \mathrm{m} 2 \text {. }\end{array}$ \\
\hline Exclusion criteria & $\begin{array}{l}\text { diabetes insipidus } \\
\text { symptoms of poorly controlled diabetes including marked polyuria } \\
\text { and polydipsia with }>10 \% \text { weight loss } \\
\text { diabetic ketoacidosis } \\
\text { hyper osmolar nonketotic coma } \\
\text { bone fractures (osteoporosis) } \\
\text { severe uncontrolled hypertension } \\
\text { myocardial infarction } \\
\text { cardiac surgery bypass surgery } \\
\text { unstable angina } \\
\text { congestive heart failure } \\
\text { transient ischemic attack or significant cerebrovascular disease } \\
\text { arrhythmia }\end{array}$ \\
\hline
\end{tabular}




\begin{tabular}{||l|l||}
\hline \hline renal disease \\
congenital renal glycosuria \\
Significant hepatic or hepatobiliary disease \\
history of hepatotoxicity \\
hemoglobinopathy, \\
donation of blood or blood products to a blood bank, blood \\
transfusion, \\
malignancy \\
immunocompromised, HIV \\
allergies to study medications or metformin \\
any antidiabetic therapy, including Chinese traditional medicine, \\
systemic corticosteroid therapy, \\
bariatric surgery \\
administration of sibutramine, phentermine, orlistat, rimonabant, \\
benzphetamine, diethylpropion, methamphetamine, \\
and/orphendimetrazine within 30daysofenrollmentvisit \\
at risk for dehydration or volume depletion \\
alcohol or other drug \\
previous participation in a clinical trial with dapagliflozin \\
and/oranyotherSGLT2-inhibitors \\
other investigational drug within 30 days \\
unstable or serious vascular, renal, hepatic, hematologic, \\
oncologic, endocrine, psychiatric, or rheumatic diseases \\
severe hyper triglyceridemia
\end{tabular}

\section{Risk of bias table}

\begin{tabular}{||l|l|l|}
\hline Bias & $\begin{array}{l}\text { Authors' } \\
\text { judgement }\end{array}$ & Support for judgement \\
\hline $\begin{array}{l}\text { Random sequence } \\
\text { generation (selection } \\
\text { bias) }\end{array}$ & Low risk & $\begin{array}{l}\text { Randomized sequentially by using a random numbers } \\
\text { in a 1:1:1 ratio, stratified according to site, Using } \\
\text { interactive voice response system in a blinded manner } \\
\text { to one of three treatment groups, stratified according } \\
\text { to site. }\end{array}$ \\
\hline $\begin{array}{l}\text { Allocation } \\
\text { concealment } \\
\text { (selection bias) }\end{array}$ & Unclear risk & $\begin{array}{l}\text { Not stated, but implied by interactive voice response } \\
\text { system for randomization. }\end{array}$ \\
\hline $\begin{array}{l}\text { Blinding of } \\
\text { participants and }\end{array}$ & Low risk & Double-blind. \\
\hline
\end{tabular}




\begin{tabular}{|l|l||l||}
\hline $\begin{array}{l}\text { personnel } \\
\text { (performance bias) }\end{array}$ & & \\
\hline $\begin{array}{l}\text { Blinding of outcome } \\
\text { assessment } \\
\text { (detection bias) }\end{array}$ & Low risk & Double-blind. \\
\hline $\begin{array}{l}\text { Incomplete outcome } \\
\text { data (attrition bias) }\end{array}$ & Low risk & $\begin{array}{l}\text { 343/393 completed. The last observation carried } \\
\text { forward method to impute missing observations, } \\
\text { excluding data asked the rescue therapy was used. All } \\
\text { drop outs and losses to follow-up accounted for. }\end{array}$ \\
\hline $\begin{array}{l}\text { Selective reporting } \\
\text { (reporting bias) }\end{array}$ & Low risk & $\begin{array}{l}\text { All the prespecified clinically relevant outcomes } \\
\text { reported. }\end{array}$ \\
\hline Other bias & Unclear risk & $\begin{array}{l}\text { This study was funded by Bristol-Myers Squibb and } \\
\text { Astrazeneca. Editorial and writing assistance was } \\
\text { funded by Bristol-Myers Squibb and Astrazeneca. Some } \\
\text { of the trialists are employees of Bristol-Myers Squibb. }\end{array}$ \\
\hline \hline
\end{tabular}

\section{Kadowaki 2014}

\begin{tabular}{|c|c|}
\hline Methods & $\begin{array}{l}\text { 12-week, randomized, double-blind, placebo-controlled, parallel- } \\
\text { group, dose-finding phase II trial, in } 32 \text { centers in Japan. }\end{array}$ \\
\hline Participants & $\begin{array}{l}547 \text { initially randomized: empagliflozin } 5 m g n=10,10 m g n=110 \text {, } \\
25 m g n=109,50 m g n=110 \text {, placebo }(n=109)\end{array}$ \\
\hline Interventions & $\begin{array}{l}\text { Randomized (1:1:1:1:1) to receive once-daily oral empagliflozin } 5 \text {, } \\
\text { 10, } 25,50 \mathrm{mg} \text {, versus placebo as monotherapy for } 12 \text { weeks (oral } \\
\text { dose). } \\
\text { Add on: diet and exercise counselling }\end{array}$ \\
\hline Outcomes & $\begin{array}{l}\text { At week } 12 . \\
\text { Primary outcomes of the trial } \\
\text { change from baseline in } \mathrm{HbA} 1 \mathrm{c} \text { at week } 12 . \\
\text { Secondary outcomes of the trial } \\
\text { percentage of patients with } \mathrm{HbA} 1 \mathrm{c} \geq 7.0 \% \text { at baseline who achieved } \\
\mathrm{HbA} 1 \mathrm{c}<7.0 \% \\
\text { change from baseline in fasting plasma glucose (FPG) } \\
\text { changes from baseline in body weight }\end{array}$ \\
\hline
\end{tabular}




\begin{tabular}{|c|c|}
\hline & $\begin{array}{l}\text { waist circumference } \\
\text { systolic (SBP) and diastolic (DBP) blood pressure } \\
\text { percentage of patients with }>5 \% \text { reduction in body weight } \\
\text { percentage of patients who had uncontrolled blood pressure at } \\
\text { baseline who had controlled blood pressure (SBP }<130 \mathrm{mmHg} \text { and } \\
\text { DBP }<80 \mathrm{mmHg} \text { ) } \\
\text { changes from baseline in HbA1c at week } 12 \text { were analyzed in } \\
\text { subgroups of patients with baseline HbA1c }<8.0 \% \text { and } \geq 8.0 \% \text {. } \\
\text { Adverse events and safety assessments } \\
\text { clinical laboratory tests, } \\
\text { vital signs, } \\
\text { 12-lead electrocardiogram, } \\
\text { physical examination, } \\
\text { use of rescue therapy } \\
\text { confirmed hypoglycemic AEs (plasma glucose } \leq 70 \mathrm{mg} / \mathrm{dL} \text { ( } \leq 3.9 \\
\text { mmol/L) and/or assistance required } \\
\text { urinary tract infection (UTI) } \\
\text { genital infection } \\
\text { volume depletion }\end{array}$ \\
\hline Inclusion criteria & $\begin{array}{l}\text { men and women } \\
\text { aged } \geq 20 \text { and } \leq 80 \text { years } \\
\text { T2DM } \\
\mathrm{BMI} \geq 18 \text { and } \leq 40 \mathrm{~kg} / \mathrm{m}^{2} \\
\text { on a diet and exercise regimen } \\
\text { drug-naive (no antidiabetes agents for } \geq 10 \text { weeks prior to consent) } \\
\text { HbA1c } \geq 7.0 \% \text { and } \leq 10.0 \% \text { at screening, } \\
\text { receiving } 1 \text { oral antidiabetes agent other than thiazolidinedione, } \\
\text { glucagon-like peptide analogues or insulin (unchanged for } \geq 10 \\
\text { weeks prior to consent) }\end{array}$ \\
\hline Exclusion criteria & $\begin{array}{l}\text { uncontrolled hyperglycemia (plasma glucose }(240 \mathrm{mg} / \mathrm{dL} \text { ( } 13.3 \\
\text { mmol/L) after an overnight fast during the washout/placebo run-in } \\
\text { period, confirmed by a second measurement), } \\
\text { moderate or severe renal impairment (eGFR) } \backslash 60 \mathrm{~mL} / \mathrm{min} / 1.73 \mathrm{~m}^{2} \\
\text { using the Japanese GFR estimation } \\
\text { liver disease (serum alanine aminotransferase, aspartate } \\
\text { aminotransferase, or alkaline phosphatase }>3 \times \text { upper limit of } \\
\text { normal) } \\
\text { acute coronary syndrome } \\
\text { a stroke or transient ischemic attack within } 12 \text { weeks of consent } \\
\text { anti-obesity drugs within } 12 \text { weeks of consent } \\
\text { bariatric surgery within } 2 \text { years, systemic steroids } \\
\text { change in the dose of thyroid hormones }\end{array}$ \\
\hline
\end{tabular}




\begin{tabular}{||l||l||}
\hline \hline Notes & $\begin{array}{l}\text { uncontrolled endocrine disorder other than T2DM } \\
\text { women of child-bearing potential were required to use adequate } \\
\text { contraception }\end{array}$ \\
\hline $\begin{array}{ll}\text { ClinicalTrials.gov identifier NCT01193218. } \\
\text { There is a 40-week randomized extension period (EMPA Kadowaki } \\
\text { 2015), in which patients who received empagliflozin } 10 \text { or } 25 \mathrm{mg} \text { in } \\
\text { the first 12-week treatment period continued this treatment, and } \\
\text { patients treated with placebo, empagliflozin } 5 \text { or } 50 \text { mg in the first } \\
12 \text {-week treatment period were switched to empagliflozin } 10 \text { or } 25 \\
\text { mg. The results from this extension period are not included in our } \\
\text { analyses. }\end{array}$ \\
\hline
\end{tabular}

Risk of bias table

\begin{tabular}{|c|c|c|}
\hline Bias & $\begin{array}{l}\text { Authors' } \\
\text { judgement }\end{array}$ & Support for judgement \\
\hline $\begin{array}{l}\text { Random sequence } \\
\text { generation (selection } \\
\text { bias) }\end{array}$ & Low risk & $\begin{array}{l}\text { Computer-generated random sequence and an } \\
\text { interactive web response system, stratified by } \\
\text { previous antidiabetes medication (sulfonylurea, non- } \\
\text { sulfonylurea, none), HbA1c levels and renal function. }\end{array}$ \\
\hline \begin{tabular}{|l|} 
Allocation \\
concealment (selection \\
bias)
\end{tabular} & Unclear risk & $\begin{array}{l}\text { Not described in detail but implied in the computer- } \\
\text { generated random sequence. }\end{array}$ \\
\hline \begin{tabular}{|l|} 
Blinding of participants \\
and personnel \\
(performance bias)
\end{tabular} & Low risk & Double-blind. \\
\hline $\begin{array}{l}\text { Blinding of outcome } \\
\text { assessment (detection } \\
\text { bias) }\end{array}$ & Low risk & Double-blind. \\
\hline $\begin{array}{l}\text { Incomplete outcome } \\
\text { data (attrition bias) }\end{array}$ & Low risk & $\begin{array}{l}\text { 528/547 completed. All drops outs and losses to } \\
\text { follow up accounted for. }\end{array}$ \\
\hline $\begin{array}{l}\text { Selective reporting } \\
\text { (reporting bias) }\end{array}$ & Low risk & $\begin{array}{l}\text { All clinical relevant outcomes are defined and } \\
\text { reported. }\end{array}$ \\
\hline Other bias & Unclear risk & $\begin{array}{l}\text { This study was funded by Boehringer Ingelheim, } \\
\text { Ingelheim, Germany and Eli Lilly, Indianapolis, USA. } \\
\text { Page processing charges for this manuscript were }\end{array}$ \\
\hline
\end{tabular}




\begin{tabular}{||l|l|}
\hline \hline & $\begin{array}{l}\text { Covered by Boehringer } \\
\text { Ingelheim, Ingelheim, Germany. Medical writing } \\
\text { assistance was funded by Boehringer Ingelheim. Some } \\
\text { of the trialists are employees of Boehringer } \\
\text { Ingelheim. }\end{array}$ \\
\hline
\end{tabular}

Kaku 2013

\begin{tabular}{|c|c|}
\hline Methods & $\begin{array}{l}\text { 12-week multicenter, randomized, five-arm, parallel-group, double- } \\
\text { blind, placebo-controlled study conducted at } 26 \text { centers in Japan. }\end{array}$ \\
\hline Participants & $\begin{array}{l}279 \text { initially randomized: placebo } n=54 \text {; or dapagliflozin ( } 1 \mathrm{mg} n=59 \text {; } \\
2.5 \mathrm{mg} n=56 ; 5 \mathrm{mg} n=58 ; 10 \mathrm{mg} n=52) \text {. }\end{array}$ \\
\hline Interventions & $\begin{array}{l}\text { Placebo or dapagliflozin }(1,2.5,5 \text { or } 10 \mathrm{mg}) \text { daily oral dose. } \\
\text { Add on: none. }\end{array}$ \\
\hline Outcomes & $\begin{array}{l}\text { Primary outcomes of the trial } \\
\text { change in HbA1c from baseline to week } 12 . \\
\text { Secondary outcomes of the trial } \\
\text { change from baseline in FPG at week 12, } \\
\text { proportion of patients achieving HbA1c <7.0\% at week 12, } \\
\text { proportion of patients achieving a therapeutic response (>0.5\% } \\
\text { placebo-corrected reduction in HbA1c or <7.0\% HbA1c), } \\
\text { change from baseline in body weight, } \\
\text { area under the curve from } 0 \text { to } 180 \text { min (AUCO-180 min) for } \\
\text { postprandial glucose; urinary glucose excretion (UGE) and } \\
\text { UGE/creatinine ratio; fasting insulin, } \\
\text { C-peptide response to an oral glucose tolerance test (OGTT), } \\
\beta \text {-cell function (HOMA-2), } \\
\text { insulin resistance (as measured by HOMA-2). } \\
\text { Adverse events and safety assessments } \\
\text { adverse events (AEs), } \\
\text { laboratory variables, } \\
\text { physical examination, } \\
\text { electrocardiogram, } \\
\text { vital signs, } \\
\text { urinary infections } \\
\text { genital infections }\end{array}$ \\
\hline
\end{tabular}




\begin{tabular}{|c|c|}
\hline & hypoglycemic and hyperglycemic events. \\
\hline Inclusion criteria & 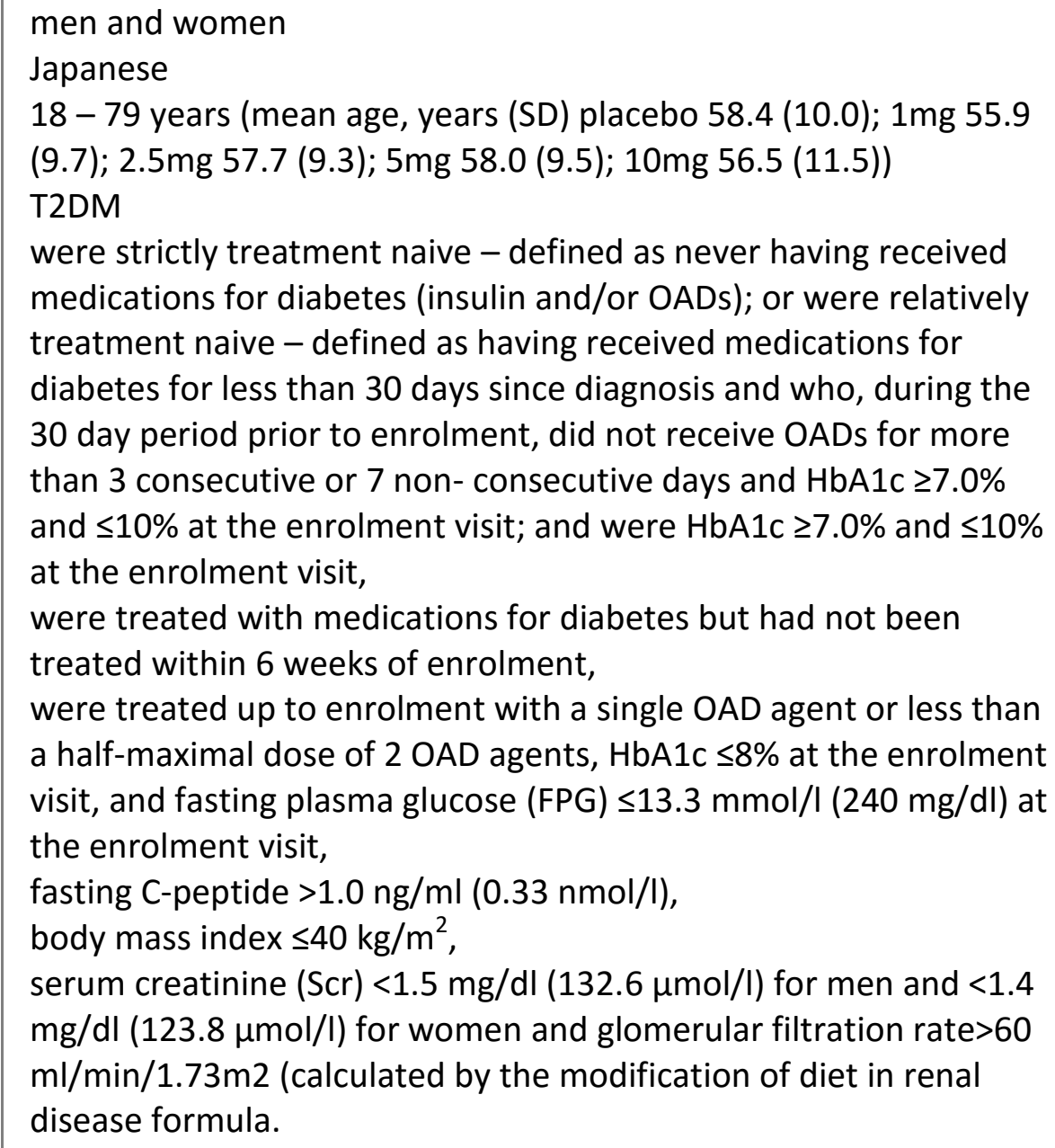 \\
\hline Exclusion criteria & $\begin{array}{l}\text { chronic insulin therapy within } 30 \text { days before the enrolment visit } \\
\text { (previous acute ( } \leq 2 \text { weeks) intermittent use of insulin was allowed, } \\
\text { as long as the last dose of insulin was administered more than } 2 \\
\text { weeks before the enrolment visit or for the treatment of } \\
\text { gestational diabetes), FPG }>13.3 \mathrm{mmol} / / \text { ( } 240 \mathrm{mg} / \mathrm{dl} \text { ) at weeks }-10,- \\
6,-4 \text { or }-1 \text { for patients treated with OADs up to enrolment, } \\
\text { cardiovascular events, } \\
\text { unstable renal disease, } \\
\text { retinopathy, } \\
\text { hepatic disease, } \\
\text { hematologic disease. }\end{array}$ \\
\hline Notes & ClinicalTrials.gov identifier: NCT00972244 \\
\hline
\end{tabular}

\section{Risk of bias table}




\begin{tabular}{|c|c|c|}
\hline Bias & $\begin{array}{l}\text { Authors' } \\
\text { judgement }\end{array}$ & Support for judgement \\
\hline $\begin{array}{l}\text { Random sequence } \\
\text { generation } \\
\text { (selection bias) }\end{array}$ & Low risk & $\begin{array}{l}\text { A computer-generated randomization list was used, based } \\
\text { on a permuted block method via an interactive voice } \\
\text { response system. A computer-generated randomization } \\
\text { list was used, based on a permuted block method, to } \\
\text { allocate patients to placebo or dapagliflozin }(1,2.5,5 \text { or } \\
10 \mathrm{mg}) \text { daily. }\end{array}$ \\
\hline $\begin{array}{l}\text { Allocation } \\
\text { concealment } \\
\text { (selection bias) }\end{array}$ & Unclear risk & $\begin{array}{l}\text { Not stated, but implied as patients were randomized via } \\
\text { an interactive voice response system. }\end{array}$ \\
\hline $\begin{array}{l}\text { Blinding of } \\
\text { participants and } \\
\text { personnel } \\
\text { (performance bias) }\end{array}$ & Low risk & Described as double-blind. \\
\hline $\begin{array}{l}\text { Blinding of } \\
\text { outcome } \\
\text { assessment } \\
\text { (detection bias) }\end{array}$ & Low risk & Described as double-blind. \\
\hline $\begin{array}{l}\text { Incomplete } \\
\text { outcome data } \\
\text { (attrition bias) }\end{array}$ & Low risk & $\begin{array}{l}258 / 279 \text { completed. Analysis of efficacy variables was } \\
\text { made using the full analysis set (FAS). The FAS included all } \\
\text { randomized patients who received at least one dose of } \\
\text { study medication, and who had a non-missing baseline } \\
\text { value and at least one post-baseline efficacy value for at } \\
\text { least one efficacy variable. The safety analysis set } \\
\text { included all randomized patients who received at least } \\
\text { one dose of study medication. All drop outs and losses to } \\
\text { follow up accounted for. }\end{array}$ \\
\hline $\begin{array}{l}\text { Selective reporting } \\
\text { (reporting bias) }\end{array}$ & Low risk & All clinically relevant outcomes were reported \\
\hline Other bias & Unclear risk & $\begin{array}{l}\text { Sponsored by Bristol-Myers Squibb and AstraZeneca. } \\
\text { Medical writing assistance funded by Bristol-Myers } \\
\text { Squibb and AstraZeneca. }\end{array}$ \\
\hline
\end{tabular}

Kaku 2014

Methods 24-week multicenter, randomized, five-arm, parallel-group, doubleblind, placebo-controlled study conducted in Japan. 


\begin{tabular}{|c|c|}
\hline Participants & 261 initially randomized: placebo $n=87 ; 5, g n=86 ; 10 m g n=88$ \\
\hline Interventions & $\begin{array}{l}\text { Placebo or dapagliflozin (5 or } 10 \mathrm{mg} \text { ) once daily for } 24 \text { weeks } \\
\text { Add on: not stated }\end{array}$ \\
\hline Outcomes & $\begin{array}{l}\text { Primary outcomes of the trial } \\
\text { change in HbA1c from baseline to week } 12 \text {. } \\
\text { Secondary outcomes of the trial } \\
\text { change from baseline to week } 24 \text { in total body weight in patients } \\
\text { with baseline body mass index (BMI) } \geq 25 \mathrm{~kg} / \mathrm{m} 2 \text {, } \\
\text { fasting insulin and C-peptide levels, } \\
\text { seated systolic blood pressure (SBP) overall and in patients with } \\
\text { baseline seated SBP } \geq 130 \mathrm{mmHg}, \\
\text { fasting lipids (total cholesterol, low-density lipoprotein cholesterol, } \\
\text { high-density lipoprotein cholesterol, free fatty acid and triglyceride } \\
\text { levels), } \\
\text { proportion of patients achieving a therapeutic glycemic response } \\
\text { (defined as HbA1c }<7 \% \text { ) after } 24 \text { weeks in patients with baseline } \\
\text { HbA1c } \geq 7 \% \text {, } \\
\text { proportion of patients discontinued for lack of efficacy or rescued } \\
\text { for failing to maintain FPG below prespecified rescue criteria after } \\
24 \text { weeks. } \\
\text { Adverse events and safety assessments } \\
\text { reported adverse events (AEs), } \\
\text { laboratory values, } \\
\text { ECG, heart rate, } \\
\text { blood pressure, } \\
\text { hypoglycemic events, } \\
\text { calculated creatinine clearance, eGFR, } \\
\text { physical examination findings. }\end{array}$ \\
\hline Inclusion criteria & $\begin{array}{l}\geq 20 \text { years } \\
\text { T2DM } \\
\text { drug-naive (never received medical treatment for diabetes or } \\
\text { received treatment for }<30 \text { days after diagnosis, and during the } 30 \text { - } \\
\text { day period before screening did not receive oral antidiabetic } \\
\text { agents for }>3 \text { consecutive or }>7 \text { non-consecutive days, or were } \\
\text { previously treated for diabetes but not within } 6 \text { weeks of } \\
\text { enrolment), } \\
\text { or receiving ongoing treatment for diabetes within } 6 \text { weeks of } \\
\text { enrolment (not drug-naive). }\end{array}$ \\
\hline
\end{tabular}




\begin{tabular}{|c|c|}
\hline & $\begin{array}{l}\mathrm{HbA} 1 \mathrm{c} \text { values } \geq 6.5 \text { and } \leq 10 \% \text { for patients defined as drug-naive, } \\
\text { and } \mathrm{HbA} 1 \mathrm{c} \text { values } \leq 8 \% \text { for patients with ongoing treatment. } \\
1 \text { week before randomization, } \mathrm{HbA} 1 \mathrm{c} \geq 6.5 \text { and } \leq 10 \% \\
\text { inadequately controlled by diet and exercise. }\end{array}$ \\
\hline Exclusion criteria & $\begin{array}{l}\text { type } 1 \text { diabetes or FPG }>240 \mathrm{mg} / \mathrm{dl}(13.3 \mathrm{mmol} / \mathrm{I}) \\
\text { pregnant or breastfeeding women, } \\
\text { creatinine kinase }>3 \times \text { upper limit of normal (ULN), } \\
\text { eGFR }<45 \mathrm{ml} / \mathrm{min} \text {, } \\
\text { serum } \mathrm{creatinine} \text { value of }>1.5 \mathrm{mg} / \mathrm{dl}(>133 \mu \mathrm{mol} / \mathrm{I}) \text { for men and } \\
>1.4 \mathrm{mg} / \mathrm{dl}(>124 \mu \mathrm{mol} / \mathrm{I}) \text { for women, } \\
\text { severe hepatic insufficiency and/or significant abnormal liver } \\
\text { function (aspartate aminotransferase }>3 \times \mathrm{ULN} \text { and/or alanine } \\
\text { aminotransferase }>3 \times \text { ULN), } \\
\text { New York Heart Association class IV congestive heart failure; } \\
\text { unstable or acute congestive heart failure, } \\
\text { treatment with thiazolidinediones }<6 \text { months before enrolment. }\end{array}$ \\
\hline Notes & No ClinicalTrials.gov identifier specified. \\
\hline
\end{tabular}

\section{Risk of bias table}

\begin{tabular}{|c|c|c|}
\hline Bias & \begin{tabular}{|l} 
Authors' \\
judgement
\end{tabular} & Support for judgement \\
\hline $\begin{array}{l}\text { Random sequence } \\
\text { generation } \\
\text { (selection bias) }\end{array}$ & Unclear risk & Not described, described as randomized. \\
\hline $\begin{array}{l}\text { Allocation } \\
\text { concealment } \\
\text { (selection bias) }\end{array}$ & Unclear risk & $\begin{array}{l}\text { Not described, described as randomized no details of } \\
\text { allocation concealment procedure. }\end{array}$ \\
\hline $\begin{array}{l}\text { Blinding of } \\
\text { participants and } \\
\text { personnel } \\
\text { (performance bias) }\end{array}$ & Low risk & Described as double-blind \\
\hline $\begin{array}{l}\text { Blinding of } \\
\text { outcome } \\
\text { assessment } \\
\text { (detection bias) }\end{array}$ & Low risk & Described as double-blind \\
\hline $\begin{array}{l}\text { Incomplete } \\
\text { outcome data }\end{array}$ & Low risk & 239/262 completed. \\
\hline
\end{tabular}




\begin{tabular}{|c|c|c|}
\hline (attrition bias) & & $\begin{array}{l}\text { Analysis of efficacy variables was made using the full } \\
\text { analysis set (FAS). The FAS included all randomized } \\
\text { patients who received at least one dose of study } \\
\text { medication, and who had a non-missing baseline value } \\
\text { and at least one post-baseline efficacy value for at least } \\
\text { one efficacy variable. The safety analysis set included all } \\
\text { randomized patients who received at least one dose of } \\
\text { study medication. FAS used last observation carried } \\
\text { forward (LOCF). All drop outs and losses to follow up } \\
\text { accounted for. } \\
\text { The safety set comprised patients who took } \geq 1 \text { dose of } \\
\text { double-blind study medication and who provided any } \\
\text { safety records. }\end{array}$ \\
\hline $\begin{array}{l}\text { Selective reporting } \\
\text { (reporting bias) }\end{array}$ & Low risk & All relevant outcomes seem to have been reported \\
\hline Other bias & Unclear risk & $\begin{array}{l}\text { The study was funded by AstraZeneca and Bristol-Myers } \\
\text { Squibb. Medical writing assistance was funded by } \\
\text { AstraZeneca and Bristol-Myers Squibb. }\end{array}$ \\
\hline
\end{tabular}

Kovacs 2014

\begin{tabular}{|c|c|}
\hline Methods & $\begin{array}{l}\text { 76-week, randomized, placebo-controlled trial, at } 69 \text { centers in eight } \\
\text { countries }\end{array}$ \\
\hline Participants & $\begin{array}{l}499 \text { initially randomized: empagliflozin } 10 \mathrm{mg} n=165 ; 25 \mathrm{mg} n=168 \text {; } \\
\text { placebo } n=165\end{array}$ \\
\hline Interventions & $\begin{array}{l}\text { Empagliflozin (10mg and } 25 \mathrm{mg} \text { once daily, oral dose) versus placebo } \\
\text { Add on: pioglitazone alone or pioglitazone plus metformin }\end{array}$ \\
\hline Outcomes & $\begin{array}{l}\text { Primary outcomes of the trial } \\
\text { change from baseline in } \mathrm{HbA} 1 \mathrm{c} \text { at week } 24 \text {. } \\
\text { Secondary outcomes of the trial } \\
\text { change from baseline in fasting plasma glucose (FPG) } \\
\text { change from baseline in body weight } \\
\mathrm{HbA} 1 \mathrm{c} \text { and FPG } \\
\text { percentage with HbA1c } \geq 7 \% \text { at baseline who achieved } \mathrm{HbA} 1 \mathrm{c}<7 \% \\
\text { at week } 24 \text {; } \\
\text { percentage of patients achieving a }>5 \% \text { reduction in body weight at }\end{array}$ \\
\hline
\end{tabular}




\begin{tabular}{|c|c|}
\hline & $\begin{array}{l}\text { week 24; } \\
\text { changes from baseline in waist circumference, } \\
\text { SBP and diastolic blood pressure (DBP) at week } 24 \\
\text { percentage of patients with uncontrolled blood pressure at } \\
\text { baseline who had controlled blood pressure (SBP <130mmHg and } \\
\text { DBP<80 mmHg) at week } 24 . \\
\text { change from baseline in HbA1c at week } 24 \text { was analyzed in } \\
\text { subgroups of patients receiving background therapy of pioglitazone } \\
\text { alone and pioglitazone plus metformin. Use of rescue therapy was } \\
\text { UGE was not measured in this study. } \\
\text { Adverse events and safety assessments } \\
\text { clinical laboratory tests } \\
\text { vital signs, } \\
12 \text {-lead electrocardiogram (ECG) } \\
\text { physical examination. } \\
\text { confirmed hypoglycemic AEs (plasma glucose } \leq 3.9 \text { mmol/l and/or } \\
\text { requiring assistance), } \\
\text { urinary tract infection (UTI) } \\
\text { genital infection } \\
\text { in patients receiving pioglitazone: a dedicated examination for } \\
\text { signs and symptoms of heart failure and edema was performed } 6 \\
\text { weeks after randomization, in addition to the standard physical } \\
\text { examination performed during the placebo run-in and at week } 24 \text {. }\end{array}$ \\
\hline Inclusion criteria & $\begin{array}{l}\text { men and women } \\
\text { aged } \geq 18 \text { years (and } \leq 65 \text { years in India) } \\
\text { T2DM } \\
\text { BMI } \leq 45 \mathrm{~kg} / \mathrm{m}^{2} \\
\mathrm{HbA} 1 \mathrm{c} \geq 7 \text { and } \leq 10 \% \text { at screening } \\
\text { on a diet and exercise regimen } \\
\text { for } \geq 12 \text { weeks prior to randomization, had been receiving } \\
\text { unchanged doses of pioglitazone monotherapy ( } \geq 30 \mathrm{mg} / \text { day, or the } \\
\text { maximum tolerated dose, or the maximum dose according to the } \\
\text { local label) or pioglitazone plus metformin ( } \geq 1500 \mathrm{mg} / \text { day, or } \\
\text { maximum tolerated dose or maximum dose according to the local } \\
\text { label). }\end{array}$ \\
\hline Exclusion criteria & $\begin{array}{l}\text { uncontrolled hyperglycemia (plasma glucose }>13.3 \mathrm{mmol} / \mathrm{l} \text { after an } \\
\text { overnight fast, confirmed by a second measurement), } \\
\text { severe renal impairment (estimated glomerular filtration rate } \\
\text { (eGFR) }<30 \mathrm{ml} / \mathrm{min} / 1.73 \mathrm{~m} 2 \text { using the Modification of Diet in Renal } \\
\text { Disease (MDRD) equation), } \\
\text { contraindication to pioglitazone and/or metformin according to the } \\
\text { local label, }\end{array}$ \\
\hline
\end{tabular}




\begin{tabular}{|c|c|}
\hline & $\begin{array}{l}\text { indication of liver disease (serum alanine aminotransferase, } \\
\text { aspartate aminotransferase or alkaline phosphatase }>3 \times \text { upper limit } \\
\text { of normal) } \\
\text { acute coronary syndrome, stroke or a transient ischemic attack } \\
\text { within } 3 \text { months of consent, } \\
\text { anti-obesity drugs } \\
\text { bariatric surgery in past } 2 \text { years } \\
\text { systemic steroids } \\
\text { change in the dose of thyroid hormones } \\
\text { uncontrolled endocrine disorder except T2D2 }\end{array}$ \\
\hline Notes & $\begin{array}{l}\text { ClinicalTrials.gov identifier: NCT01210001 } \\
\text { Empagliflozin vs placebo in patients on pioglitazone or pioglitazone } \\
\text { plus metformin. Empagliflozin } 10 \mathrm{mg} \text { arm not included as this low } \\
\text { dose was outside our inclusion criteria. } \\
\text { Double blind 52-week extension trial (i.e. } 76 \text { weeks in total) results } \\
\text { (Kovacs 2015) included in these analyses. } \\
\text { A non-randomized extension (NCT01289990) was also conducted; } \\
\text { these data are not included in our analyses. } \\
\text { "A dedicated Phase } 3 \text { trial (NCT01370005) will provide further } \\
\text { information on the effects of empagliflozin on BP." }\end{array}$ \\
\hline
\end{tabular}

Risk of bias table

\begin{tabular}{||l||l|l||}
\hline Bias & $\begin{array}{l}\text { Authors' } \\
\text { judgement }\end{array}$ & Support for judgement \\
\hline $\begin{array}{l}\text { Random sequence } \\
\text { generation (selection } \\
\text { bias) }\end{array}$ & Low risk & $\begin{array}{l}\text { Computer-generated random sequence and an } \\
\text { interactive voice and web response system }\end{array}$ \\
\hline $\begin{array}{l}\text { Allocation concealment } \\
\text { (selection bias) }\end{array}$ & Unclear risk & $\begin{array}{l}\text { Not described in detail but random sequence was } \\
\text { computer-generated. }\end{array}$ \\
\hline $\begin{array}{l}\text { Blinding of participants } \\
\text { and personnel } \\
\text { (performance bias) }\end{array}$ & Low risk & Double blind placebo controlled. \\
\hline $\begin{array}{l}\text { Blinding of outcome } \\
\text { assessment (detection } \\
\text { bias) }\end{array}$ & Low risk & Double blind placebo controlled. \\
\hline
\end{tabular}




\begin{tabular}{|l|l|l||}
\hline $\begin{array}{l}\text { Incomplete outcome } \\
\text { data (attrition bias) }\end{array}$ & Low risk & $\begin{array}{l}\text { 457/499 initially randomized completed (all 499 } \\
\text { contributed data to the final analysis set FAS). Last } \\
\text { observation carried forward. All drops outs and } \\
\text { losses to follow up accounted for. }\end{array}$ \\
\hline $\begin{array}{l}\text { Selective reporting } \\
\text { (reporting bias) }\end{array}$ & Low risk & $\begin{array}{l}\text { All clinically relevant outcomes are defined and } \\
\text { reported. }\end{array}$ \\
\hline Other bias & Unclear risk & $\begin{array}{l}\text { This study was funded by Boehringer Ingelheim. } \\
\text { Medical writing assistance, supported financially by } \\
\text { Boehringer Ingelheim. }\end{array}$ \\
\hline
\end{tabular}

\section{Lambers Heerspink 2013}

\begin{tabular}{|c|c|}
\hline Methods & $\begin{array}{l}\text { 12-week randomized, placebo-controlled, double-blind, multicenter, } \\
\text { three-arm, parallel-group, study conducted in Canada, The } \\
\text { Netherlands and the USA. }\end{array}$ \\
\hline Participants & $\begin{array}{l}75 \text { initially randomized: placebo }(n=25) \text {; dapagliflozin }(n=24) ; \\
\text { hydrochlorothiazide }(n=26)\end{array}$ \\
\hline Interventions & $\begin{array}{l}\text { Eligible patients (who met the inclusion criteria and adhered to } \\
\text { study medication) were randomly assigned to dapagliflozin } 10 \\
\mathrm{mg} / \text { day, hydrochlorothiazide } 25 \mathrm{mg} / \text { day, or matched placebo in a } \\
\text { 1:1:1 ratio (oral dose) for } 12 \text { weeks. } \\
\text { Add on: metformin, } \mathrm{SU}\end{array}$ \\
\hline Outcomes & $\begin{array}{l}\text { Primary outcomes of the trial } \\
\text { The study was exploratory in nature and designed as a mechanism- } \\
\text { of-action study to assess the effects of dapagliflozin on renal } \\
\text { function, BP and circulating plasma volume, } \\
\text { 24-h ambulatory BP monitoring, } \\
\text { GFR was determined by plasma disappearance of iohexol, } \\
\text { plasma volume and red cell mass. } \\
\text { Secondary outcomes of the trial } \\
\text { blood pressure, body weight and plasma volume. } \\
\text { Adverse events and safety assessments } \\
\text { adverse events, } \\
\text { urinary tract infection, } \\
\text { genital infection, }\end{array}$ \\
\hline
\end{tabular}




\begin{tabular}{|c|c|}
\hline & $\begin{array}{l}\text { hypoglycemia, } \\
\text { other adverse events including dizziness, syncope, } \\
\text { discontinuation of study medication to adverse event. }\end{array}$ \\
\hline Inclusion criteria & $\begin{array}{l}\text { men and women } \\
\text { T2DM } \\
\text { aged between } 18 \text { and } 70 \text { years } \\
\text { inadequate glycemic control, defined as } \mathrm{HbA} 1 \mathrm{c} \geq 6.6 \% \text { and } \leq 9.5 \% \\
\text { receiving a stable dose of metformin and/or a sulfonylurea } \\
\text { derivative for at least } 4 \text { weeks prior to study entry. } \\
\text { C-peptide } \geq 0.27 \mathrm{nmol} / \mathrm{l} \text {, } \\
\text { eGFR }>60 \mathrm{ml} / \mathrm{min} / 1.73 \mathrm{~m} 2 \text { and }<150 \mathrm{ml} / \mathrm{min} / 1.73 \mathrm{~m}^{2} \text {, } \\
\text { urine albumin:creatinine ratio }<300 \mathrm{mg} / \mathrm{g} \text {, } \\
\text { body mass index } \leq 45.0 \mathrm{~kg} / \mathrm{m}^{2} \text {, } \\
\text { inadequate } \mathrm{BP} \text { control, defined as systolic blood pressure }(\mathrm{SBP}) \geq \\
130 \text { and }<165 \mathrm{mmHg} \text {, and/or diastolic } \mathrm{BP} \geq 80 \text { and }<105 \mathrm{mmHg} \text {. }\end{array}$ \\
\hline Exclusion criteria & $\begin{array}{l}\text { type } 1 \text { diabetes } \\
\text { cardiovascular disease within } 6 \text { months of study entry, } \\
\text { pregnant women } \\
\text { history of adverse reaction to radiocontrast dye, } \\
\text { allergy to or contraindication for thiazide diuretics. }\end{array}$ \\
\hline Notes & $\begin{array}{l}\text { Participants in the placebo group were slightly older than those in } \\
\text { the other two treatment groups. } \\
\text { ClinicalTrials.gov identifier: NСT00976495 }\end{array}$ \\
\hline
\end{tabular}

\section{Risk of bias table}

\begin{tabular}{|l|l|l|l||}
\hline Bias & $\begin{array}{l}\text { Authors' } \\
\text { judgement }\end{array}$ & Support for judgement \\
\hline $\begin{array}{l}\text { Random sequence } \\
\text { generation } \\
\text { (selection bias) }\end{array}$ & Low risk & $\begin{array}{l}\text { Study treatments were allocated using a central, } \\
\text { computer-based randomization procedure. }\end{array}$ \\
\hline $\begin{array}{l}\text { Allocation } \\
\text { concealment } \\
\text { (selection bias) }\end{array}$ & Unclear risk & $\begin{array}{l}\text { Not stated, but implied as study treatments were } \\
\text { allocated using a central, computer-based randomization } \\
\text { procedure. }\end{array}$ \\
\hline $\begin{array}{l}\text { Blinding of } \\
\text { participants and } \\
\text { personnel }\end{array}$ & Low risk & $\begin{array}{l}\text { Blinding of patients and investigators to study treatment } \\
\text { was achieved using a double-dummy technique. }\end{array}$ \\
\hline
\end{tabular}




\begin{tabular}{|c|c|c|}
\hline (performance bias) & & \\
\hline $\begin{array}{l}\text { Blinding of } \\
\text { outcome } \\
\text { assessment } \\
\text { (detection bias) }\end{array}$ & Low risk & $\begin{array}{l}\text { Not described if all outcome assessments were blinded } \\
\text { (e.g. coding of adverse events), but likely they were as } \\
\text { study describes the use of MedDRA terms and study } \\
\text { described as double blinded. }\end{array}$ \\
\hline $\begin{array}{l}\text { Incomplete } \\
\text { outcome data } \\
\text { (attrition bias) }\end{array}$ & Low risk & $\begin{array}{l}\text { 74/75 completed ( } 1 \text { withdrew consent). If no week } 12 \\
\text { measurement was available, the last available post- } \\
\text { baseline measurement was used for analysis of } 24-\mathrm{h} \text {, day- } \\
\text { time and night-time BP, and the last available post- } \\
\text { baseline measurement after week } 4 \text { was used for analysis } \\
\text { of GFR, plasma volume and red cell mass. }\end{array}$ \\
\hline $\begin{array}{l}\text { Selective reporting } \\
\text { (reporting bias) }\end{array}$ & Unclear risk & $\begin{array}{l}\text { Due to the exploratory nature of the study, no statistical } \\
\text { comparisons between treatment arms were made. } \\
\text { Median changes in } 24-h \text { ambulatory BP, plasma volume } \\
\text { and red cell mass are reported in the sub study because } \\
\text { of the small sample size of the sub study and the fact that } \\
\text { mean changes can be driven by a few extreme values. }\end{array}$ \\
\hline Other bias & Unclear risk & $\begin{array}{l}\text { The trial was sponsored by Bristol-Myers Squibb and } \\
\text { AstraZeneca. Some of the trialists are employees of } \\
\text { Bristol-Myers Squibb. }\end{array}$ \\
\hline
\end{tabular}

\section{Leiter 2014}

\begin{tabular}{|c|c|}
\hline Methods & $\begin{array}{l}\text { 52-week, multicenter, randomized, double-blind, age-stratified, } \\
\text { placebo-controlled study in } 173 \text { centers in } 10 \text { countries United } \\
\text { States, Canada, Australia, Chile, Argentina, and five European } \\
\text { countries. }\end{array}$ \\
\hline Participants & 964 initially randomized: placebo $n=483$; dapagliflozin $n=482$ \\
\hline Interventions & $\begin{array}{l}\text { Randomized } 1: 1 \text { to receive once-daily dapagliflozin } 10 \mathrm{mg} \text { or } \\
\text { matched placebo for } 24 \text { weeks. } \\
\text { participants reduced their average daily insulin dose by } 25 \% \text { at } \\
\text { randomization } \\
\text { rescue medication permitted and rescue criteria became stricter } \\
\text { during the course of the study, as follows: weeks } 0-4, F P G>270 \\
\mathrm{mg} / \mathrm{dL} \text {; weeks } 5-8, \mathrm{FPG}>240 \mathrm{mg} / \mathrm{dL} \text {; weeks } 9-24, \mathrm{FPG}>200 \mathrm{mg} / \mathrm{dL} \text {; } \\
\text { week } 25 \text { onward, HbA1c }>8.0 \% \text {. } \\
\text { antihypertensive rescue (choice of medication at investigator } \\
\text { discretion) was given after repeated confirmed measurements of }\end{array}$ \\
\hline
\end{tabular}


blood pressure.

Add on: pre-existing, stable background treatment of antidiabetic drugs, including insulin, i.e. participants continued study treatment and concomitant medications.

\section{Primary outcomes of the trial}

Glycemic efficacy at 24 weeks mean change in $\mathrm{HbA} 1 \mathrm{c}$ from baseline and proportion of participants achieving a three item outcome measure of combined clinical benefit: simultaneous $\mathrm{HbA} 1 \mathrm{c}$ decrease of $0.5 \%$ or greater, total body weight reduction of $3 \%$ or greater, and systolic BP (SBP) reduction of $3 \mathrm{mmHg}$ or more from baseline.

\section{Secondary outcomes of the trial}

mean percent change in total body weight (BW) from baseline to week 24 , the proportion of patients with a baseline body mass index $\geq 27 \mathrm{~kg} / \mathrm{m}^{2}$ achieving a reduction in BW of $\geq 5 \%$ at week 24 , seated systolic BP at weeks 8 and 24 , and seated systolic BP in patients with a baseline seated systolic $B P \geq 130 \mathrm{mmHg}$. mean change from baseline in diastolic BP overall and in patients with seated baseline systolic $B P \geq 130 \mathrm{mmHg}$ at weeks 8 and 24; mean change in seated systolic BP in patients who had baseline systolic $B P \geq 130 \mathrm{mmHg}$ at week 24; mean change in BW from baseline to week 24;

pulse change in hemoglobin $\mathrm{A} 1 \mathrm{c}(\mathrm{HbA} 1 \mathrm{c})$ in patients with baseline $\mathrm{HbA} 1 \mathrm{c}$ $\geq 8.0 \%$ and $\mathrm{HbA} 1 \mathrm{C} \geq 9.0 \%$ at week 24 ;

change in FPG at week 1 and week 24;

change in calculated average daily insulin dose in patients treated with insulin at baseline at week 24; change in plasma uric acid levels at week 24. proportion with seated systolic $B P<130 \mathrm{mmHg}$ at week 24 in the population with seated systolic $B P \geq 130 \mathrm{mmHg}$ at baseline; rescue for failing to maintain FPG below prespecified rescue criteria with dapagliflozin $10 \mathrm{mg}$ versus placebo at weeks $4,8,16$, and 24;

failure to maintain BP (systolic and diastolic) below prespecified rescue criteria with dapagliflozin $10 \mathrm{mg}$ versus placebo at weeks 8 , 16, and 24;

reduction from baseline of $3 \mathrm{mmHg}$ or more and $5 \mathrm{mmHg}$ or more in seated systolic BP at week 24;

reduction from baseline of $3 \mathrm{mmHg}$ or more and $5 \mathrm{mmHg}$ or more in seated systolic BP at week 24;

proportion of patients achieving a therapeutic response, defined as 


\begin{tabular}{|c|c|}
\hline & $\begin{array}{l}\text { a reduction in HbA1c of } 0.5 \% \text { or more, at week } 24 . \\
\text { Adverse events and safety assessments } \\
\text { urinary tract infection } \\
\text { genital infection } \\
\text { discontinuations due to AEs, } \\
\text { prespecified laboratory abnormalities } \\
\text { renal impairment and failure } \\
\text { changes in creatinine renal clearance and blood creatinine } \\
\text { kidney infection } \\
\text { hypotension, dehydration, or hypovolemia } \\
\text { syncope } \\
\text { circulatory collapse } \\
\text { increases in hematocrit } \\
\text { liver function tests } \\
\text { glomerular function }\end{array}$ \\
\hline Inclusion criteria & $\begin{array}{l}\text { men and women } \\
\text { older adults "Participants aged } 75 \text { and older ( } 7.7 \% \text { of total) were } \\
\text { distributed as follows: } 46 \text { participants in the dapagliflozin group } \\
(9.6 \%) \text { and } 28 \text { in the placebo group (5.8\%)" Mean age (SD) placebo } \\
63.6(7.0) \text {; dapagliflozin } 63.9 \text { (7.6) } \\
\text { T2DM (HbA1c 7.0-10.0\%) } \\
\text { preexisting cardiovascular disease (defined as prior documented } \\
\text { coronary heart disease, including history of myocardial infarction } \\
\text { or revascularization or coronary artery stenosis }>50 \% \text {, confirmed } \\
\text { with angiography or abnormal stress test imaging, compatible with } \\
\text { ischemias or prior myocardial infarction; or prior documented } \\
\text { stroke or transient ischemic attack; or (3) prior documented } \\
\text { peripheral artery disease treated with revascularization (excluding } \\
\text { amputation). }\end{array}$ \\
\hline Exclusion criteria & $\begin{array}{l}\text { type } 1 \text { diabetes mellitus, } \\
\text { use of rosiglitazone or three or more oral antihyperglycemic drugs, } \\
\text { symptoms of poorly controlled diabetes such as marked polyuria, } \\
\text { polydipsia, and/or }>10 \% \text { weight loss, } \\
\text { fasting plasma glucose (FPG) }>270 \mathrm{mg} / \mathrm{dL} \text {, } \\
\text { cardiovascular events within } 2 \text { months of enrolment, } \\
\text { New York Association class IV congestive heart failure, } \\
\text { unstable or acute congestive heart failure, } \\
\text { systolic blood pressure (BP) } \geq 160 \mathrm{mmHg} \text { and/or diastolic BP } \geq 100 \\
\text { mmHg at randomization, } \\
\text { calculated creatinine clearance }<60 \mathrm{~mL} / \mathrm{min} \text {; urine } \\
\text { albumin:creatinine ratio }>1,800 \mathrm{mg} / \mathrm{g} \text {, }\end{array}$ \\
\hline
\end{tabular}




\begin{tabular}{|l||l||}
\hline \hline Notes & history of unstable or rapidly progressing renal disease. \\
\hline \hline & $\begin{array}{l}\text { ClinicalTrials.gov identifier: NCT01042977 } \\
\text { "Participants aged } 75 \text { and older (7.7\% of total) were distributed as } \\
\text { follows: } 46 \text { participants in the dapagliflozin group (9.6\%) and } 28 \text { in } \\
\text { the placebo group (5.8\%)" } \\
\text { "Demographic and baseline characteristics of participants were well } \\
\text { balanced between treatments (able 1) and age strata" } \\
\text { Trial report includes } 28 \text { - and 52-week extension periods. The primary } \\
\text { efficacy analysis was conducted at week 24, with exploratory } \\
\text { efficacy outcomes at week 52. }\end{array}$ \\
\hline
\end{tabular}

\section{Risk of bias table}

\begin{tabular}{|c|c|c|}
\hline Bias & $\begin{array}{l}\text { Authors' } \\
\text { judgement }\end{array}$ & Support for judgement \\
\hline $\begin{array}{l}\text { Random sequence } \\
\text { generation } \\
\text { (selection bias) }\end{array}$ & Low risk & $\begin{array}{l}\text { Patients were assigned a unique enrolment number using } \\
\text { Interactive Web Response System (IWRS) or Interactive } \\
\text { Voice Response System (IVRS) at visit1 (enrolment visit). } \\
\text { Patients were randomized to the treatment groups using } \\
\text { the method of randomly permuted blocks with a block size } \\
\text { of four after evaluation for all inclusion/exclusion criteria. } \\
\text { Patients were stratified into one of the eightage-by-insulin } \\
\text { use-by-time from most recent qualifying cardiovascular } \\
\text { event strata according to age group (<65 years vs } \geq 65 \text { years } \\
\text { at enrolment), use of insulin (no vs. yes at randomization) } \\
\text { and time from most recent qualifying cardiovascular event } \\
\text { (more than } 1 \text { year vs } 1 \text { year or less (i.e., within } 12 \text { months) } \\
\text { before enrolment). Patients were given medication with } \\
\text { the bottle number allocated by the IWRS/IVRS. }\end{array}$ \\
\hline $\begin{array}{l}\text { Allocation } \\
\text { concealment } \\
\text { (selection bias) }\end{array}$ & Unclear risk & $\begin{array}{l}\text { Not stated, but implied by use of computerized system, as } \\
\text { described for randomization. }\end{array}$ \\
\hline $\begin{array}{l}\text { Blinding of } \\
\text { participants and } \\
\text { personnel } \\
\text { (performance bias) }\end{array}$ & Low risk & Double-blind. \\
\hline Blinding of & Low risk & Double-blind. \\
\hline
\end{tabular}




\begin{tabular}{|l||l||l||}
\hline $\begin{array}{l}\text { outcome } \\
\text { assessment } \\
\text { (detection bias) }\end{array}$ & & \\
\hline $\begin{array}{l}\text { Incomplete } \\
\text { outcome data } \\
\text { (attrition bias) }\end{array}$ & Unclear risk & $\begin{array}{l}870 / 964 \text { completed. All drop outs and losses to follow up } \\
\text { accounted for. Published report states that 962 comprised } \\
\text { the full analysis set. 965 started double-blind placebo } \\
\text { treatment in total (i.e. one more than the number } \\
\text { randomized) }\end{array}$ \\
\hline $\begin{array}{l}\text { Selective reporting } \\
\text { (reporting bias) }\end{array}$ & Low risk & All the prespecified clinically relevant outcomes reported. \\
\hline Other bias & Unclear risk & $\begin{array}{l}\text { Funded by AstraZeneca and Bristol-Myers Squibb. The } \\
\text { sponsors had no part in the study and design. AstraZeneca } \\
\text { and Bristol-Myers Squibb, provided editorial support. }\end{array}$ \\
\hline
\end{tabular}

Lewin 2015

\begin{tabular}{|c|c|}
\hline Methods & $\begin{array}{l}52 \text {-week phase } 3, \text { randomized, double-blind, parallel-group study in } \\
197 \text { centers in } 22 \text { countries. }\end{array}$ \\
\hline Participants & $\begin{array}{l}677 \text { initially randomized: empagliflozin } 25 \mathrm{mg} / \text { linagliptin } 5 \mathrm{mg}(\mathrm{n}= \\
137) \text {, empagliflozin } 10 \mathrm{mg} / \text { linagliptin } 5 \mathrm{mg}(\mathrm{n}=136) \text {, empagliflozin } \\
25 \mathrm{mg}(\mathrm{n}=135) \text {, empagliflozin } 10 \mathrm{mg}(\mathrm{n}=134) \text {, or } \\
\text { linagliptin } 5 \mathrm{mg}(\mathrm{n}=135)\end{array}$ \\
\hline Interventions & $\begin{array}{l}\text { Randomized (1:1:1:1:1) to receive empagliflozin } 25 \mathrm{mg} / \text { linagliptin } 5 \\
\text { mg, empagliflozin } 10 \mathrm{mg} / \text { linagliptin } 5 \mathrm{mg} \text {, empagliflozin } 25 \mathrm{mg} \text {, } \\
\text { empagliflozin } 10 \mathrm{mg} \text {, or linagliptin } 5 \mathrm{mg} \text { for } 52 \text { weeks. Once daily oral } \\
\text { dose. } \\
\text { Add on: diet and exercise. } \\
\text { Rescue medication permitted in case of hyperglycemia. }\end{array}$ \\
\hline Outcomes & $\begin{array}{l}\text { Primary outcomes of the trial } \\
\text { change from baseline in } \mathrm{HbA} 1 \mathrm{c} \text { at week } 24 \text {. } \\
\text { Secondary outcomes of the trial } \\
\text { change from baseline in FPG at week } 24 \text {, } \\
\text { change from baseline in weight at week } 24 \\
\text { proportion of subjects with baseline } \mathrm{HbA} 1 \mathrm{c} \geq 7 \% \text { ( } \$ 53 \mathrm{mmol} / \mathrm{mol}) \\
\text { who had } \mathrm{HbA} 1 \mathrm{c}<7 \%(<53 \mathrm{mmol} / \mathrm{mol}) \text { at week } 24 \text {. Exploratory end }\end{array}$ \\
\hline
\end{tabular}




\begin{tabular}{|c|c|}
\hline & $\begin{array}{l}\text { points were as follows: } \\
\text { changes from baseline in } \mathrm{HbA} 1 \mathrm{c} \text { at week } 24 \text { in subgroups with } \\
\mathrm{HbA} 1 \mathrm{c} \geq 8.5 \% \text { ( } \geq 69 \mathrm{mmol} / \mathrm{mol} \text { ) and }<8.5 \% \text { ( }<69 \mathrm{mmol} / \mathrm{mol} \text { ) at } \\
\text { baseline } \\
\text { changes from baseline in } \mathrm{HbA} 1 \mathrm{c}, \mathrm{FPG}, \\
\text { weight, } \\
\text { systolic BP (SBP), and diastolic BP (DBP) at week } 52 ; \\
\text { proportion of subjects with baseline } \mathrm{HbA} 1 \mathrm{c} \geq 7 \% \text { ( } \geq 53 \mathrm{mmol} / \mathrm{mol}) \\
\text { who had HbA1c }<7 \% \text { ( }<53 \mathrm{mmol} / \mathrm{mol} \text { ) at week } 52 \text {. } \\
\text { Adverse events and safety assessments } \\
\text { vital signs, } \\
\text { clinical laboratory parameters, } \\
\text { adverse events including all events with an onset after the first } \\
\text { dose and up to } 7 \text { days after the last dose of study medication. } \\
\text { confirmed hypoglycemic events } \\
\text { urinary tract infection (UTI), } \\
\text { genital infection, } \\
\text { volume depletion, } \\
\text { hypersensitivity reactions, } \\
\text { pancreatitis. }\end{array}$ \\
\hline Inclusion criteria & $\begin{array}{l}\text { men and women, } \\
\geq 18 \text { years, } \\
\text { T2DM, } \\
\mathrm{BMI} \leq 45 \mathrm{~kg} / \mathrm{m}^{2}, \\
\mathrm{HbA} 1 \mathrm{c}>7 \% \text { to } \leq 10.5 \% \text { ( }>53 \text { to }<91 \mathrm{mmol} / \mathrm{mol} \text { ) at screening despite } \\
\text { a diet and exercise regimen, } \\
\text { not received treatment with oral antidiabetes therapy, GLP-1 } \\
\text { analogue, or insulin for } \geq 12 \text { weeks prior to randomization. }\end{array}$ \\
\hline Exclusion criteria & $\begin{array}{l}\text { uncontrolled hyperglycemia (glucose level } .240 \mathrm{mg} / \mathrm{dL} \text { after an } \\
\text { overnight fast during the placebo run-in, } \\
\text { eGFR }>60 \mathrm{~mL} / \mathrm{min} / 1.73 \mathrm{~m} 2 \text { (using the MDRD equation) } \\
\text { acute coronary syndrome, stroke, or transient ischemic attack } \\
\text { within } 3 \text { months prior to consent; } \\
\text { bariatric surgery in the last } 2 \text { years } \\
\text { treatment with anti-obesity drugs within } 3 \text { months prior to } \\
\text { consent. }\end{array}$ \\
\hline Notes & $\begin{array}{l}\text { ClinicalTrials.gov identifier: NCT01422876 } \\
\text { Initial treatment was with empagliflozin for } 24 \text { weeks, extended to } \\
52 \text { weeks. }\end{array}$ \\
\hline
\end{tabular}


Risk of bias table

\begin{tabular}{|c|c|c|}
\hline Bias & $\begin{array}{l}\text { Authors' } \\
\text { judgement }\end{array}$ & Support for judgement \\
\hline $\begin{array}{l}\text { Random sequence } \\
\text { generation (selection } \\
\text { bias) }\end{array}$ & Unclear risk & $\begin{array}{l}\text { Randomized but not described fully in the published } \\
\text { report of the study. }\end{array}$ \\
\hline $\begin{array}{l}\text { Allocation concealment } \\
\text { (selection bias) }\end{array}$ & Unclear risk & Not described. \\
\hline $\begin{array}{l}\text { Blinding of participants } \\
\text { and personnel } \\
\text { (performance bias) }\end{array}$ & Low risk & Double-blind. \\
\hline $\begin{array}{l}\text { Blinding of outcome } \\
\text { assessment (detection } \\
\text { bias) }\end{array}$ & Low risk & Double-blind. \\
\hline $\begin{array}{l}\text { Incomplete outcome } \\
\text { data (attrition bias) }\end{array}$ & Low risk & $\begin{array}{l}\text { 572/677 subjects completed } 52 \text { weeks treatment. } \\
667 \text { comprised the analysis data set. All drops outs } \\
\text { and losses to follow up accounted for. }\end{array}$ \\
\hline $\begin{array}{l}\text { Selective reporting } \\
\text { (reporting bias) }\end{array}$ & Low risk & $\begin{array}{l}\text { All clinically relevant outcomes are defined and } \\
\text { reported. }\end{array}$ \\
\hline Other bias & Unclear risk & $\begin{array}{l}\text { This study was funded by Boehringer Ingelheim and } \\
\text { Eli Lilly and Company. The trialists has served on } \\
\text { scientific advisory boards for, or are employees of, } \\
\text { Boehringer Ingelheim } \\
\text { Medical writing assistance was supported financially } \\
\text { by Boehringer Ingelheim. }\end{array}$ \\
\hline
\end{tabular}

List 2009

\begin{tabular}{||l|l||}
\hline Methods & $\begin{array}{l}\text { 12-week, randomized, parallel-group, double-blind, placebo- } \\
\text { controlled study } 98 \text { clinical centers in the U.S., } 24 \text { in Canada, } 8 \text { in } \\
\text { Mexico, and } 3 \text { in Puerto Rico. }\end{array}$ \\
\hline Participants & $\begin{array}{l}389 \text { initially randomized: } 5 \mathrm{mg} \text { dapagliflozin } \mathrm{n}=58 ; 10 \mathrm{mg} \\
\text { dapagliflozin } n=47 ; 20 \mathrm{mg} \text { dapagliflozin } n=59 ; 50 \mathrm{mg} \text { dapagliflozin } \\
n=56 ; \text { metformin } n=56\end{array}$ \\
\hline Interventions & Randomly assigned equally to once-daily dapagliflozin $(2.5,5,10,20$, \\
\hline
\end{tabular}




\begin{tabular}{|c|c|}
\hline & $\begin{array}{l}\text { or } 50 \mathrm{mg} \text { ), metformin XR ( } 750 \mathrm{mg} \text { force-titrated at week } 2 \text { to } 1,500 \\
\mathrm{mg} \text { ) (therapeutic benchmark), or placebo. Daily for } \times 12 \text { weeks (oral } \\
\text { dose). } \\
\text { Add on: none. } \\
\text { Use of rescue medication, not described in the published study } \\
\text { report. }\end{array}$ \\
\hline Outcomes & $\begin{array}{l}\text { Primary outcomes of the trial } \\
\text { mean A1C change from baseline after } 12 \text { weeks. } \\
\text { Secondary outcomes of the trial } \\
\text { FPG change from baseline, } \\
\text { dose dependent trends in glycemic efficacy, proportion of patients } \\
\text { achieving A1C } \leq 7 \% \text {, } \\
\text { change in } 24-h \text { urinary glucose- to-creatinine ratio } \\
\text { total weight loss. } \\
\text { Adverse events and safety assessments } \\
\text { vital signs, } \\
\text { brief physical examination, } \\
\text { adverse event assessment at each visit. } \\
\text { complete physical examination and electrocardiograms } \\
\text { adverse events } \\
\text { safety topics of special interest were summarized by interest } \\
\text { categories. e.g. urinary tract infections }\end{array}$ \\
\hline Inclusion criteria & $\begin{array}{l}\text { men and women } \\
\text { aged } 18-79 \\
\text { T2DM } \\
\text { A1C } \geq 7 \% \text { and } \leq 10 \% \\
\text { fasting } C \text { peptide }>1.0 \mathrm{ng} / \mathrm{ml} \text {, } \\
\text { BMI } \leq 40 \mathrm{~kg} / \mathrm{m}^{2} \text {, } \\
\text { renal status: glomerular filtration rate }>60 \mathrm{ml} / \mathrm{min} \text { per } 1.73 \mathrm{~m}^{2} \text {, } \\
\text { serum creatinine }<1.5 \mathrm{mg} / \mathrm{dl}(\mathrm{men}) /<1.4 \mathrm{mg} / \mathrm{dl} \text { (women), and urine } \\
\text { microalbumin/ creatinine ratio } \leq 300 \mathrm{mg} / \mathrm{g} \text {. }\end{array}$ \\
\hline Exclusion criteria & No exclusion criteria were described in the published report. \\
\hline Notes & $\begin{array}{l}\text { ClinicalTrials.gov identifier: NCT00263276 } \\
\text { Three arms; dapagliflozin vs placebo, dapagliflozin vs. metformin. }\end{array}$ \\
\hline
\end{tabular}


Baseline demographics and disease characteristics were similar among all groups

Risk of bias table

\begin{tabular}{|c|c|c|}
\hline Bias & $\begin{array}{l}\text { Authors' } \\
\text { judgement }\end{array}$ & Support for judgement \\
\hline $\begin{array}{l}\text { Random sequence } \\
\text { generation (selection } \\
\text { bias) }\end{array}$ & Unclear risk & Not reported. \\
\hline $\begin{array}{l}\text { Allocation } \\
\text { concealment } \\
\text { (selection bias) }\end{array}$ & Unclear risk & Not reported. \\
\hline $\begin{array}{l}\text { Blinding of } \\
\text { participants and } \\
\text { personnel } \\
\text { (performance bias) }\end{array}$ & Low risk & Double blind. \\
\hline $\begin{array}{l}\text { Blinding of outcome } \\
\text { assessment } \\
\text { (detection bias) }\end{array}$ & Low risk & Double blind. \\
\hline $\begin{array}{l}\text { Incomplete outcome } \\
\text { data (attrition bias) }\end{array}$ & Low risk & $\begin{array}{l}\text { 348/389 } 348 \text { completed week } 12 \text {, and } 41 \text { discontinued. } \\
\text { Last observation carried forward. }\end{array}$ \\
\hline $\begin{array}{l}\text { Selective reporting } \\
\text { (reporting bias) }\end{array}$ & Unclear risk & $\begin{array}{l}\text { All clinical relevant outcomes are defined and } \\
\text { reported, however it is unclear which secondary or } \\
\text { exploratory outcomes were of interest as these were } \\
\text { not all described prospectively in the published report } \\
\text { of the study results. }\end{array}$ \\
\hline Other bias & Unclear risk & $\begin{array}{l}\text { This study was supported by Bristol-Myers Squibb and } \\
\text { Astra- Zeneca. Editorial support was funded by Bristol- } \\
\text { Myers Squibb. }\end{array}$ \\
\hline
\end{tabular}

\section{Mathieu 2015}

Methods

24-week, multicenter, randomized, double-blind, placebocontrolled, parallel-group, phase 3 study in multiple centers across 7 countries 


\begin{tabular}{|c|c|}
\hline Participants & $\begin{array}{l}320 \text { initially randomized: placebo } n=160 \text {, dapagliflozin } 10 \mathrm{mg} n= \\
160 .\end{array}$ \\
\hline Interventions & $\begin{array}{l}\text { Randomized (blocked } 1: 1 \text { ratio within each stratum by HbA1c level) } \\
\text { to receive once-daily dapagliflozin } 10 \mathrm{mg} / \text { day plus saxagliptin and } \\
\text { metformin, or placebo. } \\
\text { Add on: saxagliptin } 5 \mathrm{mg} / \text { day and metformin. } \\
\text { Rescue mediation permitted in case of hyperglycemia. }\end{array}$ \\
\hline Outcomes & $\begin{array}{l}\text { Primary outcomes of the trial } \\
\text { mean change from baseline in HbA1c at week mean change from } \\
\text { baseline in HbA1c level after } 24 \text { weeks of double-blind treatment } \\
\text { with dapagliflozin versus placebo add-on to saxagliptin plus } \\
\text { metformin. } \\
\text { Secondary outcomes of the trial } \\
\text { mean change from baseline at } 24 \text { weeks in FPG level, 2-h } \\
\text { postprandial glucose (PPG) level following a liquid meal tolerance } \\
\text { test (MTT), } \\
\text { body weight, } \\
\text { mean proportion of patients achieving a therapeutic glycemic } \\
\text { response, defined as an HbA1c level of < } 7.0 \% \text { (53 mmol/mol), after } \\
24 \text { weeks, } \\
\text { proportion of patients rescued or discontinued from the study for } \\
\text { lack of efficacy, } \\
\text { change from baseline in the PPG area under the concentration- } \\
\text { time curve from } 0 \text { to } 180 \text { min (AUC0-180 min) during a liquid MTT, } \\
\text { change from baseline in serum lipid levels. } \\
\text { Adverse events and safety assessments } \\
\text { adverse events (AEs), } \\
\text { hypoglycemia, (major, requiring assistance and minor plasma } \\
\text { glucose concentration of <63 mg/dL, } \\
\text { vital signs, } \\
\text { severe cutaneous events, } \\
\text { decreased lymphocyte count, } \\
\text { decreased thrombocyte count, } \\
\text { opportunistic infection, } \\
\text { pancreatitis, } \\
\text { pancreatic cancer, } \\
\text { fracture, } \\
\text { severe hypersensitivity, }\end{array}$ \\
\hline
\end{tabular}




\begin{tabular}{|c|c|}
\hline & $\begin{array}{l}\text { worsening renal function, } \\
\text { genital infections, } \\
\text { urinary tract infections, } \\
\text { bladder neoplasm, } \\
\text { breast neoplasm, } \\
\text { volume depletion, } \\
\text { heart failure. } \\
\text { suspected cardiovascular AEs, } \\
\text { liver injury. }\end{array}$ \\
\hline Inclusion criteria & $\begin{array}{l}\text { adults with T2DM and inadequate glycemic control } \\
\mathrm{HbA} 1 \mathrm{c} \text { level of } 7.0-10.5 \%(53-91 \mathrm{mmol} / \mathrm{mol})\end{array}$ \\
\hline Exclusion criteria & $\begin{array}{l}\text { pregnancy, } \\
\text { cardiovascular events within } 3 \text { months of screening, } \\
\text { EGFR }<60 \mathrm{~mL} / \mathrm{min} / 1.73 \mathrm{~m}^{2} \\
\text { serum creatinine level of } \geq 1.5 \mathrm{mg} / \mathrm{dL} \text { in men or } \geq 1.4 \mathrm{mg} / \mathrm{dL} \text { in } \\
\text { women, } \\
\text { microscopic hematuria with no known cause in men, significant } \\
\text { hepatic disease. } \\
\text { on any antidiabetes medication, other than metformin and DPP-4 } \\
\text { inhibitors, for }>14 \text { days during the } 12 \text { weeks before screening. } \\
\text { uncontrolled hypertension (systolic blood pressure } \geq 160 \mathrm{~mm} \mathrm{Hg} \\
\text { and/or diastolic blood pressure } \geq 100 \mathrm{~mm} \mathrm{Hg} \text { ) were allowed to } \\
\text { enter the open-label period provided that their antihypertensive } \\
\text { therapy was adjusted appropriately. }\end{array}$ \\
\hline Notes & $\begin{array}{l}\text { ClinicalTrials.gov identifier: NCT01646320 } \\
\text { The results of a long-term extension will be presented in a } \\
\text { subsequent report }\end{array}$ \\
\hline
\end{tabular}

\section{Risk of bias table}

\begin{tabular}{||l|l|l||}
\hline Bias & $\begin{array}{l}\text { Authors' } \\
\text { judgement }\end{array}$ & Support for judgement \\
\hline $\begin{array}{l}\text { Random sequence } \\
\text { generation (selection } \\
\text { bias) }\end{array}$ & Low risk & $\begin{array}{l}\text { Randomly assigned by an interactive voice response } \\
\text { system in a centrally blocked 1:1 ratio within each } \\
\text { stratum (by HbA1c level) }\end{array}$ \\
\hline $\begin{array}{l}\text { Allocation } \\
\text { concealment (selection } \\
\text { bias) }\end{array}$ & Unclear risk & $\begin{array}{l}\text { Not described in detail but implied in computer } \\
\text { generated random system. }\end{array}$ \\
\hline
\end{tabular}




\begin{tabular}{|c|c|c|}
\hline $\begin{array}{l}\text { Blinding of participants } \\
\text { and personnel } \\
\text { (performance bias) }\end{array}$ & Low risk & Double blind. \\
\hline $\begin{array}{l}\text { Blinding of outcome } \\
\text { assessment (detection } \\
\text { bias) }\end{array}$ & Low risk & Double blind. \\
\hline $\begin{array}{l}\text { Incomplete outcome } \\
\text { data (attrition bias) }\end{array}$ & Low risk & $\begin{array}{l}\text { 301/320 completed (full analysis set; FAS). A last } \\
\text { observation carried forward (LOCF) approach was } \\
\text { used with terms for treatment group, stratum, and } \\
\text { baseline value in the statistical model. }\end{array}$ \\
\hline $\begin{array}{l}\text { Selective reporting } \\
\text { (reporting bias) }\end{array}$ & Low risk & $\begin{array}{l}\text { All clinically relevant outcomes are defined and } \\
\text { reported. }\end{array}$ \\
\hline Other bias & Unclear risk & $\begin{array}{l}\text { This study was funded by Bristol-Myers Squibb and } \\
\text { AstraZeneca. Medical writing support was funded by } \\
\text { AstraZeneca. Some of the study authors are } \\
\text { employees of Bristol-Myers Squibb and AstraZeneca. }\end{array}$ \\
\hline
\end{tabular}

\section{Matthaei 2015}

\begin{tabular}{|c|c|}
\hline Methods & $\begin{array}{l}\text { 52-week, international, multicenter, randomized, double-blind, } \\
\text { parallel group, placebo-controlled, phase 3b study in } 46 \text { centers in } 6 \\
\text { countries North America (Canada) and Europe (Czech Republic, } \\
\text { Germany, Poland, Slovak Republic, and Spain) }\end{array}$ \\
\hline Participants & $\begin{array}{l}218 \text { initially randomized dapagliflozin } 10 \mathrm{mg} / \text { day } n=109 \text {; or placebo } \\
n=109\end{array}$ \\
\hline Interventions & $\begin{array}{l}\text { Randomized } 1: 1 \text { dapagliflozin } 10 \mathrm{mg} \text { once daily or matched placebo } \\
\text { (oral dose) } \\
\text { Rescue medication permitted. } \\
\text { Add on: metformin, sulfonylurea }\end{array}$ \\
\hline Outcomes & $\begin{array}{l}\text { Primary outcomes of the trial } \\
\text { change from baseline to week } 52 \text { in HbA1c levels at } 24 \text { weeks } \\
\text { Secondary outcomes of the trial } \\
\text { change from baseline to week } 52 \text { in fasting plasma glucose (FPG) } \\
\text { total body weight, }\end{array}$ \\
\hline
\end{tabular}




\begin{tabular}{|c|c|}
\hline & $\begin{array}{l}\text { proportion of patients achieving a therapeutic glycemic response } \\
\text { (defined as HbA1c }<7.0 \% \text { ( } 53 \mathrm{mmol} / \mathrm{mol}) \text { ) } \\
\text { change from baseline to week } 52 \text { in seated SBP } \\
\text { change from baseline to week } 24 \text { in fasting lipids (total cholesterol, } \\
\text { LDL cholesterol, HDL cholesterol, LDL/HDL cholesterol ratio, and } \\
\text { triglycerides) } \\
\text { C-peptide } \\
\text { proportion of patients who discontinued for lack of efficacy or } \\
\text { were rescued for failing to maintain FPG below prespecified rescue } \\
\text { criteria. } \\
\text { Adverse events and safety assessments } \\
\text { cardiovascular events, } \\
\text { laboratory } \\
\text { values, } \\
\text { electrocardiogram, } \\
\text { vital signs, } \\
\text { hypoglycemic events, } \\
\text { calculated creatinine } \\
\text { clearance, } \\
\text { estimated glomerular filtration } \\
\text { rate, and } \\
\text { physical examination } \\
\text { undesirable medical condition or the deterioration of a pre-existing } \\
\text { medical condition } \\
\text { urinary tract infection } \\
\text { genital infection } \\
\text { hypoglycemia }\end{array}$ \\
\hline Inclusion criteria & $\begin{array}{l}\text { men } \\
\text { women who were not of childbearing potential } \\
\text { at least } 18 \text { years of age } \\
\text { T2DM } \\
\text { receiving a stable-dose combination therapy of metformin } \geq 1,500 \\
\mathrm{mg} / \text { day } \\
\text { inadequate glycemic control (HbA } 1 \mathrm{c} \geq 7.0 \%(53 \mathrm{mmol} / \mathrm{mol}) \text { to } \leq \\
10.5 \% \text { ( } 91 \mathrm{mmol} / \mathrm{mol}) \text { at randomization). }\end{array}$ \\
\hline Exclusion criteria & $\begin{array}{l}\text { type } 1 \text { diabetes, } \\
\text { BMI } \geq 45.0 \mathrm{~kg} / \mathrm{m}^{2} \text {, } \\
\text { serum creatinine value of } \geq 1.5 \mathrm{mg} / \mathrm{dL} \text { ( } 133 \mathrm{mmol} / \mathrm{L} \text { ) for men or } \\
\geq 1.4 \mathrm{mg} / \mathrm{dL} \text { ( } 124 \mathrm{mmol} / \mathrm{L} \text { ) for women, } \\
\text { unstable or rapidly progressing renal disease, } \\
\text { cardiovascular events } \\
\text { congestive heart failure (New York Heart Association Class IV), }\end{array}$ \\
\hline
\end{tabular}




\begin{tabular}{|c|c|}
\hline & systolic BP (SBP) $\geq 160 \mathrm{mmHg}$, or diastolic BP (DBP) $\geq 100 \mathrm{mmHg}$ \\
\hline Notes & $\begin{array}{l}\text { ClinicalTrials.gov identifier: NCT01392677 } \\
\text { Matthaei } 2015 \text { is a 28-week extension of the original 24-week trial } \\
\text { (Matthaei 2015a) (i.e. } 52 \text { weeks in total). We have included the data } \\
\text { from the 28-week extension into our analysis. } \\
\text { Treatment groups were balanced with respect to demographics and } \\
\text { diabetes-related baseline characteristics, with a higher proportion of } \\
\text { women in the dapagliflozin treatment arm. }\end{array}$ \\
\hline
\end{tabular}

\section{Risk of bias table}

\begin{tabular}{|c|c|c|}
\hline Bias & \begin{tabular}{|l} 
Authors' \\
judgement
\end{tabular} & Support for judgement \\
\hline $\begin{array}{l}\text { Random sequence } \\
\text { generation } \\
\text { (selection bias) }\end{array}$ & Low risk & An interactive response system (web or voice based). \\
\hline $\begin{array}{l}\text { Allocation } \\
\text { concealment } \\
\text { (selection bias) }\end{array}$ & Unclear risk & Not described. Implied by centralized randomization. \\
\hline $\begin{array}{l}\text { Blinding of } \\
\text { participants and } \\
\text { personnel } \\
\text { (performance bias) }\end{array}$ & Low risk & $\begin{array}{l}\text { Placebo identical in size, color, smell, taste, packaging, } \\
\text { and labelling. }\end{array}$ \\
\hline $\begin{array}{l}\text { Blinding of } \\
\text { outcome } \\
\text { assessment } \\
\text { (detection bias) }\end{array}$ & Low risk & $\begin{array}{l}\text { Placebo identical in size, color, smell, taste, packaging, } \\
\text { and labelling. }\end{array}$ \\
\hline $\begin{array}{l}\text { Incomplete } \\
\text { outcome data } \\
\text { (attrition bias) }\end{array}$ & Unclear risk & $\begin{array}{l}\text { 202/219 completed. } 1 \text { participant died before } \\
\text { randomization. } 216 / 219 \text { entered the full analysis set, } \\
\text { which included all randomized patients who received at } \\
\text { least one dose of study medication during the } 24 \text {-week } \\
\text { double-blind treatment period with a non-missing } \\
\text { baseline value and one or more post-baseline value for at } \\
\text { least one efficacy variable analyzed at week } 24 \text {. The } \\
\text { intention-to-treat principle was preserved despite the } \\
\text { exclusion of patients who took no study medication. The } \\
\text { primary safety analyses included all data regardless of }\end{array}$ \\
\hline
\end{tabular}




\begin{tabular}{||l||l|l||}
\hline \hline & & $\begin{array}{l}\text { rescue. All drops and losses to follow accounted for } \\
\text { "Approximately 93\% of the patients in each treatment } \\
\text { arm completed the 24-week double-blind treatment } \\
\text { period" }\end{array}$ \\
\hline $\begin{array}{l}\text { Selective reporting } \\
\text { (reporting bias) }\end{array}$ & Low risk & All clinically relevant outcomes reported \\
\hline Other bias & Unclear risk & $\begin{array}{l}\text { This study was sponsored by Bristol-Myers Squibb and } \\
\text { AstraZeneca. Medical writing assistance was funded by } \\
\text { AstraZeneca }\end{array}$ \\
\hline
\end{tabular}

\section{Nauck 2011}

\begin{tabular}{|c|c|}
\hline Methods & $\begin{array}{l}\text { 52-week randomized, double- blind, parallel-group, active- } \\
\text { controlled, phase III, non-inferiority trial with a 156- week extension } \\
\text { period ongoing at } 95 \text { sites in } 10 \text { countries (Argentina, France, } \\
\text { Germany, U.K., Italy, Mexico, the Netherlands, South Africa, Spain, } \\
\text { Sweden. }\end{array}$ \\
\hline Participants & $\begin{array}{l}814 \text { initially randomized. Dapagliflozin plus metformin } n=406 \text {; } \\
\text { glipizide plus metformin } n=408\end{array}$ \\
\hline Interventions & $\begin{array}{l}\text { Randomized in a 1:1 ratio to receive double-blind treatment with } \\
\text { dapagliflozin or glipizide uptitrated to maximally tolerated doses (up } \\
\text { to } 10 \text { and } 20 \mathrm{mg} \text {, respectively). Rescue medication was permitted. } \\
\text { Daily oral dose for } 52 \text { weeks. } \\
\text { Add on: metformin }\end{array}$ \\
\hline Outcomes & $\begin{array}{l}\text { Assessed at week } 52 \text {. } \\
\text { Primary outcomes of the trial } \\
\text { absolute change in HbA1c from baseline } \\
\text { Secondary outcomes of the trial } \\
\text { proportion of patients reporting at least one episode of } \\
\text { hypoglycemia } \\
\text { proportion of patients achieving a TBW decrease } \geq 5 \% \text { from } \\
\text { baseline } \\
\text { change from baseline body weight in patients with a baseline BMI } \\
\geq 30 \mathrm{~kg} / \mathrm{m}^{2} \text { and in those with baseline } \mathrm{BMI} \geq 27 \mathrm{~kg} / \mathrm{m}^{2} \text {, } \\
\text { waist circumference, } \\
\text { change in } \mathrm{HbA} 1 \mathrm{c} \text { in patients with an } \mathrm{HbA} 1 \mathrm{c} \text { of } \geq 7 \% \text { at baseline }\end{array}$ \\
\hline
\end{tabular}




\begin{tabular}{|c|c|}
\hline & $\begin{array}{l}\text { FPG } \\
\text { proportions of patients with } \mathrm{HbA} 1 \mathrm{c}<7 \% \text { at week } 52 \text { in patients } \\
\text { with baseline } \mathrm{HbA} 1 \mathrm{c} \geq 7 \% \text { and proportions of patients with } \mathrm{HbA} 1 \mathrm{c} \\
\leq 6.5 \% \text { at week } 52 \\
\text { absolute changes from baseline to week } 52 \text { for seated systolic and } \\
\text { diastolic blood pressure, } \\
\text { percent changes from baseline to week } 52 \text { for total cholesterol, } \\
\text { LDL cholesterol, HDL cholesterol triglycerides, and free fatty acids. } \\
\text { Adverse events and safety assessments } \\
\text { hypoglycemic events, } \\
\text { laboratory tests, } \\
\text { calculated creatinine clearance, } \\
\text { urinary glucose } \\
\text { creatinine ratio, } \\
\text { electrocardiographic and } \\
\text { physical examinations, } \\
\text { vital signs } \\
\text { genital infections and urinary tract infections. }\end{array}$ \\
\hline Inclusion criteria & $\begin{array}{l}\text { men and women } \\
\text { aged }>18 \text { years } \\
\text { inadequately controlled type } 2 \text { diabetes ( } \mathrm{HbA} 1 \mathrm{c}>6.5 \text { and } \leq 10 \% \text { ), a } \\
\text { maximum of } 25 \% \text { of randomized patients had a baseline } \mathrm{HbA} 1 \mathrm{c} \\
<7 \% \text { while receiving metformin or metformin and one other OAD } \\
\text { administered up to half-maximal dose for at least } 8 \text { weeks before } \\
\text { enrolment. } \\
\text { fasting plasma glucose (FPG) } \leq 15 \mathrm{mmol} / \mathrm{L} \text { and C-peptide } \\
\text { concentration of } \geq 0.33 \mathrm{nmol} / \mathrm{L} \text {. }\end{array}$ \\
\hline Exclusion criteria & $\begin{array}{l}\text { Diabetes related: type } 1 \text { diabetes; diabetes insipidus; } \\
\text { corticosteroid-induced type } 2 \text { diabetes; a history of diabetic } \\
\text { ketoacidosis or hyperosmolar non-ketotic coma; poorly controlled } \\
\text { diabetes characterized by polyuria/polydipsia with }>10 \% \text { weight } \\
\text { loss; use of insulin within } 1 \text { year of enrolment, except in the case of } \\
\text { hospitalization or use in gestational diabetes. } \\
\text { General: body mass index (BMI) }>45.0 \mathrm{~kg} / \mathrm{m} 2 \text {, } \\
\text { calculated creatinine clearance }<60 \mathrm{~mL} / \mathrm{min} \text {, } \\
\text { urine albumin:creatinine ratio }>203.4 \mathrm{mg} / \mathrm{mmol} \text {, } \\
\text { aspartate aminotransferase and/or alanine aminotransferase } \\
\text { and/or creatine kinase } \geq 3 \times \text { upper limit of normal range, } \\
\text { serum total bilirubin }>34 \mu \mathrm{mol} / \mathrm{L} \text {, } \\
\text { hemoglobin (Hb) } \leq 11 \mathrm{~g} / \mathrm{dL} \text { for men and } \leq 10 \mathrm{~g} / \mathrm{dL} \text { for women, } \\
\text { abnormal thyroid stimulating hormone level, } \\
\text { systolic blood pressure } \geq 180 \mathrm{mmHg} \text { and/or diastolic blood pressure }\end{array}$ \\
\hline
\end{tabular}




\begin{tabular}{|c|c|}
\hline & $\begin{array}{l}\geq 110 \mathrm{mmHg} \text {, } \\
\text { cardiovascular event within } 6 \text { months of enrolment; congestive } \\
\text { heart failure; } \\
\text { congenital renal glycosuria, } \\
\text { significant renal, hepatic, respiratory, hematological, oncological, } \\
\text { endocrine, immunological (including hypersensitivity to study } \\
\text { medications), } \\
\text { alcohol and/or substance misuse disorders, } \\
\text { pregnancy and/or lactation, } \\
\text { use of systemic corticosteroids equivalent to }>10 \mathrm{mg} \text { of oral } \\
\text { prednisolone within } 30 \text { days of enrolment, a history of bariatric } \\
\text { surgery; and use of weight loss medication within } 30 \text { days of } \\
\text { enrolment. }\end{array}$ \\
\hline Notes & $\begin{array}{l}\text { Only data from the } 52 \text {-week double-blind treatment period are used } \\
\text { in this analysis. } \\
\text { ClinicalTrials.gov identifier: NCT00660907 }\end{array}$ \\
\hline
\end{tabular}

\section{Risk of bias table}

\begin{tabular}{|l|l|l||}
\hline Bias & $\begin{array}{l}\text { Authors' } \\
\text { judgement }\end{array}$ & Support for judgement \\
\hline $\begin{array}{l}\text { Random sequence } \\
\text { generation } \\
\text { (selection bias) }\end{array}$ & Low risk & $\begin{array}{l}\text { Patients were randomized sequentially at study level } \\
\text { according to a predefined computer-generated } \\
\text { randomization scheme provided by AstraZeneca. }\end{array}$ \\
\hline $\begin{array}{l}\text { Allocation } \\
\text { concealment } \\
\text { (selection bias) }\end{array}$ & Unclear risk & $\begin{array}{l}\text { Not stated, but implied as allocation of study treatments } \\
\text { Was performed via an Interactive Web Response System } \\
\text { in balanced block sizes of 4, to ensure approximate } \\
\text { balance among treatment groups. }\end{array}$ \\
\hline $\begin{array}{l}\text { Blinding of } \\
\text { participants and } \\
\text { personnel } \\
\text { (performance bias) }\end{array}$ & Low risk & $\begin{array}{l}\text { Blinding of patients and investigators to study treatment } \\
\text { was achieved using a double-dummy technique. }\end{array}$ \\
\hline $\begin{array}{l}\text { Blinding of } \\
\text { outcome } \\
\text { assessment } \\
\text { (detection bias) }\end{array}$ & $\begin{array}{l}\text { Low risk } \\
\text { bot }\end{array}$ & $\begin{array}{l}\text { Not described if all outcome assessments were blinded } \\
\text { (e.g. coding of adverse events), but likely they were as } \\
\text { study describes the use of MedDRA terms and study } \\
\text { described as double blinded. }\end{array}$ \\
\hline $\begin{array}{l}\text { Incomplete } \\
\text { outcome data }\end{array}$ & $\begin{array}{l}\text { 622/814 completed the initial 52-week trial. Two analysis } \\
\text { sets were defined: the safety analysis set, consisting of all }\end{array}$ \\
\hline
\end{tabular}




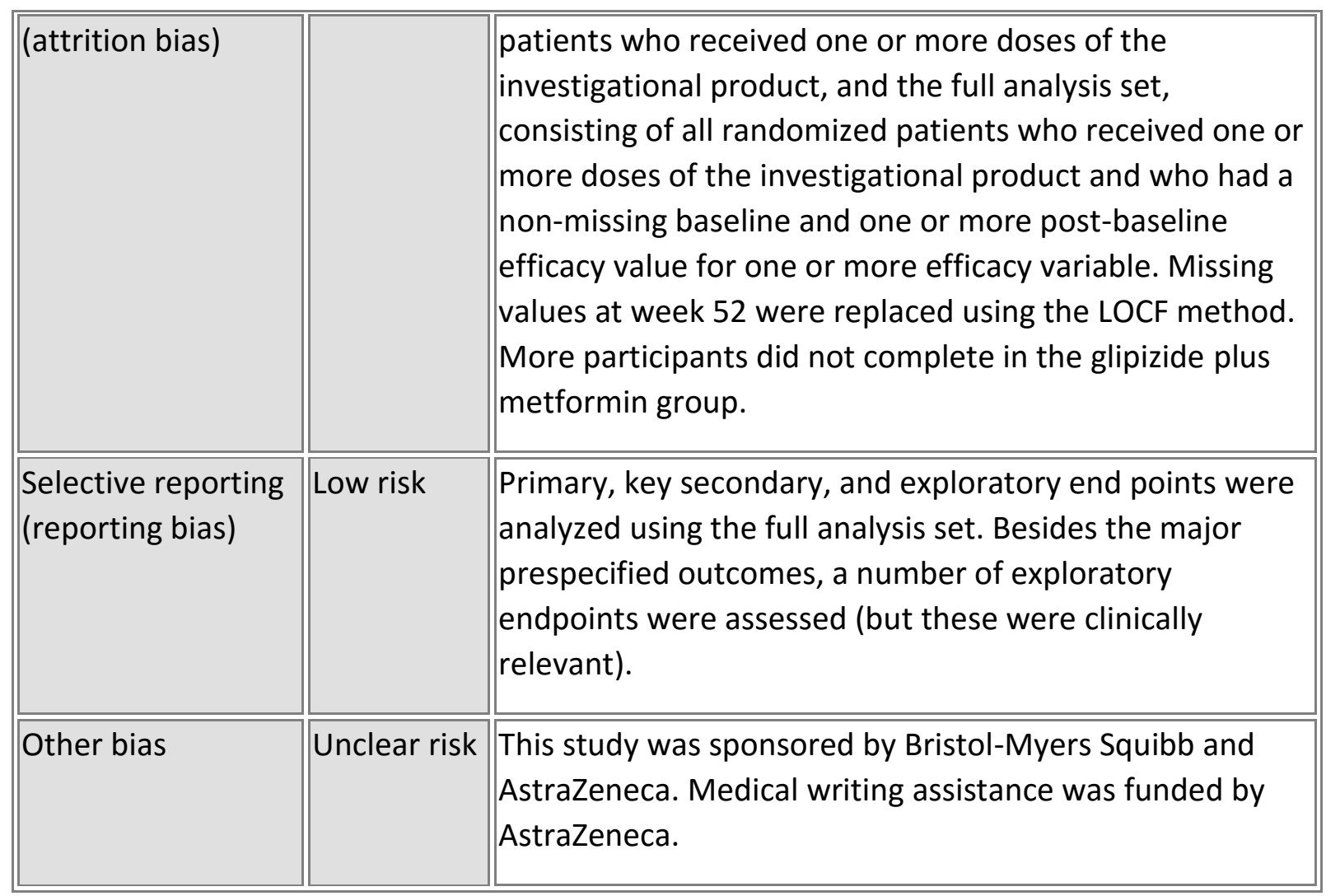

Ridderstråle 2013

\begin{tabular}{|c|c|}
\hline Methods & 104-week randomized, active-controlled, double-blind, phase 3 trial \\
\hline Participants & 1549 initially randomized, empagliflozin $n=769$; glimepiride $n=780$. \\
\hline Interventions & $\begin{array}{l}\text { 1:1 ratio empagliflozin ( } 25 \mathrm{mg} \text { once daily, orally) or glimepiride (1-4 } \\
\text { mg once daily, oral dose) for } 104 \text { weeks. } \\
\text { Add on: metformin immediate release and diet and exercise. } \\
\text { Rescue treatment permitted in case of hyperglycemia. }\end{array}$ \\
\hline Outcomes & $\begin{array}{l}\text { Primary outcomes of the trial } \\
\text { change from baseline in HbA1c concentrations at weeks } 52 \text { and } \\
104 . \\
\text { Secondary outcomes of the trial } \\
\text { changes from baseline in bodyweight, } \\
\text { systolic blood pressure, and diastolic blood pressure at weeks } 52 \\
\text { and } 104, \\
\text { percentage of patients who received rescue therapy (increases in } \\
\text { the dose of metformin or additional antidiabetes treatment) over } \\
104 \text { weeks, }\end{array}$ \\
\hline
\end{tabular}




\begin{tabular}{|c|c|}
\hline & $\begin{array}{l}\text { percentage of patients with HbA1c concentrations of at least } 7 \% \\
\text { who achieved a level of less than } 7 \% \text {, percentage of patients with } \\
\text { bodyweight reductions of more than } 5 \% \text {, and changes from } \\
\text { baseline in fasting plasma glucose at weeks } 52 \text { and } 104, \\
\text { change from baseline in HbA1c concentration at week } 104 \text { in } \\
\text { subgroup with baseline HbA1c of at least } 8.5 \% \text { and less than } 8.5 \% \text {, } \\
\text { lipids profiles cholesterol and triglycerides, } \\
\text { Substudies: sub-studies: changes from baseline in mean daily } \\
\text { glucose at weeks } 52 \text { and } 104 \text { were assessed with eight-point } \\
\text { glucose profiles; baseline in } 2 \text {-h post-prandial glucose at weeks } 52 \\
\text { and } 104 \text {; trunk fat, limb fat, total fat mass, fat-free mass, } \\
\text { abdominal visceral adipose tissue, and sub cutaneous adipose } \\
\text { tissue. } \\
\text { Adverse events and safety assessments } \\
\text { confirmed hypoglycemic events (plasma glucose } \leq 3.9 \text { mmol/L or } \\
\text { requiring assistance) up to weeks } 52 \text { and } 104, \\
\text { vital signs, } \\
\text { clinical laboratory findings, } \\
\text { urinary tract infection, genital infection, } \\
\text { volume depletion. }\end{array}$ \\
\hline Inclusion criteria & $\begin{array}{l}\text { Adults (aged } \geq 18 \text { years) } \\
\text { T2DM } \\
\text { BMI less than or equal to } 45 \mathrm{~kg} / \mathrm{m}^{2} \\
\text { HbA1c concentrations of } \geq 7 \text { to } 10 \% \text {, } \\
\text { receiving an unchanged dose of metformin immediate release } \\
\text { ( } \geq 1500 \mathrm{mg} / \text { day, maximum tolerated dose, or maximum dose } \\
\text { according to the local label) for at least } 12 \text { weeks before } \\
\text { randomization } \\
\text { If female: post-menopausal, or pre-menopausal and using } \\
\text { appropriate contraception; not pregnant/breastfeeding. }\end{array}$ \\
\hline Exclusion criteria & $\begin{array}{l}\text { eGFR of less than } 60 \mathrm{~mL} / \mathrm{min} \text { per } 1 \cdot 73 \mathrm{~m}^{2} \text { (Modified Diet Renal } \\
\text { Disease formula) } \\
\text { blood glucose concentration }>13 \cdot 3 \mathrm{mmol} / \mathrm{L} \text { after an overnight fast } \\
\text { during the placebo run-in, confirmed by a second measurement, } \\
\text { use of antidiabetes drugs other than metformin immediate release } \\
\text { any time during the } 12 \text { weeks before randomization. } \\
\text { bariatric surgery within } 2 \text { years; treatment with anti-obesity drugs } \\
\text { within } 3 \text { months of screening; any treatment leading to unstable } \\
\text { body weight } \\
\text { indication of liver disease (ALT, AST or alkaline phosphatase }>3 \mathrm{x} \\
\text { ULN) during screening or placebo run-in } \\
\text { cancer within } 5 \text { years (except basal cell carcinoma) }\end{array}$ \\
\hline
\end{tabular}




\begin{tabular}{||l||l||}
\hline \hline & $\begin{array}{l}\text { acute coronary syndrome, stroke or transient ischemic attack } \\
\text { within } 3 \text { months of informed consent } \\
\text { unstable red blood cells } \\
\text { treatment with systemic steroids } \\
\text { change in dose of thyroid hormones within } 6 \text { weeks of screening } \\
\text { any uncontrolled endocrine condition (except T2DM) } \\
\text { alcohol or drug abuse within 3 months of informed consent } \\
\text { taking an investigational drug within } 30 \text { days prior to receiving } \\
\text { study drug }\end{array}$ \\
\hline Notes & $\begin{array}{l}\text { ClinicalTrials.gov identifier: NCT01167881. } \\
\text { 52-week study, extended to } 104 \text { weeks. }\end{array}$ \\
\hline
\end{tabular}

Risk of bias table

\begin{tabular}{|c|c|c|}
\hline Bias & \begin{tabular}{|l} 
Authors' \\
judgement
\end{tabular} & Support for judgement \\
\hline $\begin{array}{l}\text { Random sequence } \\
\text { generation (selection } \\
\text { bias) }\end{array}$ & Low risk & $\begin{array}{l}\text { The study sponsor did the randomization, stratified by } \\
\mathrm{HbA} 1 \mathrm{c} \text { concentration at screening, eGFR, and region, } \\
\text { using an interactive response system with a } \\
\text { computer-generated } \\
\text { random sequence. }\end{array}$ \\
\hline $\begin{array}{l}\text { Allocation } \\
\text { concealment } \\
\text { (selection bias) }\end{array}$ & Unclear risk & $\begin{array}{l}\text { Not described, but implied in the computer-generated } \\
\text { stratified randomization sequence technique. }\end{array}$ \\
\hline $\begin{array}{l}\text { Blinding of participants } \\
\text { and personnel } \\
\text { (performance bias) }\end{array}$ & Low risk & $\begin{array}{l}\text { Patients and investigators were masked to treatment } \\
\text { assignment. Double-blind, double-dummy. }\end{array}$ \\
\hline $\begin{array}{l}\text { Blinding of outcome } \\
\text { assessment (detection } \\
\text { bias) }\end{array}$ & Low risk & $\begin{array}{l}\text { Patients and investigators were masked to treatment } \\
\text { assignment. Double-blind, double-dummy }\end{array}$ \\
\hline $\begin{array}{l}\text { Incomplete outcome } \\
\text { data (attrition bias) }\end{array}$ & Low risk & $\begin{array}{l}\text { 1300/1549 completed, } 1549 \text { included in full data } \\
\text { analysis set. All drops outs and losses to follow up } \\
\text { accounted for. }\end{array}$ \\
\hline $\begin{array}{l}\text { Selective reporting } \\
\text { (reporting bias) }\end{array}$ & Low risk & $\begin{array}{l}\text { All clinically relevant outcomes are defined and } \\
\text { reported. Lipid profile was specified in the study } \\
\text { protocol, but not reported in the main study report } \\
\text { (present in the appendix). }\end{array}$ \\
\hline
\end{tabular}




\begin{tabular}{|l|l|l||}
\hline Other bias & Unclear risk & $\begin{array}{l}\text { This study was sponsored by Boehringer Ingelheim } \\
\text { and Eli Lilly. Medical writing assistance, supported } \\
\text { financially by Boehringer Ingelheim. Some of the } \\
\text { trialists are employees of Boehringer Ingelheim. }\end{array}$ \\
\hline
\end{tabular}

Roden 2013

\begin{tabular}{|c|c|}
\hline Methods & $\begin{array}{l}24 \text { week, double-blind, parallel-group, randomized at } 124 \text { in } 9 \\
\text { countries }\end{array}$ \\
\hline Participants & $\begin{array}{l}899 \text { initially randomized: empagliflozin } 10 \mathrm{mg} n=224 \text {; empagliflozin } \\
25 \mathrm{mg} \mathrm{n}=224 \text {; sitagliptin } n=22\end{array}$ \\
\hline Interventions & $\begin{array}{l}\text { Randomly allocated patients (1:1:1:1) to empagliflozin } 10 \mathrm{mg}, 25 \mathrm{mg} \text {, } \\
\text { sitagliptin } 100 \mathrm{mg} \text { or placebo (oral dose) for } 24 \text { weeks. } \\
\text { Rescue medication permitted in case of hyperglycemia. } \\
\text { Add on: none }\end{array}$ \\
\hline Outcomes & $\begin{array}{l}\text { Primary outcomes of the trial } \\
\text { change from baseline in HbA1c at week } 24 \text {. } \\
\text { Secondary outcomes of the trial } \\
\text { change from baseline in bodyweight at week } 24 \text { and } \\
\text { change from baseline in systolic and diastolic blood pressures at } \\
\text { week } 24 \text {. } \\
\text { percentage of patients with HbA1c of at least } 7 \cdot 0 \% \text { at baseline who } \\
\text { had HbA1c lower than } 7 \cdot 0 \% \text { at week } 24 \text {, } \\
\text { change from baseline in fasting plasma glucose at week } 24 \text {, } \\
\text { percentage of patients with a greater than } 5 \cdot 0 \% \text { reduction in } \\
\text { bodyweight at week } 24, \\
\text { change from baseline in waist circumference at week } 24, \\
\text { percentage of patients who had uncontrolled blood pressure at } \\
\text { baseline who had controlled blood pressure (systolic blood } \\
\text { pressure }<130 \text { mm Hg and diastolic blood pressure }<80 \text { mm Hg) at } \\
\text { week } 24 . \\
\text { change from baseline in HbA1c at week } 24 \text { in subgroups of patients } \\
\text { with baseline HbA1c of at least } 8 \cdot 5 \% \text { and lower than } 8 \cdot 5 \% \text {. } \\
\text { use of rescue therapy } \\
\text { Adverse events and safety assessments } \\
\text { vital signs, } \\
\text { clinical laboratory parameters, }\end{array}$ \\
\hline
\end{tabular}




\begin{tabular}{|c|c|}
\hline & $\begin{array}{l}\text { confirmed hypoglycemic adverse events } \\
\text { urinary tract infection } \\
\text { genital infection }\end{array}$ \\
\hline Inclusion criteria & $\begin{array}{l}\text { men and women } \\
\text { with T2DM } \\
>18 \text { years ( } \geq 20 \text { years in Japan or } 18-65 \text { years in India) } \\
\text { BMI } \geq 45 \mathrm{~kg} / \mathrm{m}^{2} \text {, } \\
\text { insufficient glycemic control despite a diet and exercise regimen } \\
\mathrm{HbA} 1 \mathrm{c} 7 \cdot 0-10 \cdot 0 \% \text { (or } \mathrm{HbA} 1 \mathrm{c} 7 \cdot 0-9 \cdot 0 \% \text { in Germany) at screening. }\end{array}$ \\
\hline Exclusion criteria & $\begin{array}{l}\text { uncontrolled hyperglycemia (glucose concentration }>13.3 \mathrm{mmol} / \mathrm{L} \\
\text { after an overnight fast during the placebo run-in phase and } \\
\text { confirmed by a second measurement), } \\
\text { eGFR, estimated with the modification of diet in renal disease } \\
\text { (MDRD) equation) of }<50 \mathrm{~mL} / \mathrm{min} \text { per } 1.73 \mathrm{~m}^{2} \text { (or }<60 \mathrm{~mL} / \mathrm{min} \text { per } \\
1.73 \mathrm{~m}^{2} \text { in China), } \\
\text { contraindications to sitagliptin according to the local label, } \\
\text { treatment with antiobesity drugs within } 3 \text { months before informed } \\
\text { consent, } \\
\text { treatment with systemic steroids } \\
\text { change in dose of thyroid hormones within } 6 \text { weeks before } \\
\text { informed consent, } \\
\text { uncontrolled endocrine disorder apart from type } 2 \text { diabetes. }\end{array}$ \\
\hline Notes & $\begin{array}{l}\text { ClinicalTrials.gov identifier: NCT01177813. } \\
\text { 52-week non-randomized extension (NCT01289990) and open arm } \\
\text { with poor glycemic control (HbA1c >10\%), not included in meta- } \\
\text { analysis. }\end{array}$ \\
\hline
\end{tabular}

\section{Risk of bias table}

\begin{tabular}{|c|c|c|}
\hline Bias & $\begin{array}{l}\text { Authors' } \\
\text { judgement }\end{array}$ & Support for judgement \\
\hline $\begin{array}{l}\text { Random sequence } \\
\text { generation } \\
\text { (selection bias) }\end{array}$ & Low risk & $\begin{array}{l}\text { Patients with HbA1c } 7-10 \text { randomly allocated in a triple- } \\
\text { dummy manner (1:1:1:1 ratio) to oral in block sizes of four } \\
\text { and stratified by region (Asia, Europe, and North } \\
\text { America), HbA1c at screening } \geq 8.5 \% \text { ) and eGFR at } \\
\text { screening ( } \geq 90 \mathrm{~mL} / \mathrm{min} \text { per } 1.73 \mathrm{~m}^{2} \text {, } \\
60-89 \mathrm{~mL} / \mathrm{min} \text { per } 1.73 \mathrm{~m}^{2} \text {, and } 50-59 \mathrm{~mL} / \mathrm{min} \text { per } 1.73 \\
\mathrm{~m}^{2} \text { ). }\end{array}$ \\
\hline Allocation & Low risk & Interactive voice and Internet-based response system, \\
\hline
\end{tabular}




\begin{tabular}{|c|c|c|}
\hline $\begin{array}{l}\text { concealment } \\
\text { (selection bias) }\end{array}$ & & $\begin{array}{l}\text { with a computer generated random sequence, with no } \\
\text { access by the investigators. } \\
\text { "Access to the randomization code was strictly limited to } \\
\text { non-trial team functions including a randomization } \\
\text { operator, a trained person to generate the randomization } \\
\text { scheme, clinical trial supply unit staff responsible for } \\
\text { packaging and labelling, an independent randomization } \\
\text { statistician to verify and release the randomization } \\
\text { scheme, a system operator for clinical data systems to } \\
\text { perform the technical aspects of uploading the } \\
\text { randomization scheme, a dedicated contract research } \\
\text { organization (CRO) responsible for the interactive voice } \\
\text { and internet-based response system, and a dedicated } \\
\text { CRO supporting the Data Monitoring Committee. } \\
\text { Investigators did not have access to the } \\
\text { randomization code however, a code break was available } \\
\text { to the investigator" via the interactive voice and internet- } \\
\text { based response system in emergencies. }\end{array}$ \\
\hline $\begin{array}{l}\text { Blinding of } \\
\text { participants and } \\
\text { personnel } \\
\text { (performance bias) }\end{array}$ & Low risk & $\begin{array}{l}\text { Participants, investigators, and individuals involved in } \\
\text { analysis of trial data were masked to treatment } \\
\text { assignment during the randomized treatment period. } \\
\text { Medications administered in a triple-dummy manner. }\end{array}$ \\
\hline $\begin{array}{l}\text { Blinding of } \\
\text { outcome } \\
\text { assessment } \\
\text { (detection bias) }\end{array}$ & Low risk & $\begin{array}{l}\text { Investigators, and individuals involved in analysis of trial } \\
\text { data were masked to treatment assignment during the } \\
\text { randomized treatment period. }\end{array}$ \\
\hline $\begin{array}{l}\text { Incomplete } \\
\text { outcome data } \\
\text { (attrition bias) }\end{array}$ & Low risk & $\begin{array}{l}803 / 889 \text { completed. All } 880 \text { randomized were included in } \\
\text { the full analysis set. Drop outs and losses to follow up } \\
\text { accounted for. }\end{array}$ \\
\hline $\begin{array}{l}\text { Selective reporting } \\
\text { (reporting bias) }\end{array}$ & Low risk & All clinically relevant outcomes are defined and reported. \\
\hline Other bias & Unclear risk & $\begin{array}{l}\text { Boehringer Ingelheim as study sponsor was involved in } \\
\text { study design, data collection, and data analysis. Eli Lilly co- } \\
\text { sponsored the study but was not involved in study } \\
\text { design, data collection, or data analysis. Medical writing } \\
\text { assistance, supported financially by Boehringer Ingelheim. } \\
\text { Some of the trialists are employees of Boehringer } \\
\text { Ingelheim. }\end{array}$ \\
\hline
\end{tabular}


Rosenstock 2012

\begin{tabular}{|c|c|}
\hline Methods & $\begin{array}{l}12 \text { week randomized, double-blind, placebo-controlled, parallel- } \\
\text { group, multicenter, dose-ranging study in } 85 \text { study sites in } 12 \\
\text { countries. }\end{array}$ \\
\hline Participants & $\begin{array}{l}451 \text { initially randomized: canagliflozin } 50 \mathrm{mg} n=64 \text {; canagliflozin } \\
100 \mathrm{mg} n=64 \text {; canagliflozin } 300 \mathrm{mg} n=64 \text {; canagliflozin sitagliflozin } \\
n=65 ; \text { placebo } n=65 \text {. }\end{array}$ \\
\hline Interventions & $\begin{array}{l}\text { Seven treatment groups: canagliflozin at doses of } 50,100,200, \text { or } \\
300 \mathrm{mg} \text { once daily (QD) or } 300 \mathrm{mg} \text { twice daily (BID); sitagliptin } 100 \\
\text { mg QD, versus placebo for } 12 \text { weeks } \\
\text { Add on: metformin }\end{array}$ \\
\hline Outcomes & $\begin{array}{l}\text { Primary outcomes of the trial } \\
\text { change in A1C from baseline to week } 12 \text {. } \\
\text { Secondary outcomes of the trial } \\
\text { fasting plasma glucose (FPG), } \\
\text { overnight urinary glucose-to-creatinine } \\
\text { (UGlucose-to-UCreatinine) ratio, } \\
\text { body weight, } \\
\text { change in the percentage of participants with A1C } \geq 7.0 \% \text { and } \\
\geq 6.5 \% \\
\text { change in fasting serum lipids (triglycerides, HDL cholesterol, LDL } \\
\text { cholesterol, total cholesterol, and total cholesterol- to-HDL } \\
\text { cholesterol ratio), } \beta \text {-Cell function was indirectly assessed by } \\
\text { changes in homeostasis model assessment } 2 \text { (HOMA2) index of } \beta \text { - } \\
\text { cell function (HOMA2-\%B) } \\
\text { Adverse events and safety assessments } \\
\text { adverse event (AE) reports, } \\
\text { vital signs, } \\
12-\text { lead electrocardiograms, } \\
\text { physical examinations, } \\
\text { laboratory assessments. } \\
\text { vaginal swabs for Candida culture and } \\
\text { urine cultures were to be obtained from all at baseline and week } \\
12 \text { and at the time of a vulvovaginal AE } \\
\text { hypoglycemia. }\end{array}$ \\
\hline Inclusion criteria & men and women \\
\hline
\end{tabular}




\begin{tabular}{||l||l||}
\hline \hline & aged 18-65 years (mean 52.9 years, SD 8.1)) \\
& T2DM for at least 3 months (mean duration 6.0 SD 4.9) \\
& A1C level $\geq 7 \%$ and $\geq 10.5 \%$, \\
& on metformin monotherapy at a stable ( $>3$ months) dose of $>1,500$ \\
& $\mathrm{~m} /$ day, \\
& stable body weight and BMI $25-45 \mathrm{~kg} / \mathrm{m}^{2}\left(24-45 \mathrm{~kg} / \mathrm{m}^{2}\right.$ for those of \\
& $\begin{array}{l}\text { Asian descent), } \\
\text { serum creatinine levels }<1.5 \mathrm{mg} / \mathrm{dL} \text { for men and }<1.4 \mathrm{mg} / \mathrm{dL} \text { for } \\
\text { women. }\end{array}$ \\
\hline Exclusion criteria & None described. \\
\hline Notes & ClinicalTrials.gov identifier: NCT00642278 \\
\hline
\end{tabular}

\section{Risk of bias table}

\begin{tabular}{|c|c|c|}
\hline Bias & \begin{tabular}{|l|} 
Authors' \\
judgement
\end{tabular} & Support for judgement \\
\hline $\begin{array}{l}\text { Random sequence } \\
\text { generation (selection } \\
\text { bias) }\end{array}$ & Unclear risk & Not described. \\
\hline $\begin{array}{l}\text { Allocation } \\
\text { concealment } \\
\text { (selection bias) }\end{array}$ & Unclear risk & Not described. \\
\hline $\begin{array}{l}\text { Blinding of } \\
\text { participants and } \\
\text { personnel } \\
\text { (performance bias) }\end{array}$ & Low risk & Double blind. \\
\hline $\begin{array}{l}\text { Blinding of outcome } \\
\text { assessment } \\
\text { (detection bias) }\end{array}$ & Low risk & Double blind. \\
\hline $\begin{array}{l}\text { Incomplete outcome } \\
\text { data (attrition bias) }\end{array}$ & Low risk & $\begin{array}{l}402 / 451 \text { initially randomized completed the } 12 \text { week } \\
\text { intervention. } \\
\text { Efficacy analyses were based on the intent-to-treat } \\
\text { analysis set (all randomized participants), and safety } \\
\text { analyses included all intent-to-treat participants who } \\
\text { received at least one dose of the study medication. } \\
\text { Discontinuation because of AEs was documented ( } 11 \\
\text { overall, } 9 \text { of whom were in the canagliflozin groups) and }\end{array}$ \\
\hline
\end{tabular}




\begin{tabular}{||l||l|l||}
\hline \hline & & $\begin{array}{l}\text { the numbers of drops out and losses to follow up and } \\
\text { the reasons for this were described. }\end{array}$ \\
\hline $\begin{array}{l}\text { Selective reporting } \\
\text { (reporting bias) }\end{array}$ & Low risk & $\begin{array}{l}\text { All major clinically relevant outcomes are reported. } \\
\text { The reporting of urogenital infections is divided into } \\
\text { many different terms (i.e. VVAEs in female participants, } \\
\text { Urinary tract AEs, Symptomatic genital infections, } \\
\text { Urinary tract infection, vulvovaginal mycotic infection). } \\
\text { it is unclear how these items overlap. }\end{array}$ \\
\hline Other bias & Unclear risk & $\begin{array}{l}\text { This study was funded by Janssen Global Services, LLC. } \\
\text { Editorial assistance was supported by Janssen Global } \\
\text { Services, LLC. }\end{array}$ \\
\hline \hline
\end{tabular}

\section{Rosenstock 2012a}

\begin{tabular}{|c|c|}
\hline Methods & $\begin{array}{l}\text { 24-week, randomized, double-blind, placebo-controlled, parallel } \\
\text { group, } 105 \text { sites in Argentina, Canada, India, Mexico, Peru, } \\
\text { Philippines, Taiwan, and United States }\end{array}$ \\
\hline Participants & $\begin{array}{l}420 \text { initially randomized dapagliflozin } 5 \mathrm{mg}(n=141) \text { or } 10 \mathrm{mg}(\mathrm{n}= \\
140) \text { or placebo }(n=139)\end{array}$ \\
\hline Interventions & $\begin{array}{l}\text { Randomized patients received double-blind dapagliflozin } 5 \text { or } 10 \mathrm{mg} \\
\text { or placebo every day (oral administration) with open-label } \\
\text { pioglitazone } 30 \text { or } 45 \mathrm{mg} / \text { day, stratified by pre-enrollment diabetes } \\
\text { treatment } \\
\text { group A and B. } \\
\text { Add on: pioglitazone } \\
\text { Rescue medication permitted }\end{array}$ \\
\hline Outcomes & $\begin{array}{l}\text { Primary outcomes of the trial } \\
\text { change at } 24 \text { weeks from baseline in } \mathrm{HbA} 1 \mathrm{c} \\
\text { Secondary outcomes of the trial } \\
\text { change from baseline in FPG, } \\
\text { postprandial glucose (PPG) measured by } 120 \text {-min post-challenge } \\
\text { response to an oral glucose tolerance test } \\
\text { total body weight. } \\
\text { Adverse events and safety assessments }\end{array}$ \\
\hline
\end{tabular}




\begin{tabular}{|c|c|}
\hline & $\begin{array}{l}\text { adverse events, } \\
\text { laboratory abnormalities, } \\
\text { vital signs } \\
\text { hypoglycemia } \\
\text { urinary tract infection (UTI) and } \\
\text { genital infection } \\
\text { seated blood pressure }\end{array}$ \\
\hline Inclusion criteria & $\begin{array}{l}\text { men and women } \\
\text { T2DM } \\
\geq 18 \text { years } \\
\text { fasting C-peptide } \geq 1.0 \mathrm{ng} / \mathrm{mL} \\
\text { BMI } \leq 45.0 \mathrm{~kg} / \mathrm{m} 2 \text { entered group A or } \mathrm{B} \text {. } \\
\text { Group A patients had received } \geq 12 \text { weeks of pioglitazone } 30 \text { or } 45 \\
\mathrm{mg} / \text { day and had } \mathrm{HbA} 1 \mathrm{c} \geq 7.0 \text { and } \leq 10.5 \% \text {. } \\
\text { Group B patients were drug naive for the previous } 10 \text { weeks with } \\
\mathrm{HbA} 1 \mathrm{c} \geq 8.0 \text { and } \leq 11.0 \% \text { or had received pioglitazone } 15 \mathrm{mg} / \text { day or } \\
\text { any dose of rosiglitazone with } \mathrm{HbA} 1 \mathrm{c} \geq 8.0 \text { and } \leq 11.0 \% \text { or had } \\
\text { received } \geq 8 \text { weeks of metformin } 1700 \mathrm{mg} / \text { day or sulfonylurea less } \\
\text { than or equal to half the maximal dose with HbA1c } \geq 7.0 \text { and } \\
\geq 11.0 \% \text {. } \\
\text { Group B patients could not be on }>1 \text { oral antidiabetic medication, } \\
\text { and patients on more than half the maximum dose of sulfonylurea } \\
\text { or metformin were excluded. }\end{array}$ \\
\hline Exclusion criteria & $\begin{array}{l}\text { aspartate or alanine aminotransferases }>2.5 \text { times the upper limit } \\
\text { of normal, } \\
\text { total bilirubin }>2.0 \mathrm{mg} / \mathrm{dL} \text {, } \\
\text { serum creatinine } \geq 2.0 \mathrm{mg} / \mathrm{dL} \text {, } \\
\text { urine albumin/creatinine ratio }>1,800 \mathrm{mg} / \mathrm{g} \text {, } \\
\text { calculated creatinine } \\
\text { clearance }<50 \mathrm{~mL} / \mathrm{min} \text {, } \\
\text { congestive heart failure class III or IV }\end{array}$ \\
\hline Notes & $\begin{array}{l}\text { Study MB102030 } \\
\text { Background treatment pioglitazone, open label rescue medication } \\
\text { (metformin or SU) from week 24-48. Data from these patients } \\
\text { excluded from efficacy data. } 48 \text { weeks safety data are included in } \\
\text { our analyses. }\end{array}$ \\
\hline
\end{tabular}

Risk of bias table

\begin{tabular}{|l|l|l||}
\hline Bias & Authors' & Support for judgement \\
\hline
\end{tabular}




\begin{tabular}{|c|c|c|}
\hline & judgement & \\
\hline $\begin{array}{l}\text { Random sequence } \\
\text { generation (selection } \\
\text { bias) }\end{array}$ & Unclear risk & Not described in detail. Stated to be randomized \\
\hline $\begin{array}{l}\text { Allocation concealment } \\
\text { (selection bias) }\end{array}$ & Unclear risk & Not described. \\
\hline $\begin{array}{l}\text { Blinding of participants } \\
\text { and personnel } \\
\text { (performance bias) }\end{array}$ & Low risk & Double blind placebo. \\
\hline $\begin{array}{l}\text { Blinding of outcome } \\
\text { assessment (detection } \\
\text { bias) }\end{array}$ & Low risk & Double blind placebo. \\
\hline $\begin{array}{l}\text { Incomplete outcome } \\
\text { data (attrition bias) }\end{array}$ & Low risk & $\begin{array}{l}367 / 420 \text { completed. All dropouts and losses to } \\
\text { follow-up accounted for. }\end{array}$ \\
\hline $\begin{array}{l}\text { Selective reporting } \\
\text { (reporting bias) }\end{array}$ & Unclear risk & $\begin{array}{l}\text { All clinically relevant outcomes are defined and } \\
\text { reported }\end{array}$ \\
\hline Other bias & Unclear risk & $\begin{array}{l}\text { Study supported by Bristol-Myers Squibb and } \\
\text { AstraZeneca. Some of the trialists are Employees of } \\
\text { Bristol Myers squib. Bristol-Myers Squibb provided } \\
\text { editorial assistants }\end{array}$ \\
\hline
\end{tabular}

\section{Rosenstock 2013}

\begin{tabular}{||l|l|}
\hline Methods & $\begin{array}{l}\text { 12-week randomized dose-ranging, double-blind, placebo-controlled } \\
\text { trial at } 104 \text { centers in } 16 \text { countries }\end{array}$ \\
\hline Participants & $\begin{array}{l}495 \text { initially randomized: placebo } n=71,1 \mathrm{mg} \text { empagliflozin } \mathrm{n}=71, \\
5 \mathrm{mg} \text { empagliflozin } \mathrm{n}=71,10 \mathrm{mg} \text { empagliflozin }=71,25 \mathrm{mg} \\
\text { empagliflozin } \mathrm{n}=70,50 \mathrm{mg} \text { empagliflozin } \mathrm{n}=70, \text { open-label sitagliptin } \\
\mathrm{n}=70) .\end{array}$ \\
\hline Interventions & $\begin{array}{l}\text { Randomized to receive one of five doses of empagliflozin }(1,5,10, \\
25 \text { or } 50 \mathrm{mg} \text { oral dose, once daily), or placebo, } \\
\text { Add on: metformin }\end{array}$ \\
\hline \hline Outcomes & $\begin{array}{l}\text { Primary outcomes of the trial } \\
\text { change in HbA1c from baseline to week } 12 \text { with empagliflozin }\end{array}$ \\
\hline
\end{tabular}




\begin{tabular}{|c|c|}
\hline & 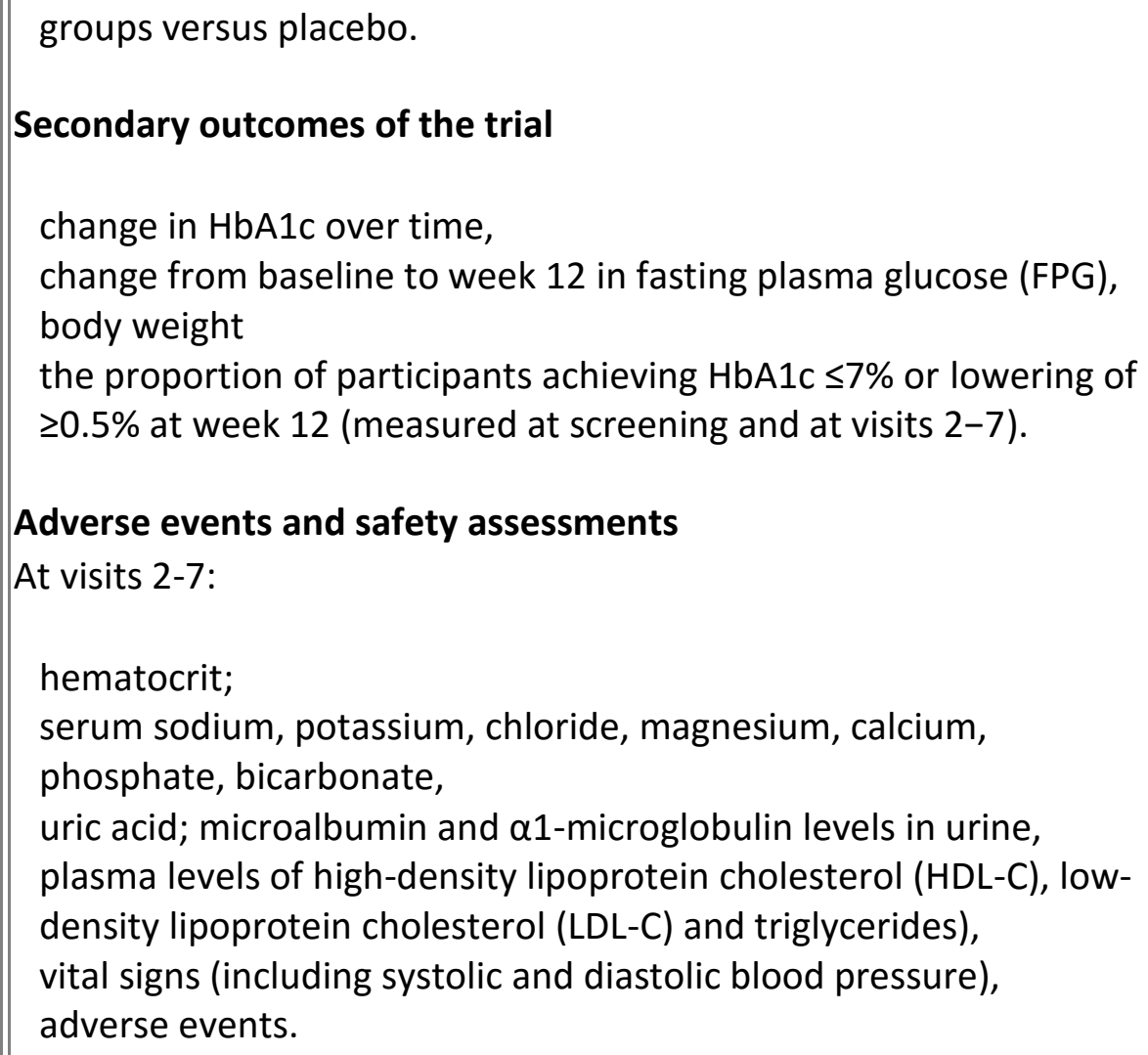 \\
\hline Inclusion criteria & $\begin{array}{l}\text { men and women } \\
\text { aged } \geq 18 \text { to }<80 \text { years } \\
\text { T2DM } \\
\text { body mass index (BMI) } \leq 40 \mathrm{~kg} / \mathrm{m} 2 \text {; } \\
\text { previous treatment with metformin alone or with metformin and } \\
\text { one other oral antidiabetic agent (OAD) } \\
\text { unchanged antidiabetic therapy for } \geq 10 \text { weeks prior to screening } \\
\text { including stable metformin therapy ( } \geq 1500 \mathrm{mg} / \text { day or maximum } \\
\text { tolerated dose); } \\
\text { hemoglobin } \mathrm{A} 1 \mathrm{c}(\mathrm{HbA} 1 \mathrm{c} \text { ) of } \geq 6.5 \text { to } \leq 9 \% \text { for patients on metformin } \\
\text { and one other } \mathrm{OAD} \text {, discontinued at the start of the washout } \\
\text { period; } \\
\mathrm{HbA} 1 \mathrm{c}>7 \text { to } \leq 10 \% \text { for those on metformin monotherapy; and } \\
\mathrm{HbA} 1 \mathrm{c}>7 \text { to } 10 \% \text { for all participants at start of placebo run-in } \\
\text { period. }\end{array}$ \\
\hline Exclusion criteria & $\begin{array}{l}\text { history of myocardial infarction, stroke or transient ischemic attack } \\
\text { within } 6 \text { months } \\
\text { impaired hepatic or renal function; } \\
\text { diseases of the central nervous system; } \\
\text { chronic or clinically relevant acute infections; } \\
\text { history of clinically relevant allergy/hypersensitivity; } \\
\text { treatment with thiazolidinediones, } \\
\text { glucagon-like peptide-1 (GLP-1) analogues or insulin within } 3\end{array}$ \\
\hline
\end{tabular}


months.

Notes

ClinicalTrials.gov identifier: NCT00749190

Dosage $25 \mathrm{mg}$ versus placebo, as add-on to metformin, included in our analyses. Open label Sitagliptin arm not included.

\section{Risk of bias table}

\begin{tabular}{|c|c|c|}
\hline Bias & $\begin{array}{l}\text { Authors' } \\
\text { judgement }\end{array}$ & Support for judgement \\
\hline $\begin{array}{l}\text { Random sequence } \\
\text { generation (selection } \\
\text { bias) }\end{array}$ & Low risk & $\begin{array}{l}\text { Random allocation, by an interactive voice } \\
\text { response system. }\end{array}$ \\
\hline $\begin{array}{l}\text { Allocation concealment } \\
\text { (selection bias) }\end{array}$ & Unclear risk & $\begin{array}{l}\text { Not fully described, but implied in the } \\
\text { randomization schedule generated by interactive } \\
\text { voice response system. }\end{array}$ \\
\hline $\begin{array}{l}\text { Blinding of participants } \\
\text { and personnel } \\
\text { (performance bias) }\end{array}$ & Low risk & Double blind placebo. \\
\hline $\begin{array}{l}\text { Blinding of outcome } \\
\text { assessment (detection } \\
\text { bias) }\end{array}$ & Low risk & Double blind placebo \\
\hline $\begin{array}{l}\text { Incomplete outcome data } \\
\text { (attrition bias) }\end{array}$ & Low risk & $\begin{array}{l}\text { 473/495 completed. Full analysis set based on a } \\
\text { modified last observation carried forward. Drop } \\
\text { outs and losses to follow up accounted for. }\end{array}$ \\
\hline $\begin{array}{l}\text { Selective reporting } \\
\text { (reporting bias) }\end{array}$ & Low risk & $\begin{array}{l}\text { All clinically relevant outcomes are defined and } \\
\text { reported. }\end{array}$ \\
\hline Other bias & Unclear risk & $\begin{array}{l}\text { This study was funded by Boehringer Ingelheim. } \\
\text { Medical writing assistance, supported financially } \\
\text { by Boehringer Ingelheim. }\end{array}$ \\
\hline
\end{tabular}

\section{Rosenstock 2014}

Methods 52-week, randomized, double-blind, placebo-controlled, parallelgroup study in 104 centers across 14 countries. 


\begin{tabular}{|c|c|}
\hline Participants & $\begin{array}{l}563 \text { initially randomized: placebo } n=188 \text {, empagliflozin } 10 \mathrm{mg} \mathrm{n}= \\
186 \text {, or empagliflozin } 25 \mathrm{mg} \mathrm{n}=189 .\end{array}$ \\
\hline Interventions & $\begin{array}{l}\text { Randomized (1:1:1) to receive once-daily empagliflozin } 10 \mathrm{mg} \text {, } \\
\text { empagliflozin } 25 \mathrm{mg} \text {, or placebo. } \\
\text { Add on: MDI insulin, with or without metformin, for } 52 \text { weeks (oral } \\
\text { dose). Diet and exercise counselling based on local } \\
\text { recommendations. Rescue medication permitted in case of } \\
\text { hyperglycemia. }\end{array}$ \\
\hline Outcomes & $\begin{array}{l}\text { Primary outcomes of the trial } \\
\text { change from baseline in } \mathrm{HbA} 1 \mathrm{c} \text { at week } 18 \text {. } \\
\text { Secondary outcomes of the trial } \\
\text { changes from baseline at week } 52 \text { in insulin daily dose, body } \\
\text { weight, and HbA1c. } \\
\text { body weight at week } 18 \text {; } \\
\text { changes from baseline at weeks } 18 \text { and } 52 \text { in fasting plasma } \\
\text { glucose (FPG), } \\
\text { systolic blood pressure (SBP), and diastolic blood pressure (DBP); } \\
\text { percentage of patients with HbA1c } \geq 7 \% \text { ( } \geq 53 \text { mmol/mol) at } \\
\text { baseline who had HbA1c }<7 \% \text { at weeks } 18 \text { and } 52 ; \\
\text { use of rescue therapy. } \\
\text { Adverse events and safety assessments } \\
\text { vital signs, } \\
\text { clinical laboratory parameters, } \\
\text { adverse events (AEs) up to } 7 \text { days after the last dose of study drug } \\
\text { confirmed hypoglycemic AEs } \\
\text { with urinary tract } \\
\text { genital tract infections. }\end{array}$ \\
\hline Inclusion criteria & $\begin{array}{l}\text { obese adults ( } \mathrm{BMI} \geq 30 \text { and } \leq 45 \mathrm{~kg} / \mathrm{m} 2 \text { ) } \\
\text { T2DM } \\
\text { insufficient glycemic control ( } \mathrm{HbA} 1 \mathrm{c} \geq 7.5 \text { to } \leq 10 \% \text { ( } \$ 58 \text { to } \# 86 \\
\mathrm{mmol} / \mathrm{mol} \text { ) at screening) despite diet and exercise counselling and } \\
\text { treatment with } \mathrm{MDI} \text { insulin (total daily dose }>60 \text { international } \\
\text { units) alone or in combination with metformin } \\
\text { (immediate or extended release, } \geq 1,500 \mathrm{mg} / \text { day, maximum } \\
\text { tolerated dose, or maximum dose according to the local label). } \\
\text { For } \geq 12 \text { weeks prior to randomization, insulin dose was not to be } \\
\text { changed by }>10 \% \text { and metformin dose was to be unchanged. }\end{array}$ \\
\hline
\end{tabular}




\begin{tabular}{|c|c|}
\hline & Premixed insulins were not permitted. \\
\hline Exclusion criteria & $\begin{array}{l}\text { uncontrolled hyperglycemia (glucose level }>13.3 \mathrm{mmol} / \mathrm{L} \text { after an } \\
\text { overnight fast; } \\
\text { acute coronary syndrome, stroke, or transient ischemic attack } \\
\text { within } 3 \text { months prior to consent; } \\
\text { indication of liver disease; } \\
\text { impaired renal function during screening or run-in (eGFR using the } \\
\text { modification of diet and renal disease equation }<60 \mathrm{~mL} / \mathrm{min} / 1.73 \\
\mathrm{~m}^{2} \text { ); } \\
\text { gastrointestinal surgeries that induce malabsorption; } \\
\text { cancer (except for basal cell carcinoma); } \\
\text { disorders causing hemolysis or unstable erythrocytes; } \\
\text { treatment with systemic steroids; } \\
\text { change in dosage of thyroid hormones within } 6 \text { weeks; } \\
\text { treatment with anti-obesity drugs; } \\
\text { alcohol or drug abuse; } \\
\text { investigational drug intake within } 30 \text { days of intake of study drug. }\end{array}$ \\
\hline Notes & ClinicalTrials.gov identifier: NCT01306214 \\
\hline
\end{tabular}

\section{Risk of bias table}

\begin{tabular}{||l||l|l||}
\hline Bias & $\begin{array}{l}\text { Authors' } \\
\text { judgement }\end{array}$ & Support for judgement \\
\hline $\begin{array}{l}\text { Random sequence } \\
\text { generation } \\
\text { (selection bias) }\end{array}$ & Low risk & $\begin{array}{l}\text { Randomization was undertaken using a third-party } \\
\text { interactive voice- and web-response system and was } \\
\text { stratified by HbA1c, eGFR, region (Europe, North America, } \\
\text { Latin America), and background antidiabetes therapy } \\
\text { (insulin alone, insulin plus metformin). }\end{array}$ \\
\hline $\begin{array}{l}\text { Allocation } \\
\text { concealment } \\
\text { (selection bias) }\end{array}$ & $\begin{array}{l}\text { Unclear risk } \\
\text { Not fully described, but implied in the third-party } \\
\text { interactive voice- and web-response system. }\end{array}$ \\
\hline $\begin{array}{l}\text { Blinding of } \\
\text { participants and } \\
\text { personnel } \\
\text { (performance bias) }\end{array}$ & Low risk & Double-blind. \\
\hline $\begin{array}{l}\text { Blinding of } \\
\text { outcome } \\
\text { assessment }\end{array}$ & Low risk & Double-blind. \\
\hline
\end{tabular}




\begin{tabular}{|c|c|c|}
\hline (detection bias) & & \\
\hline $\begin{array}{l}\text { Incomplete } \\
\text { outcome data } \\
\text { (attrition bias) }\end{array}$ & Low risk & $\begin{array}{l}475 / 563 \text { Completed the } 52 \text {-week intervention period. The } \\
\text { full analysis dataset (FAS) uses a LOCF method and is } \\
\text { patients treated with study medication who had a } \\
\text { baseline HbA1c measurement. The PPS-completers- } 52 \text { set } \\
\text { is patients in the FAS who were on treatment up to day } \\
357 \text { and did not have important protocol violations. The } \\
\text { reasons for drops outs and losses to follow up are in the } \\
\text { supplementary publication of this study. }\end{array}$ \\
\hline $\begin{array}{l}\text { Selective reporting } \\
\text { (reporting bias) }\end{array}$ & Low risk & All clinically relevant outcomes are defined and reported. \\
\hline Other bias & Unclear risk & $\begin{array}{l}\text { This study was funded by Boehringer Ingelheim and Eli } \\
\text { Lilly. Some of the trialists are employees of Boehringer } \\
\text { Ingelheim. }\end{array}$ \\
\hline
\end{tabular}

\section{Rosenstock 2015}

\begin{tabular}{|c|c|}
\hline Methods & $\begin{array}{l}\text { 78-week, randomized, double-blind, placebo-controlled, parallel- } \\
\text { group study in } 97 \text { centers across } 7 \text { countries }\end{array}$ \\
\hline Participants & $\begin{array}{l}494 \text { initially randomized: placebo empagliflozin } 10 \mathrm{mg}(\mathrm{n}=169) \\
\text { empagliflozin } 25 \mathrm{mg}(\mathrm{n}=155) \text { or placebo }(n=170)\end{array}$ \\
\hline Interventions & $\begin{array}{l}\text { Randomized (1:1:1) to receive once-daily empagliflozin } 10 \mathrm{mg} \text {, } \\
\text { empagliflozin } 25 \mathrm{mg} \text {, or placebo. } \\
\text { Add on: insulin with or without metformin and sulfonyl ureas } \\
\text { Rescue mediation permitted in case of hyperglycemia. }\end{array}$ \\
\hline Outcomes & $\begin{array}{l}\text { Primary outcomes of the trial } \\
\text { change from baseline in } \mathrm{HbA} 1 \mathrm{c} \text { at week } 18 \text {. } \\
\text { Secondary outcomes of the trial } \\
\text { changes from baseline to week } 78 \text { in basal insulin dose and HbA1c. } \\
\text { Changes from baseline to weeks } 18 \text { and } 78 \text { : } \\
\text { FPG } \\
\text { bodyweight, } \\
\text { percentage with } \mathrm{HbA} 1 \mathrm{c} \geq 7 \%(\geq 53 \mathrm{mmol} / \mathrm{mol}) \text { at baseline who had }\end{array}$ \\
\hline
\end{tabular}




\begin{tabular}{|c|c|}
\hline & $\begin{array}{l}\text { HbA1c }<7 \%(<53 \mathrm{mmol} / \mathrm{mol}) \text { at weeks } 18 \text { and } 78 \text {. } \\
\text { systolic (SBP) } \\
\text { diastolic blood pressure (DBP). } \\
\text { Adverse events and safety assessments } \\
\text { vital signs, } \\
\text { clinical laboratory measures } \\
\text { lipid variables } \\
\text { adverse events including included all events with an onset after the } \\
\text { first dose and up to } 7 \text { days after the last dose of study medication, } \\
\text { confirmed hypoglycemic AEs (plasma glucose } \leq 3.9 m m o l / l \\
\text { ( } \leq 70 m g / d l) \text { and/or requiring assistance), } \\
\text { suspected and confirmed urinary tract infections (i.e. confirmed } \\
\text { infections were those that led to hospitalization or } \\
\text { discontinuations of the study drug), } \\
\text { genital infections. }\end{array}$ \\
\hline Inclusion criteria & $\begin{array}{l}\text { adults with T2DM } \\
\text { BMI } \leq 45 \mathrm{~kg} / \mathrm{m}^{2} \text {, } \\
\text { inadequately controlled type } 2 \text { diabetes ( } \mathrm{HbA} 1 \mathrm{c}>7 \text { to } \leq 10 \% \text { ( }>53 \text { to } \\
\leq 86 \mathrm{mmol} / \mathrm{mol} \text { ) at screening), despite treatment with basal glargine } \\
\text { or detemir insulin ( } \geq 20 \mathrm{IU} / \text { day) or } \mathrm{NPH} \text { insulin ( } \geq 14 \mathrm{IU} / \mathrm{day} \text {; at a } \\
\text { dose unchanged by }>10 \% \text { of baseline value for } \geq 12 \text { weeks before } \\
\text { randomization), with or without metformin and/or sulphonylurea } \\
\text { use (unchanged for } \geq 12 \text { weeks prior to randomization). }\end{array}$ \\
\hline Exclusion criteria & $\begin{array}{l}\text { uncontrolled hyperglycemia [glucose level }>13.3 \mathrm{mmol} / \mathrm{l} \\
\text { (>240mg/dl) after an overnight fast or }>22.2 \mathrm{mmol} / \mathrm{l}(>400 \mathrm{mg} / \mathrm{dl} \text { ) } \\
\text { from a random assessment during placebo run-in), } \\
\text { frequent hypoglycemic events on basal insulin therapy (in the } \\
\text { opinion of the investigator), } \\
\text { myocardial infarction, } \\
\text { stroke or transient ischemic attack }<3 \text { months before consent, } \\
\text { eGFR }<30 \mathrm{ml} / \mathrm{min} / 1.73 \mathrm{~m}^{2} \text {, } \\
\text { bariatric surgery, } \\
\text { investigational drug intake within } 2 \text { months of consent, } \\
\text { treatment with anti-obesity drugs, } \\
\text { any oral anti diabetes medication (other than metformin or } \\
\text { sulphonylurea), } \\
\text { chronic short-acting insulin or glucagon-like peptide- } 1 \text { receptor } \\
\text { agonists within } 3 \text { months of consent, } \\
\text { impaired hepatic function (serum alanine aminotransferase, } \\
\text { aspartate aminotransferase, or alkaline phosphatase }>3 \text { times the } \\
\text { upper limit of normal), } \\
\text { history of cancer (except for basal cell and squamous cell skin }\end{array}$ \\
\hline
\end{tabular}




\begin{tabular}{||l||l||}
\hline \hline & $\begin{array}{l}\text { cancer) within } 5 \text { years, } \\
\text { contraindication to background anti-diabetes medication according } \\
\text { to the local label, } \\
\text { disorders causing hemolysis or unstable red blood cells; treatment } \\
\text { with systemic steroids at time of consent, } \\
\text { change in dosage of thyroid hormones within } 6 \text { weeks prior to } \\
\text { consent, } \\
\text { alcohol or drug abuse. }\end{array}$ \\
\hline Notes & ClinicalTrials.gov identifier: NCT01011868 \\
\hline
\end{tabular}

\section{Risk of bias table}

\begin{tabular}{|c|c|c|}
\hline Bias & \begin{tabular}{|l} 
Authors' \\
judgement
\end{tabular} & Support for judgement \\
\hline $\begin{array}{l}\text { Random sequence } \\
\text { generation (selection } \\
\text { bias) }\end{array}$ & Low risk & $\begin{array}{l}\text { Randomization was performed using a third-party } \\
\text { interactive voice and web response system, and was } \\
\text { stratified by HbA1c at screening ( }<8.5 \%(<69 \mathrm{mmol} / \mathrm{mol} \\
\geq 8.5 \%(\geq 69 \mathrm{mmol} / \mathrm{mol}) \text { ) and center. }\end{array}$ \\
\hline $\begin{array}{l}\text { Allocation } \\
\text { concealment } \\
\text { (selection bias) }\end{array}$ & Unclear risk & $\begin{array}{l}\text { Not fully described, but implied in the third-party } \\
\text { interactive voice- and web-response system. }\end{array}$ \\
\hline $\begin{array}{l}\text { Blinding of } \\
\text { participants and } \\
\text { personnel } \\
\text { (performance bias) }\end{array}$ & Low risk & $\begin{array}{l}\text { Stated to be double blind, placebo controlled although } \\
\text { no details given. }\end{array}$ \\
\hline $\begin{array}{l}\text { Blinding of outcome } \\
\text { assessment (detection } \\
\text { bias) }\end{array}$ & Low risk & $\begin{array}{l}\text { Stated to be double blind, placebo controlled although } \\
\text { no details given. }\end{array}$ \\
\hline $\begin{array}{l}\text { Incomplete outcome } \\
\text { data (attrition bias) }\end{array}$ & Low risk & $\begin{array}{l}429 / 494 \text { completed } 18 \text { weeks treatment, } 360 / 494 \\
\text { completed } 78 \text { weeks. The full analysis set comprised } \\
\text { randomized patients treated with } \geq 1 \text { dose of study } \\
\text { drug and who had a baseline HbA1c value) at week } 18 \text {. }\end{array}$ \\
\hline $\begin{array}{l}\text { Selective reporting } \\
\text { (reporting bias) }\end{array}$ & Low risk & $\begin{array}{l}\text { All clinically relevant outcomes are defined and } \\
\text { reported. }\end{array}$ \\
\hline Other bias & Unclear risk & $\begin{array}{l}\text { This study was funded by Boehringer Ingelheim and Eli } \\
\text { Lilly. Some of the trialists are employees of Boehringer } \\
\text { Ingelheim. }\end{array}$ \\
\hline
\end{tabular}




\begin{tabular}{|c|c|}
\hline Methods & $\begin{array}{l}\text { 24-week, multicenter, randomized, double-blind, active-controlled, } \\
\text { parallel- group phase } 3 \text { study in eight countries (Canada, Mexico, } \\
\text { Poland, Puerto Rico, Republic of Korea, Romania, South Africa, } \\
\text { United States) }\end{array}$ \\
\hline Participants & $\begin{array}{l}534 \text { initially randomized: } 179 \text { saxagliptin + dapagliflozin + metformin; } \\
176 \text { saxagliptin + metformin; } 179 \text { dapagliflozin + metformin }\end{array}$ \\
\hline Interventions & $\begin{array}{l}\text { Randomized 1:1:1 using a centralized blocked randomization } \\
\text { schedule to receive saxagliptin ( } 5 \mathrm{mg} / \text { day) and dapagliflozin (10 } \\
\mathrm{mg} / \text { day) plus MET (SAXA+Dapagliflozin 10mg+MET), saxagliptin (5 } \\
\mathrm{mg} / \text { day) and placebo plus MET } \\
\text { (SAXA+MET), or dapagliflozin ( } 10 \mathrm{mg} / \text { day) and placebo plusMET } \\
\text { (Dapagliflozin } 10 \mathrm{mg}+\mathrm{MET} \text { ) for } 24 \text { weeks. Other anti-diabetic } \\
\text { medications prohibited during the screening and treatment periods. } \\
\text { Add on: metformin } \\
\text { Rescue medication open-label rescue medication, including insulin } \\
\text { or other antidiabetic medications, except metformin, GLP-1 receptor } \\
\text { agonists, and other DPP-4 inhibitors or SGLT2 inhibitors, was given. }\end{array}$ \\
\hline Outcomes & $\begin{array}{l}\text { Primary outcomes of the trial } \\
\text { adjusted mean change from baseline in } \mathrm{HbA} 1 \mathrm{c} \text { after } 24 \text { weeks of } \\
\text { double-blind treatment. } \\
\text { Secondary outcomes of the trial } \\
\text { adjusted mean change from baseline at } 24 \text { weeks in } 2-\mathrm{h} \\
\text { postprandial glucose (PPG), } \\
\text { adjusted mean change from baseline at } 24 \text { weeks in FPG, } \\
\text { adjusted mean proportion of patients achieving a therapeutic } \\
\text { glycemic response, defined as HbA1c }>7.0 \% \text { ( } 53 \text { mmol/mol), after } \\
24 \text { weeks, } \\
\text { adjusted mean change from baseline in body weight. } \\
\text { PPG after the administration of a liquid meal } \\
\text { adjusted mean changes from baseline at } 24 \text { weeks in fasting serum } \\
\text { lipids. } \\
\text { Adverse events and safety assessments } \\
\text { adverse events (AEs), } \\
\text { hypoglycemia, } \\
\text { laboratory abnormalities, }\end{array}$ \\
\hline
\end{tabular}




\begin{tabular}{|c|c|}
\hline & $\begin{array}{l}\text { vital signs. } \\
\text { severe cutaneous events, } \\
\text { decreased lymphocyte count, } \\
\text { decreased thrombocyte count, } \\
\text { opportunistic infection, } \\
\text { pancreatitis, } \\
\text { hepatic AEs, } \\
\text { fracture, } \\
\text { hypersensitivity, } \\
\text { worsening renal function, } \\
\text { genital infections, } \\
\text { urinary tract infections, } \\
\text { bladder neoplasm, } \\
\text { breast neoplasm } \\
\text { blood pressure (investigators adjusted antihypertensive therapy as } \\
\text { needed). }\end{array}$ \\
\hline Inclusion criteria & $\begin{array}{l}\geq 18 \text { years, mean age of } 54 \\
\text { T2DM } \\
\text { inadequate glycemic control, defined as } \mathrm{HbA} 1 \mathrm{c} \geq 8.0 \% \text { and } \leq 12.0 \% \\
\text { ( } 64-108 \mathrm{mmol} / \mathrm{mol} \text { ) } \\
\text { on stable metformin therapy ( } \geq 1,500 \mathrm{mg} / \mathrm{day} \text { ) for } \geq 8 \text { weeks } \\
\text { C peptide concentrations } \geq 1.0 \mathrm{ng} / \mathrm{mL} \\
\mathrm{BMI} \leq 45.0 \mathrm{~kg} / \mathrm{m}^{2} \text { at screening. }\end{array}$ \\
\hline Exclusion criteria & $\begin{array}{l}\text { pregnancy } \\
\text { uncontrolled hypertension (systolic blood pressure } \geq 160 \mathrm{mmHg} \text { and } \\
\text { diastolic blood pressure } \geq 100 \mathrm{mmHg} \text { ) at randomization, } \\
\text { fasting plasma glucose (FPG) } \geq 270 \mathrm{mg} / \mathrm{dL} \text { during the 4-week lead-in } \\
\text { period, } \\
\text { cardiovascular disease within } 3 \text { months of screening, } \\
\text { congestive heart failure (New York Heart Association functional } \\
\text { class IV), } \\
\text { eGFR } 60 \mathrm{~mL} / \mathrm{min} / 1.73 \mathrm{~m} 2 \text { or } \\
\text { serum creatinine } \geq 1.5 \mathrm{mg} / \mathrm{dL} \text { in men or } \geq 1.4 \mathrm{mg} / \mathrm{dL} \text { in women, } \\
\text { significant hepatic disease } \\
\text { any antidiabetic medication, other than metformin, for more than } \\
14 \text { days during the } 12 \text { weeks before screening. }\end{array}$ \\
\hline \multirow[t]{2}{*}{ Notes } & ClinicalTrials.gov identifier: NCT01606007 \\
\hline & $\begin{array}{l}\text { Patient demographics and baseline characteristics were balanced } \\
\text { across treatment groups }\end{array}$ \\
\hline
\end{tabular}

Risk of bias table 


\begin{tabular}{|c|c|c|}
\hline Bias & \begin{tabular}{|l} 
Authors' \\
judgement
\end{tabular} & Support for judgement \\
\hline $\begin{array}{l}\text { Random sequence } \\
\text { generation (selection } \\
\text { bias) }\end{array}$ & Low risk & $\begin{array}{l}\text { Randomized 1:1:1 using a centralized blocked } \\
\text { randomization schedule }\end{array}$ \\
\hline $\begin{array}{l}\text { Allocation } \\
\text { concealment } \\
\text { (selection bias) }\end{array}$ & Unclear risk & $\begin{array}{l}\text { Not described but implied by computerized } \\
\text { randomization. }\end{array}$ \\
\hline $\begin{array}{l}\text { Blinding of } \\
\text { participants and } \\
\text { personnel } \\
\text { (performance bias) }\end{array}$ & Low risk & Double blind placebo. \\
\hline $\begin{array}{l}\text { Blinding of outcome } \\
\text { assessment } \\
\text { (detection bias) }\end{array}$ & Low risk & Double blind placebo. \\
\hline $\begin{array}{l}\text { Incomplete outcome } \\
\text { data (attrition bias) }\end{array}$ & Low risk & $\begin{array}{l}\text { 534/590 completed. All dropouts and lost to follow-up } \\
\text { accounted for. The primary efficacy data set included } \\
\text { all randomized patients who received at least one dose } \\
\text { of a study medication during the double-blind } \\
\text { treatment period. }\end{array}$ \\
\hline $\begin{array}{l}\text { Selective reporting } \\
\text { (reporting bias) }\end{array}$ & Low risk & All clinical relevant outcomes are defined and reported. \\
\hline Other bias & Unclear risk & $\begin{array}{l}\text { This study was funded by Bristol-Myers Squibb and } \\
\text { AstraZeneca. Medical writing support funded from } \\
\text { Bristol Myers squib and AstraZeneca. }\end{array}$ \\
\hline
\end{tabular}

\section{Ross 2015}

\begin{tabular}{|c|c|}
\hline Methods & $\begin{array}{l}\text { 16-week, randomized, double-blind, placebo-controlled, parallel- } \\
\text { group study in multiple centers across Europe, North America, Latin } \\
\text { America) }\end{array}$ \\
\hline Participants & $\begin{array}{l}983 \text { initially randomized: empagliflozin } 12.5 \mathrm{mg} \text { twice daily }(n=219), \\
25 \mathrm{mg} \text { once daily ( } n=218), 5 \mathrm{mg} \text { twice daily }(n=219) \text { or } 10 \mathrm{mg} \text { once } \\
\text { daily }(n=220) \text {, or placebo }(n=107) \text {. }\end{array}$ \\
\hline Interventions & $\begin{array}{l}\text { Randomized } 2: 2: 2: 2: 1 \text { to receive empagliflozin } 12.5 \mathrm{mg} \text { twice } \\
\text { daily, } 25 \mathrm{mg} \text { once daily, } 5 \mathrm{mg} \text { twice daily or } 10 \mathrm{mg} \text { once daily, or }\end{array}$ \\
\hline
\end{tabular}




\begin{tabular}{|c|c|}
\hline & $\begin{array}{l}\text { placebo, for } 16 \text { weeks } \\
\text { Add on: diet and exercise counselling based on local } \\
\text { recommendations. } \\
\text { Rescue mediation permitted in case of hyperglycemia } \\
(>13.3 \mathrm{mmol} / \mathrm{l}) \text {. }\end{array}$ \\
\hline Outcomes & $\begin{array}{l}\text { Primary outcomes of the trial } \\
\text { change from baseline in HbA1c at week } 16 \text {. } \\
\text { Secondary outcomes of the trial } \\
\text { change from baseline in fasting plasma glucose (FPG) at week 16, } \\
\text { proportion of patients with HbA1c } \geq 7 \% \text { at baseline who had HbA1c } \\
<7 \% \text { at week 16, } \\
\text { changes from baseline at week 16, } \\
\text { weight, } \\
\text { systolic blood pressure (SBP), } \\
\text { diastolic blood pressure (DBP). } \\
\text { Adverse events and safety assessments } \\
\text { confirmed hypoglycemic AEs (defined as AEs with plasma glucose } \\
\leq 3.9 m m o l / l \text { and/or requiring assistance). } \\
\text { urinary tract infection (UTI), } \\
\text { genital infection, } \\
\text { increased urination, } \\
\text { volume depletion. }\end{array}$ \\
\hline Inclusion criteria & $\begin{array}{l}\text { adults with T2DM } \\
\text { BMI } \leq 45 \mathrm{~kg} / \mathrm{m}^{2} \text {, } \\
\text { glycated hemoglobin (HbA1c) level of } \geq 7 \text { and } \leq 10 \% \text { at screening } \\
\text { (despite a diet and exercise regimen), } \\
\text { treatment with a stable dose of metformin } I R \geq 1500 \mathrm{mg} / \text { day) for } \\
\geq 12 \text { weeks before randomization. }\end{array}$ \\
\hline Exclusion criteria & $\begin{array}{l}\text { uncontrolled hyperglycemia (plasma glucose }>13.3 \mathrm{mmol} / \mathrm{l} \text { after an } \\
\text { overnight fast during a } 2 \text {-week placebo run-in period, confirmed by } \\
\text { a second measurement), } \\
\text { renal impairment (estimated creatinine clearance rate }<60 \mathrm{ml} / \mathrm{min} \\
\text { using the Cockcroft-Gault formula), } \\
\text { indication of liver disease (serum alanine aminotransferase, } \\
\text { aspartate aminotransferase, or alkaline phosphatase }>3 \times \text { upper } \\
\text { limit of normal) at screening or during the placebo run-in, } \\
\text { acute coronary syndrome, }\end{array}$ \\
\hline
\end{tabular}




\begin{tabular}{||l||l||}
\hline \hline & $\begin{array}{l}\text { stroke, } \\
\text { transient ischemic attack within } 3 \text { months prior to consent, } \\
\text { had received anti-obesity drugs within } 3 \text { months of consent, had } \\
\text { undergone bariatric surgery within } 2 \text { years, } \\
\text { any uncontrolled endocrine disorder except T2DM, } \\
\text { had received any antidiabetes agent other than metformin IR in } \\
\text { the } 12 \text { weeks prior to consent. }\end{array}$ \\
\hline Notes & $\begin{array}{l}\text { EudraCT number 2012-000905-53 } \\
\text { NB: we compared the 25mg arm with placebo }\end{array}$ \\
\hline
\end{tabular}

\section{Risk of bias table}

\begin{tabular}{|c|c|c|}
\hline Bias & \begin{tabular}{|l} 
Authors' \\
judgement
\end{tabular} & Support for judgement \\
\hline $\begin{array}{l}\text { Random sequence } \\
\text { generation (selection } \\
\text { bias) }\end{array}$ & Low risk & $\begin{array}{l}\text { Implied by the declaration that 'randomization was } \\
\text { stratified by region (Europe, North America, Latin } \\
\text { America) and by HbA1c }(<8.5 \text { and } \geq 8.5 \%) \text { and estimatec } \\
\text { glomerular filtration rate }(\leq 60-89 \mathrm{ml} / \mathrm{min} / 1.73 \mathrm{~m} 2 \text { and } \\
\geq 90 \mathrm{ml} / \mathrm{min} / 1.73 \mathrm{~m} 2 \text { ) at screening'. }\end{array}$ \\
\hline $\begin{array}{l}\text { Allocation } \\
\text { concealment } \\
\text { (selection bias) }\end{array}$ & Unclear risk & No details given about allocation concealment. \\
\hline $\begin{array}{l}\text { Blinding of } \\
\text { participants and } \\
\text { personnel } \\
\text { (performance bias) }\end{array}$ & Low risk & Stated to be double blind, but no details given. \\
\hline $\begin{array}{l}\text { Blinding of outcome } \\
\text { assessment } \\
\text { (detection bias) }\end{array}$ & Low risk & Stated to be double blind, but no details given. \\
\hline $\begin{array}{l}\text { Incomplete outcome } \\
\text { data (attrition bias) }\end{array}$ & Low risk & $\begin{array}{l}\text { 916/983 completed 16-week treatment period. Al drop } \\
\text { outs and losses to follow up accounted for. The full } \\
\text { analysis dataset uses a LOCF method and is patients } \\
\text { treated with study medication } \geq 1 \text { dose of study } \\
\text { drug and had a baseline and on-treatment HbA1c } \\
\text { value). }\end{array}$ \\
\hline $\begin{array}{l}\text { Selective reporting } \\
\text { (reporting bias) }\end{array}$ & Low risk & $\begin{array}{l}\text { All clinically relevant outcomes are defined and } \\
\text { reported. }\end{array}$ \\
\hline
\end{tabular}




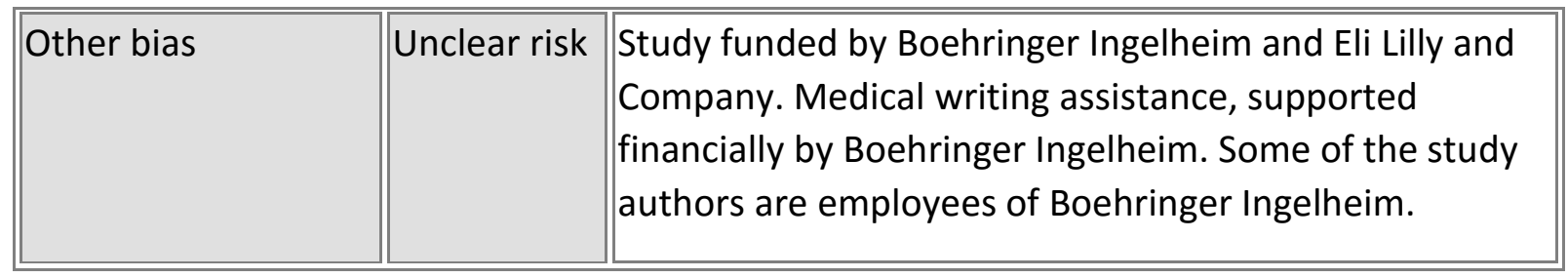

\section{Schernthaner 2013}

\begin{tabular}{|c|c|}
\hline Methods & $\begin{array}{l}52 \text { week, randomized, double-blind, active- controlled, multicenter } \\
\text { study phase } 3 \text { study at } 140 \text { centers in } 17 \text { countries }\end{array}$ \\
\hline Participants & $\begin{array}{l}755 \text { initially randomized: canagliflozin } 300 \mathrm{mg} n=378 \text {; sitagliflozin } n= \\
378 \text { ) }\end{array}$ \\
\hline Interventions & $\begin{array}{l}\text { Canagliflozin } 300 \mathrm{mg} \text { versus sitagliptin } 100 \mathrm{mg} \text { once daily (1:1) (oral } \\
\text { dose) for } 52 \text { weeks. } \\
\text { Add on: metformin }\end{array}$ \\
\hline Outcomes & $\begin{array}{l}\text { Primary outcomes of the trial } \\
\text { Change in A1C from baseline to week } 52 . \\
\text { Secondary outcomes of the trial } \\
\text { change from baseline in FPG, } \\
\text { systolic blood pressure (BP), } \\
\text { percent change from baseline in body weight, } \\
\text { triglycerides, and HDL cholesterol (HDL-C). } \\
\text { proportion of subjects reaching A1C }>7.0 \% \text { (53 mmol/mol) and } \\
\text { >6.5\% (48 mmol/mol), } \\
\text { change in diastolic BP, } \\
\text { percent change in other fasting plasma lipids. } \\
\text { Indices of } \beta \text {-cell function ( } \beta C F \text { ) (HOMA2-\%B), } \\
\text { proinsulin/insulin ratio, and proinsulin/ C-peptide ratio, } \\
\text { a subset underwent a FS-MMTT and postprandial glucose } \\
\text { measurements. } \\
\text { Adverse events and safety assessments } \\
\text { adverse events (AEs), } \\
\text { clinical laboratory tests, } \\
\text { vital sign measurements, } \\
\text { physical examinations, } \\
\text { self-monitored blood glucose, } \\
12 \text {-lead electrocardiograms, } \\
\text { hypoglycemic episodes, }\end{array}$ \\
\hline
\end{tabular}




\begin{tabular}{|c|c|}
\hline & $\begin{array}{l}\text { genital mycotic infections and urinary tract infections (UTIs), } \\
\text { incidence of hypoglycemia. }\end{array}$ \\
\hline Inclusion criteria & $\begin{array}{l}\text { men and women } 18 \text { years of age or older } \\
\text { T2DM } \\
\text { using stable metformin and sulfonylurea therapy. } \\
\text { (metformin } \geq 2,000 \mathrm{mg} / \text { day (or } \geq 1,500 \mathrm{mg} / \text { day if unable to tolerate } \\
\text { a higher dose); sulfonylurea at half-maximal labelled dose or } \\
\text { more), } \\
\text { A1C } \geq 7.0 \% \text { ( } 53 \mathrm{mmol} / \mathrm{mol} \text { ) and } \leq 10.5 \%(91 \mathrm{mmol} / \mathrm{mol} \text { ), } \\
\text { met all other enrolment criteria }\end{array}$ \\
\hline Exclusion criteria & $\begin{array}{l}\text { (FPG) or fasting self-monitored blood glucose measurements } \geq 16.7 \\
\mathrm{mmol} / \mathrm{L} \text { ( } 300 \mathrm{mg} / \mathrm{dL} \text { ), or both, during the pre-treatment phase; } \\
\text { history of type } 1 \text { diabetes, } \\
\text { cardiovascular disease, } \\
\text { uncontrolled hypertension, } \\
\text { treatment with either a PPARy agonist, ongoing insulin therapy, } \\
\text { another SGLT2 inhibitor, or any other AHA (other than metformin } \\
\text { and a sulfonylurea) within } 12 \text { weeks before screening, } \\
\text { eGFR } 55 \mathrm{~mL} / \mathrm{min} / 1.73 \mathrm{~m}^{2} \text { ( } 60 \mathrm{~mL} / \mathrm{min} / 1.73 \mathrm{~m}^{2} \text { if based on } \\
\text { restriction of metformin use in the metformin local label), } \\
\text { serum creatinine } \geq 124 \mathrm{mmol} / \mathrm{L} \text { (men) and } \geq 115 \mathrm{mmol} / \mathrm{L} \text { (women). }\end{array}$ \\
\hline \multirow[t]{2}{*}{ Notes } & ClinicalTrials.gov identifier: NCT01137812 \\
\hline & $\begin{array}{l}\text { The study consisted of a 52-week double-blind treatment phase, and } \\
\text { a 4-week follow-up period data from which are included in our } \\
\text { analyses. }\end{array}$ \\
\hline
\end{tabular}

\section{Risk of bias table}

\begin{tabular}{||l||l|l||}
\hline Bias & $\begin{array}{l}\text { Authors' } \\
\text { judgement }\end{array}$ & Support for judgement \\
\hline $\begin{array}{l}\text { Random sequence } \\
\text { generation } \\
\text { (selection bias) }\end{array}$ & Low risk & $\begin{array}{l}\text { Interactive Voice Response System/ Interactive Web } \\
\text { Response System. The computer-generated } \\
\text { randomization schedule was prepared by the sponsor } \\
\text { before the study, and randomization was balanced using } \\
\text { permuted blocks with the following two stratification } \\
\text { criteria: whether the pre randomization A1C was } \geq 9.0 \% \\
\text { (75 mmol/mol) and whether a subject underwent the } \\
\text { frequently sampled mixed-meal tolerance test (FS- } \\
\text { MMTT). }\end{array}$ \\
\hline
\end{tabular}




\begin{tabular}{|c|c|c|}
\hline $\begin{array}{l}\text { Allocation } \\
\text { concealment } \\
\text { (selection bias) }\end{array}$ & Unclear risk & $\begin{array}{l}\text { Not described, but implied in interactive voice response } \\
\text { system/ interactive web response system. Computer- } \\
\text { generated randomization schedule. }\end{array}$ \\
\hline $\begin{array}{l}\text { Blinding of } \\
\text { participants and } \\
\text { personnel } \\
\text { (performance bias) }\end{array}$ & Low risk & $\begin{array}{l}\text { Participants, investigators, and local sponsor personnel } \\
\text { remained blinded to treatment assignment and urine } \\
\text { samples for glucosuria until all participants completed the } \\
\text { study (week } 52 \text { visit) and the final database was locked. }\end{array}$ \\
\hline $\begin{array}{l}\text { Blinding of } \\
\text { outcome } \\
\text { assessment } \\
\text { (detection bias) }\end{array}$ & Low risk & $\begin{array}{l}\text { After randomization, A1C and FPG values and all glucose } \\
\text { levels from the FS- MMTT were masked to the study } \\
\text { centers, unless FPG/A1C values met specific study criteria } \\
\text { for discontinuation. } \\
\text { Participants, investigators, and local sponsor personnel } \\
\text { remained blinded to treatment assignment and urine } \\
\text { samples for glucosuria until all participants completed the } \\
\text { study (week } 52 \text { visit) and the final database was locked. }\end{array}$ \\
\hline $\begin{array}{l}\text { Incomplete } \\
\text { outcome data } \\
\text { (attrition bias) }\end{array}$ & Low risk & $\begin{array}{l}464 / 756 \text { completed the intervention. The primary analysis } \\
\text { was based on the modified intent- to-treat population (all } \\
\text { randomized participants who received one or more doses } \\
\text { of study drug) with a last observation carried forward } \\
\text { approach to impute missing data at the end point. } \\
\text { A higher rate of discontinuation was observed with } \\
\text { sitagliptin } 100 \mathrm{mg}(44.4 \%) \text { than with canagliflozin } 300 \mathrm{mg} \\
(32.6 \%) \text {. }\end{array}$ \\
\hline $\begin{array}{l}\text { Selective reporting } \\
\text { (reporting bias) }\end{array}$ & Low risk & All major clinically relevant outcomes were reported \\
\hline Other bias & Unclear risk & $\begin{array}{l}\text { This study was supported by Janssen Research \& } \\
\text { Development LLC, who funded editorial support. }\end{array}$ \\
\hline
\end{tabular}

Stenløf 2013

\begin{tabular}{||l|l|}
\hline Methods & $\begin{array}{l}26 \text {-week, randomized, double-blind, placebo-controlled, phase } 3 \\
\text { trial in } 17 \text { countries. }\end{array}$ \\
\hline Participants & $\begin{array}{l}587 \text { initially randomized: canagliflozin } 100 \mathrm{mg} n=195 ; \text { canagliflozin } \\
300 \mathrm{mg} n=197 ; \text { placebo } n=192 .\end{array}$ \\
\hline Interventions & $\begin{array}{l}\text { Participants in the main study were randomly assigned to receive } \\
\text { daily oral doses of canagliflozin } 100 \text { or } 300 \mathrm{mg} \text { versus placebo }(1: 1:\end{array}$ \\
\hline
\end{tabular}




\begin{tabular}{|c|c|}
\hline & $\begin{array}{l}\text { 1) once daily (oral dose) for } 26 \text { weeks. } \\
\text { Add on: diet and exercise continued during the intervention period. }\end{array}$ \\
\hline Outcomes & $\begin{array}{l}\text { Primary outcomes of the trial } \\
\text { change in HbA1c from baseline to week } 26 . \\
\text { Secondary outcomes of the trial } \\
\text { proportion of participants reaching HbA1c }<7.0 \% \text {, } \\
\text { changes from baseline at week } 26 \text { in FPG and systolic blood } \\
\text { pressure (BP), } \\
\text { percent changes from baseline in body weight, } \\
\text { high-density lipoprotein cholesterol (HDL-C) and triglycerides, } \\
\text { other fasting plasma lipids, including low-density lipoprotein } \\
\text { cholesterol (LDL-C), non - HDL-C and the LDL-C/HDL-C ratio, } \\
\text { change from baseline in apolipoprotein B (Apo B) in a subset of } \\
\text { participants in the main study (based on availability of paired } \\
\text { baseline and week } 26 \text { archive samples } \\
\text { changes in diastolic BP. } \\
\text { Adverse events and safety assessments } \\
\text { adverse event (AE) reports, } \\
\text { safety laboratory tests, } \\
\text { vital sign measurements, } \\
\text { physical examinations and } 12-l e a d \text { electrocardiograms, } \\
\text { urinary tract infections (UTIs) and genital mycotic infections, } \\
\text { documented hypoglycemia episodes, } \\
\text { severe hypoglycemia episodes. }\end{array}$ \\
\hline Inclusion criteria & $\begin{array}{l}\text { men and women } 18 \text { to } 80 \text { years of age } \\
\text { T2DM } \\
\text { not on an AHA at screening } \\
\mathrm{HbA} 1 \mathrm{c} \geq 7.0 \text { and } \leq 10.0 \% \text {, } \\
\text { on AHA monotherapy (except peroxisome proliferator-activated } \\
\text { receptor- } \gamma \text { (PPAR } \psi \text { ) agonist) or metformin plus sulfonylurea } \\
\text { combination therapy (at } \leq 50 \% \text { of maximally or near-maximally } \\
\text { effective doses) with } \mathrm{HbA} 1 \mathrm{c} \geq 6.5 \text { and } \leq 9.5 \% \text { at screening and } \\
\mathrm{HbA} 1 \mathrm{c} \geq 7.0 \text { and } \leq 10.0 \% \text { and fasting plasma glucose (FPG) }<15.0 \\
\mathrm{mmol} / \mathrm{I} \text { at week }-2 \text {. }\end{array}$ \\
\hline Exclusion criteria & $\begin{array}{l}\text { repeated FPG measurements }>15.0 \mathrm{mmol} / / \text { during the pre- } \\
\text { treatment phase (or }>19.4 \mathrm{mmol} / \mathrm{l} \text { for the high glycemic sub study), } \\
\text { type } 1 \text { diabetes, } \\
\text { hereditary glucose-galactose malabsorption, }\end{array}$ \\
\hline
\end{tabular}




\begin{tabular}{|c|c|}
\hline & $\begin{array}{l}\text { primary renal glucosuria, } \\
\text { cardiovascular (CV) disease (including myocardial infarction, } \\
\text { unstable angina, revascularization procedure or cerebrovascular } \\
\text { accident), } \\
\text { treatment with a PPARy agonist, insulin, another SGLT2 inhibitor or } \\
\text { any other AHA except as specified in the inclusion criteria within12 } \\
\text { weeks before screening, } \\
\text { eGFR }<50 \mathrm{ml} / \mathrm{min} / 1.73 \mathrm{~m}^{2} \text { at screening. }\end{array}$ \\
\hline Notes & $\begin{array}{l}\text { ClinicalTrials.gov identifier: NCT01081834 } \\
\text { In this review, only the main placebo controlled study contributed } \\
\text { data to our analyses, i.e. the study continued for } 26 \text { weeks (single } \\
\text { blind) after initial intervention period, this review reports only } \\
\text { outcomes from the initial } 26 \text {-week double blind intervention. (An } \\
\text { active-controlled, } 26 \text {-week extension (blinded switch of placebo- } \\
\text { treated patients to sitagliptin } 100 \text { mg (placebo/sitagliptin)) (CANA } \\
\text { Stenløf 2014). } \\
\text { "For subjects with HbA1c values above the inclusion range (HbA1c } \\
\geq 7.0 \text { and } \leq 10.0 \% \text { ), a sub study was conducted to assess the efficacy } \\
\text { in elevated glycemic states. Subjects were eligible to participate in } \\
\text { the high glycemic sub study if they had HbA1c }>10.0 \text { and }<12.0 \% \text { at } \\
\text { screening or week }-1 \text { and FPG }>19.4 \text { mmol/l at week }-1 \text {. Subjects } \\
\text { eligible for this sub study entered a } 1 \text {-week, single-blind, placebo } \\
\text { run-in period followed by a } 26 \text {-week, double-blind, active-treatment } \\
\text { period. Given the poorer glycemic control, all subjects received } \\
\text { active treatment with canagliflozin } 100 \text { or } 300 \text { mg; double-blinding } \\
\text { was to the dose of canagliflozin. Subjects in the high glycemic sub } \\
\text { study were not eligible for the } 26 \text {-week extension period. In this } \\
\text { report, the placebo-controlled study component will be referred to } \\
\text { as the 'main study'." }\end{array}$ \\
\hline
\end{tabular}

\section{Risk of bias table}

\begin{tabular}{||l||l|l||}
\hline Bias & $\begin{array}{l}\text { Authors' } \\
\text { judgement }\end{array}$ & Support for judgement \\
\hline $\begin{array}{l}\text { Random sequence } \\
\text { generation } \\
\text { (selection bias) }\end{array}$ & Unclear risk & Not described \\
\hline $\begin{array}{l}\text { Allocation } \\
\text { concealment }\end{array}$ & Unclear risk & Not described \\
\hline
\end{tabular}




\begin{tabular}{|c|c|c|}
\hline (selection bias) & & \\
\hline $\begin{array}{l}\text { Blinding of } \\
\text { participants and } \\
\text { personnel } \\
\text { (performance bias) }\end{array}$ & Low risk & Described as double-blind \\
\hline $\begin{array}{l}\text { Blinding of } \\
\text { outcome } \\
\text { assessment } \\
\text { (detection bias) }\end{array}$ & Low risk & Described as double-blind \\
\hline $\begin{array}{l}\text { Incomplete } \\
\text { outcome data } \\
\text { (attrition bias) }\end{array}$ & Low risk & $\begin{array}{l}584 / 587 \text { completed the intervention i.e. they received at } \\
\text { least one dose of the study medication. } \\
\text { Efficacy and safety analyses for the main study and the } \\
\text { high glycemic sub study were performed separately using } \\
\text { the modified intent-to-treat (mITT) population consisting } \\
\text { of all randomized participants who received } \geq 1 \text { dose of } \\
\text { the study drug. The last observation carried forward } \\
\text { (LOCF) approach was used to impute missing efficacy } \\
\text { data. For participants who received rescue therapy, the } \\
\text { last post-baseline value prior to the initiation of rescue } \\
\text { therapy was used for the efficacy analyses. } \\
\text { All drops outs and losses to follow up accounted for. } \\
\text { Slightly higher rates of discontinuation were seen with } \\
\text { placebo. }\end{array}$ \\
\hline $\begin{array}{l}\text { Selective reporting } \\
\text { (reporting bias) }\end{array}$ & Low risk & $\begin{array}{l}\text { Some additional outcomes, besides the pre-specified ones } \\
\text { were reported. But all major clinically relevant pre- } \\
\text { specified outcomes reported. }\end{array}$ \\
\hline Other bias & Unclear risk & $\begin{array}{l}\text { This study was sponsored by Janssen Research \& } \\
\text { Development, LLC who provided assistance and } \\
\text { contribution to the clinical management, data review and } \\
\text { preparation of the study report. }\end{array}$ \\
\hline
\end{tabular}

\section{Strojek 2011}

Methods 24-week randomized, double-blind, parallel-group, placebocontrolled, multicenter trial at 84 sites in the Czech Republic (11 centers), Hungary (16), Republic of Korea (12), Philippines (5), Poland (29), Thailand (3) and Ukraine (8) 


\begin{tabular}{|c|c|}
\hline Participants & $\begin{array}{l}597 \text { randomized to study drug + glimepiride } 4 \text { mg/day. } 146 \text { placebo; } \\
154 \text { dapagliflozin } 2.5 \mathrm{mg} / \text { day; } 145 \text { dapagliflozin } 5 \mathrm{mg} / \text { day; } 151 \\
\text { dapagliflozin } 10 \mathrm{mg} / \text { day. }\end{array}$ \\
\hline Interventions & $\begin{array}{l}\text { Randomized to receive double- blind dapagliflozin } 2.5,5 \text { or } 10 \mathrm{mg} \text { or } \\
\text { placebo taken orally once per day before the first meal of the day } \\
\text { and added to continuing open-label glimepiride } 4 \mathrm{mg} / \mathrm{day} \text {. } \\
\text { Add on: glimepiride and rescue therapy were administered as open } \\
\text { label treatments for } 24 \text { weeks with a } 24 \text {-weeks double blind } \\
\text { extension period. }\end{array}$ \\
\hline Outcomes & $\begin{array}{l}\text { Primary outcomes of the trial } \\
\text { The primary endpoint was change in central laboratory HbA1c } \\
\text { percentage from baseline to week } 24, \\
\text { Secondary outcomes of the trial } \\
\text { change in total body weight (TBW) from baseline to week } 24 \\
\text { change from baseline to week } 24 \text { in } 2 \text {-h post-challenge plasma } \\
\text { glucose (PPG) rise in response to an oral glucose-tolerance test } \\
\text { (OGTT) using } 75 \text { g of glucose } \\
\text { proportion of patients achieving a therapeutic } \\
\text { glycemic response, defined as HbA1c< } 7 \% \text { at week } 24 \\
\text { change in TBW from baseline to week } 24 \text { in patients } \\
\text { with baseline BMI } 227 \text { kg/m2 } \\
\text { change in FPG from } \\
\text { baseline to week } 24 \text {. } \\
\text { proportions of patients receiving rescue therapy for failing to reach } \\
\text { prespecified glycemic targets or discontinuing for lack of efficacy, } \\
\text { seated systolic and diastolic blood pressure } \\
\text { lipids } \\
\text { Adverse events and safety assessments } \\
\text { adverse events } \\
\text { hypoglycemic events, } \\
\text { laboratory tests, } \\
\text { electrocardiographic and physical examinations } \\
\text { vital signs (including orthostatic hypotension). } \\
\text { genital infection }\end{array}$ \\
\hline Inclusion criteria & $\begin{array}{l}\text { men and women } \\
\text { aged } \geq 18 \text { years }\end{array}$ \\
\hline
\end{tabular}




\begin{tabular}{|c|c|}
\hline & $\begin{array}{l}\text { inadequately controlled T2DM, } \\
\mathrm{HbA} 1 \mathrm{c} \geq 7 \text { and } \leq 10 \% \text {, who were receiving a stable dose of } \\
\text { sulphonylurea monotherapy that was at a dose level of at least half } \\
\text { the maximum recommended for at least } 8 \text { weeks prior to } \\
\text { enrolment. } \\
\text { fasting plasma } \\
\text { glucose (FPG) }>15 \mathrm{mmol} / \mathrm{l} \text { and fasting C-peptide } \geq 0.33 \mathrm{nmol} / \mathrm{l} \text {. }\end{array}$ \\
\hline Exclusion criteria & $\begin{array}{l}\text { type } 1 \text { diabetes, } \\
\text { diabetes insipidus, } \\
\text { corticosteroid-induced type } 2 \text { diabetes, } \\
\text { a history of diabetic ketoacidosis or hyperosmolar non-ketotic } \\
\text { coma, } \\
\text { poorly controlled diabetes characterized by polyuria/polydipsia } \\
\text { with }>10 \% \text { weight loss, } \\
\text { use of insulin for }>7 \text { consecutive days during the } 24 \text { weeks prior to } \\
\text { enrolment, } \\
\text { and use of glimepiride }>4 \mathrm{mg} / \text { day during the } 8 \text { weeks up to and } \\
\text { including enrolment, } \\
\text { body mass index (BMI) }>45.0 \mathrm{~kg} / \mathrm{m}^{2} \text {, } \\
\text { calculated creatinine clearance }<50 \mathrm{~mL} / \mathrm{min} \text { or serum creatinine } \\
>177 \mu \text { mol/L, } \\
\text { urine albumin/creatinine ratio }>203.4 \mathrm{mg} / \mathrm{mmol} \text {, } \\
\text { aspartate aminotransferase and/or alanine aminotransferase } \\
\text { and/or creatine kinase } \geq 3 \times \text { upper limit of normal range, } \\
\text { serum total bilirubin }>34 \mu \mathrm{mol} / \mathrm{L} \text {; } \mathrm{Hb} \leq 10 \mathrm{~g} / \mathrm{dL} \text { for men and } \leq 9.5 \\
\text { g/dL for women, } \\
\text { systolic blood pressure } \geq 180 \mathrm{mmHg} \text { and/or diastolic blood pressure } \\
\geq 110 \text { mmHg, } \\
\text { cardiovascular event within } 6 \mathrm{months} \text { of enrolment, } \\
\text { congenital renal glycosuria, } \\
\text { significant renal, hepatic, hematological, oncological, endocrine, } \\
\text { immunological (including hypersensitivity to study medications), } \\
\text { psychiatric disease (including alcohol and substance misuse), } \\
\text { pregnancy or lactation, } \\
\text { use of systemic corticosteroids for }>4 \text { weeks within } 3 \text { months of } \\
\text { enrolment, } \\
\text { weight loss medication within } 30 \text { days of enrolment. }\end{array}$ \\
\hline Notes & $\begin{array}{l}\text { ClinicalTrials.gov identifier: NCT00680745 } \\
\text { Data from the } 24 \text {-week double-blind extension period Strojek } 2014 \\
\text { were included in our analyses. } \\
\text { Demographic and baseline characteristics were balanced across } \\
\text { treatment groups, with } 30.6 \% \text { of patients recruited from the }\end{array}$ \\
\hline
\end{tabular}


Risk of bias table

\begin{tabular}{|c|c|c|}
\hline Bias & $\begin{array}{l}\text { Authors' } \\
\text { judgement }\end{array}$ & Support for judgement \\
\hline $\begin{array}{l}\text { Random sequence } \\
\text { generation } \\
\text { (selection bias) }\end{array}$ & Low risk & $\begin{array}{l}\text { A computer-generated randomization schedule was } \\
\text { provided by AstraZeneca using blocks to balance the } \\
\text { treatment groups in a } 1: 1: 1: 1 \text { ratio. Patients were } \\
\text { randomized strictly sequentially at each center. }\end{array}$ \\
\hline $\begin{array}{l}\text { Allocation } \\
\text { concealment } \\
\text { (selection bias) }\end{array}$ & Unclear risk & $\begin{array}{l}\text { Not described in detail but implied by computer- } \\
\text { generated randomization schedule using block } \\
\text { randomization. }\end{array}$ \\
\hline $\begin{array}{l}\text { Blinding of } \\
\text { participants and } \\
\text { personnel } \\
\text { (performance bias) }\end{array}$ & Low risk & $\begin{array}{l}\text { Blinding of dapagliflozin tablets was achieved by double- } \\
\text { blind allocation and use of a double- dummy technique } \\
\text { because the dapagliflozin } 10 \mathrm{mg} \text { tablet size was slightly } \\
\text { larger than that for the } 2.5 \text { and } 5 \mathrm{mg} \text { doses. }\end{array}$ \\
\hline $\begin{array}{l}\text { Blinding of } \\
\text { outcome } \\
\text { assessment } \\
\text { (detection bias) }\end{array}$ & Low risk & $\begin{array}{l}\text { Double blind. double-blind allocation and use of a double } \\
\text { dummy technique because the dapagliflozin } 10 \mathrm{mg} \text { tablet } \\
\text { size was slightly larger than that for the } 2.5 \text { and } 5 \mathrm{mg} \\
\text { doses. }\end{array}$ \\
\hline $\begin{array}{l}\text { Incomplete } \\
\text { outcome data } \\
\text { (attrition bias) }\end{array}$ & Low risk & $\begin{array}{l}546 / 597 \text { completed. Two analysis sets were defined: the } \\
\text { safety analysis set consisting of all patients who received } \\
\geq 1 \text { dose of study medication and the full analysis set } \\
\text { consisting of all randomized patients who received } \geq 1 \\
\text { dose of study medication and who had a non-missing } \\
\text { baseline and } \geq 1 \text { post-baseline efficacy value for } \geq 1 \\
\text { efficacy variable. Primary, secondary and exploratory } \\
\text { endpoints were analyzed with the full analysis set. } \\
\text { Observations after initiation of rescue therapy were } \\
\text { excluded, and these and other missing values were } \\
\text { replaced using the LOCF method. The majority of } \\
\text { randomized patients completed the study (91.5\%), All } \\
\text { dropouts and lost to follow-up accounted for. }\end{array}$ \\
\hline $\begin{array}{l}\text { Selective reporting } \\
\text { (reporting bias) }\end{array}$ & Low risk & $\begin{array}{l}\text { Besides the major prespecified outcomes, a number of } \\
\text { exploratory endpoints were assessed, which included } \\
\text { proportions of patients receiving rescue therapy for failing } \\
\text { to reach prespecified glycemic targets or discontinuing for }\end{array}$ \\
\hline
\end{tabular}




\begin{tabular}{||l||l|l||}
\hline \hline & $\begin{array}{l}\text { lack of efficacy, seated systolic and diastolic blood } \\
\text { pressure and lipid parameters. }\end{array}$ \\
\hline Other bias & Unclear risk & $\begin{array}{l}\text { Medical writing and editorial was funded by AstraZeneca } \\
\text { and Bristol-Myers Squibb. Some of the trialists are } \\
\text { employees of AstraZeneca. Data analysis was conducted } \\
\text { by a company which is contracted to support data analysis } \\
\text { for AstraZeneca. }\end{array}$ \\
\hline
\end{tabular}

Wilding 2009

\begin{tabular}{|c|c|}
\hline Methods & $\begin{array}{l}\text { 12-week randomized, double-blind, three arm parallel-group, } \\
\text { placebo-controlled, 26-center trial (U.S. and Canada). }\end{array}$ \\
\hline Participants & $\begin{array}{l}71 \text { initially randomized: placebo } n=23 ; 10 m g \text { dapagliflozin } n=24 ; 20 \\
\text { mg dapagliflozin } n=24\end{array}$ \\
\hline Interventions & $\begin{array}{l}\text { Randomly assigned } 1: 1: 1 \text { on day } 1 \text { to double-blind placebo, } 10 \mathrm{mg} \\
\text { dapagliflozin, or } 20 \mathrm{mg} \text { dapagliflozin once daily, in addition to open- } \\
\text { label therapy with } 50 \% \text { of their usual daily insulin dose and their } \\
\text { OAD(s). } \\
\text { Add on: metformin, insulin, pioglitazone, rosiglitazon }\end{array}$ \\
\hline Outcomes & $\begin{array}{l}\text { Primary outcomes of the trial } \\
\text { change from baseline in A1C at week } 12 \\
\text { Secondary outcomes of the trial } \\
\text { changes from baseline in FPG } \\
\text { total daily dose of insulin (TDDI), } \\
\text { proportion achieving a decrease in A1C } 0.5 \% \text { from baseline, } \\
\text { proportion patients achieving A1C } 7 \% \text {. } \\
\text { total body weight } \\
\text { postprandial glucose (PPG) measured by an oral glucose tolerance } \\
\text { test. } \\
\text { Adverse events and safety assessments } \\
\text { emergent adverse events, } \\
\text { vital signs, } \\
\text { laboratory measurements, including } 24-h \text { urine collections for } \\
\text { volume and electrolytes. }\end{array}$ \\
\hline Inclusion criteria & men and women \\
\hline
\end{tabular}




\begin{tabular}{|c|c|}
\hline & $\begin{array}{l}\text { with type } 2 \text { diabetes, } \\
\text { aged } 18-75 \text { years } \\
\text { with } \mathrm{BMI} \leq 45 \mathrm{~kg} / \mathrm{m}^{2} \\
\text { A1C } 7.5-10 \% \text {, } \\
\text { stable-dose insulin sensitizer therapy (metformin 1,000 mg and/or } \\
\text { pioglitazone } 30 \mathrm{mg} \text { or rosiglitazone } 4 \mathrm{mg} \text { ) for } 6 \text { weeks and insulin } \\
\text { therapy for } 12 \text { weeks before enrolment } \\
\text { fasting C-peptide } 0.8 \mathrm{ng} / \mathrm{ml} \text {, } \\
\text { serum creatinine } 1.5 \mathrm{mg} / \mathrm{dl} \text { (men) or } 1.4 \mathrm{mg} / \mathrm{dl} \text { (women) } \\
\text { urine microalbumin-to creatinine ratio } 300 \mathrm{mg} / \mathrm{g} \\
24-\mathrm{h} \text { urine total protein } 3 \mathrm{~g} / 24 \mathrm{~h} \text {. }\end{array}$ \\
\hline Exclusion criteria & $\begin{array}{l}\text { type } 1 \text { diabetes, } \\
\text { aspartate transaminase and/or alanine transaminase } 2.5 \text { times the } \\
\text { upper limits of normal, } \\
\text { creatine kinase } 3 \text { times the upper limits of normal, } \\
\text { symptoms of severely uncontrolled diabetes, } \\
\text { history of severe hypoglycemia, } \\
\text { unstable condition or serious cardiovascular, renal, or hepatic } \\
\text { disease. }\end{array}$ \\
\hline Notes & $\begin{array}{l}\text { No ClinicalTrials.gov identifier. } \\
\text { Receiving high doses of insulin and insulin sensitizers }\end{array}$ \\
\hline
\end{tabular}

Risk of bias table

\begin{tabular}{||l||l||l||}
\hline Bias & $\begin{array}{l}\text { Authors' } \\
\text { judgement }\end{array}$ & Support for judgement \\
\hline $\begin{array}{l}\text { Random sequence } \\
\text { generation (selection } \\
\text { bias) }\end{array}$ & Unclear risk & Randomly assigned, but no details provided. \\
\hline $\begin{array}{l}\text { Allocation } \\
\text { concealment } \\
\text { (selection bias) }\end{array}$ & Unclear risk & No details provided. \\
\hline $\begin{array}{l}\text { Blinding of } \\
\text { participants and } \\
\text { personnel } \\
\text { (performance bias) }\end{array}$ & Low risk & Double blind placebo. \\
\hline $\begin{array}{l}\text { Blinding of outcome } \\
\text { assessment (detection }\end{array}$ & Low risk & Double blind placebo. \\
\hline
\end{tabular}




\begin{tabular}{|l||l||l||}
\hline bias) & & \\
\hline $\begin{array}{l}\text { Incomplete outcome } \\
\text { data (attrition bias) }\end{array}$ & Low risk & $\begin{array}{l}\text { 60/71 completed. Last observation carried forward. } \\
\text { The primary efficacy dataset consisted of all randomly } \\
\text { assigned patients who took 1 dose of double-blind } \\
\text { study medication. All dropouts and lost to follow-up } \\
\text { accounted for. }\end{array}$ \\
\hline $\begin{array}{l}\text { Selective reporting } \\
\text { (reporting bias) }\end{array}$ & Low risk & $\begin{array}{l}\text { All clinically relevant outcomes are defined and } \\
\text { reported. }\end{array}$ \\
\hline Other bias & Unclear risk & $\begin{array}{l}\text { This study was funded by Bristol-Myers Squibb and } \\
\text { AstraZeneca. Bristol-Myers Squibb provided writing } \\
\text { and editorial support. }\end{array}$ \\
\hline
\end{tabular}

\section{Wilding 2012}

\begin{tabular}{|c|c|}
\hline Methods & $\begin{array}{l}\text { 48-week, randomized, placebo-controlled, parallel group, } \\
\text { multicenter trial } 126 \text { centers worldwide (Austria, Bulgaria, Canada, } \\
\text { Finland, Great Britain, hungry, the Netherlands, Romania, Russian } \\
\text { Federation, Slovakia, Spain, United States). }\end{array}$ \\
\hline Participants & $\begin{array}{l}808 \text { initially randomized: placebo plus insulin } n=193 \text {; dapagliflozin } \\
2.5 \text { mg plus insulin } N=202 ; \text { dapagliflozin } 5 \text { mg plus insulin } n=211 ; \\
\text { dapagliflozin } 10 \text { mg plus insulin } n=194\end{array}$ \\
\hline Interventions & $\begin{array}{l}\text { Randomly assigned on a 1:1:1:1 basis to receive placebo or } 2.5,5 \text {, or } \\
10 \text { mg of dapagliflozin, once daily, for } 24 \text { weeks, extended to } 48 \\
\text { weeks. } \\
\text { Add on: open-label therapy with their usual daily dose of insulin and } \\
\text { existing oral antidiabetic drugs (OADs). } \\
\text { No rescue medication reported. }\end{array}$ \\
\hline Outcomes & $\begin{array}{l}\text { Primary outcomes of the trial } \\
\text { change in HBA1c from baseline to } 48 \text { weeks } \\
\text { Secondary outcomes of the trial } \\
\text { bodyweight, } \\
\text { insulin dose, } \\
\text { fasting plasma glucose level at } 24 \text { weeks } \\
\text { Adverse events and safety assessments }\end{array}$ \\
\hline
\end{tabular}




\begin{tabular}{|c|c|}
\hline & $\begin{array}{l}\text { adverse events, } \\
\text { laboratory variables, } \\
\text { vital signs, } \\
\text { hyperglycemia, } \\
\text { genital infection, } \\
\text { urinary tract infection (UTI) }\end{array}$ \\
\hline Inclusion criteria & $\begin{array}{l}\text { men and women } \\
\text { aged } 18 \text { to } 80 \text { years } \\
\text { T2DM } \\
\text { BMI } \leq 45 \mathrm{~kg} / \mathrm{m}^{2} \\
\text { inadequate glycemic control ( } \mathrm{HbA} 1 \mathrm{c} \geq 7.5 \% \text { and } \leq 10.5 \% \text { ). } \\
\text { Participants had to have received a stable insulin regimen with a } \\
\text { mean daily insulin dose of } 30 \mathrm{U} \text { or more for at least } 8 \text { weeks, with } \\
\text { daily insulin requirements varying by more than } 10 \% \text { on no more } \\
\text { than } 1 \text { occasion in the } 7 \text { days before randomization. }\end{array}$ \\
\hline Exclusion criteria & $\begin{array}{l}\text { Type I diabetes mellitus, } \\
\text { symptoms of poorly controlled diabetes, } \\
\text { calculated creatinine clearance less than } 50 \mathrm{~mL} \text { per minute per } \\
1.73 \mathrm{~m}^{2} \text {, or measured serum creatinine level greater than hundred } \\
\text { and } 77 \mu \mathrm{mol} \text { per liter }(>2 \mathrm{mg} / \mathrm{dL} \text { ) or if receiving metformin greater } \\
\text { than } 133 \mu \mathrm{mol} \text { per liter }(>1.5 \mathrm{micrograms} \text { per deciliter) for men and } \\
\text { at least } 124 \mu \mathrm{mol} \text { per liter }(\geq 1.4 \mathrm{mcg} / \mathrm{dL} \text { ) for women }\end{array}$ \\
\hline \multirow[t]{2}{*}{ Notes } & ClinicalTrials.gov identifier: NCT00673231 \\
\hline & $\begin{array}{l}\text { We included the 24-week extension study results (Wilding 2014) in } \\
\text { our analyses (i.e. 48-week outcome data). }\end{array}$ \\
\hline
\end{tabular}

Risk of bias table

\begin{tabular}{||l|l|l||}
\hline Bias & $\begin{array}{l}\text { Authors' } \\
\text { judgement }\end{array}$ & Support for judgement \\
\hline $\begin{array}{l}\text { Random sequence } \\
\text { generation } \\
\text { (selection bias) }\end{array}$ & Low risk & $\begin{array}{l}\text { A computer-generated, stratified, block-randomization } \\
\text { schedule containing stratum, randomization code, and } \\
\text { treatment was provided by AstraZeneca. }\end{array}$ \\
\hline $\begin{array}{l}\text { Allocation } \\
\text { concealment } \\
\text { (selection bias) }\end{array}$ & Unclear risk & $\begin{array}{l}\text { Not described in detail but implied in computer-generated } \\
\text { randomization. }\end{array}$ \\
\hline Blinding of & Low risk & Patients, investigators, study monitors, and personnel at \\
\hline
\end{tabular}




\begin{tabular}{|c|c|c|}
\hline $\begin{array}{l}\text { participants and } \\
\text { personnel } \\
\text { (performance bias) }\end{array}$ & & $\begin{array}{l}\text { AstraZeneca and Bristol-Myers Squibb had no access to } \\
\text { the randomization scheme, except for cases of medical } \\
\text { emergencies. }\end{array}$ \\
\hline $\begin{array}{l}\text { Blinding of } \\
\text { outcome } \\
\text { assessment } \\
\text { (detection bias) }\end{array}$ & Unclear risk & $\begin{array}{l}\text { Because primary efficacy analyses were planned at } 24 \\
\text { weeks, personnel at AstraZeneca and Bristol- Myers } \\
\text { Squibb had access to the data at that time. During the } \\
\text { double-blind extension periods, investigators, patients, } \\
\text { and study monitors remained blinded, except for cases of } \\
\text { medical emergencies. }\end{array}$ \\
\hline $\begin{array}{l}\text { Incomplete } \\
\text { outcome data } \\
\text { (attrition bias) }\end{array}$ & Low risk & $\begin{array}{l}\text { Two analysis sets were defined: the safety set, comprising } \\
\text { all randomly assigned patients who received at least } 1 \\
\text { dose of study medication, and the full set, comprising all } \\
\text { randomly assigned patients who received at least } 1 \text { dose } \\
\text { of study medication and had a non-missing baseline value } \\
\text { and at least } 1 \text { post-baseline efficacy value for at least } 1 \\
\text { efficacy variable. Efficacy variables were analyzed with the } \\
\text { full analysis set. Completers PLA: } 157 / 197 \text {, Dapagliflozin } \\
10 \mathrm{mg} 170 / 196 \text {. }\end{array}$ \\
\hline $\begin{array}{l}\text { Selective reporting } \\
\text { (reporting bias) }\end{array}$ & Low risk & All major clinically relevant outcomes have been reported. \\
\hline Other bias & Unclear risk & $\begin{array}{l}\text { Sponsored by AstraZeneca and Bristol Myers squib. } \\
\text { Sponsors involved in the study design, data collection, } \\
\text { review and analysis, editorial assistants funded by the } \\
\text { sponsors. }\end{array}$ \\
\hline
\end{tabular}

\section{Wilding 2013}

\begin{tabular}{||l|l||}
\hline Methods & $\begin{array}{l}78 \text { week randomized, double-blind, placebo-controlled, multicenter } \\
\text { study at } 85 \text { study centers in } 11 \text { countries followed by a 26-week, } \\
\text { double-blind, extension period. }\end{array}$ \\
\hline Participants & $\begin{array}{l}469 \text { initially randomized: placebo } n=156 ; 100 \text { mg canagliflozin } \\
n=157 ; \text { canagliflozin } n=156\end{array}$ \\
\hline Interventions & $\begin{array}{l}1: 1: 1 \text { ratio to receive canagliflozin } 100 \text { or } 300 \text { mg or placebo once } \\
\text { daily (oral dose) before the first meal of the day for } 26 \text { weeks } \\
\text { (extended to } 52 \text { weeks). } \\
\text { Add on: metformin, sulfonylurea }\end{array}$ \\
\hline Outcomes & \begin{tabular}{l} 
Primary outcomes of the trial \\
\hline
\end{tabular} \\
\hline
\end{tabular}




\begin{tabular}{|c|c|}
\hline & $\begin{array}{l}\text { change from baseline in HbA1c at week } 52 . \\
\text { Secondary outcomes of the trial } \\
\text { proportion of patients achieving HbA1c <7.0\%, } \\
\text { change from baseline in FPG and systolic BP, } \\
\text { per cent change from baseline in body weight, } \\
\text { high density lipoprotein cholesterol (HDL-C), and triglycerides. } \\
\text { Homeostasis Model Assessment (HOMA2-\% B), } \\
\text { a fasting measure of } \beta \text {-cell function, based on FPG and C-peptide } \\
\text { measurements, } \\
\text { in subset of patients: FS-MMTT on day } 1 \text { and at week 26, 2-h } \\
\text { postprandial glucose (PPG), glucose area under the concentration- } \\
\text { time curve (AUCG), incremental AUCG (\#AUCG), and the ratio of C- } \\
\text { peptide AUC (AUCC) to AUCG were assessed. } \\
\text { Adverse events and safety assessments } \\
\text { adverse event (AE) reports over the 52-week period, } \\
\text { safety laboratory tests, } \\
\text { vital sign measurements, } \\
12 \text {-lead electrocardiograms, } \\
\text { physical examinations, } \\
\text { genital mycotic infections and urinary tract infections (UTIs), } \\
\text { hypoglycemia events. }\end{array}$ \\
\hline Inclusion criteria & $\begin{array}{l}\text { men and women } \\
\text { aged } 18-80 \text { years } \\
\text { T2DM } \\
\text { inadequate glycemic control ( } \mathrm{HbA} 1 \mathrm{c} \geq 7.0 \% \text { to } \leq 10.5 \% \text { ), } \\
\text { on metformin plus sulphonylurea, with both agents at maximally or } \\
\text { near-maximally effective doses. }\end{array}$ \\
\hline Exclusion criteria & $\begin{array}{l}\text { history of diabetic ketoacidosis, } \\
\text { T1DM } \\
\text { repeated fasting plasma glucose (FPG) } \geq 15.0 \mathrm{mmol} / \mathrm{I} \text { during the } \\
\text { pretreatment phase, } \\
\text { history of at least } 1 \text { severe hypoglycemia episode within } 6 \text { months } \\
\text { before screening, } \\
\text { eGFR }<55 \mathrm{ml} / \mathrm{min} / 1.73 \mathrm{~m} 2 \text { (or }<60 \mathrm{ml} / \mathrm{min} / 1.73 \mathrm{~m}^{2} \text { based upon } \\
\text { restriction of metformin use in the local label) or serum creatinine } \\
\geq 124 \mu \mathrm{mol} / \mathrm{I} \text { for men and } \geq 115 \mu \mathrm{mol} / \mathrm{l} \text { for women, } \\
\text { uncontrolled hypertension (systolic } \mathrm{BP} \geq 160 \mathrm{mmHg} \text { or diastolic BP } \\
\geq 100 \mathrm{mmHg} \text { ), } \\
\text { taking any ant hyperglycemic agent other than metformin plus }\end{array}$ \\
\hline
\end{tabular}


sulphonylurea within 12 weeks prior to screening.

Notes

ClinicalTrials.gov identifier: NCT01106625

In this review, data from the 26 week 'core' period and the extension period (52 weeks) contributed data to our analyses.

\section{Risk of bias table}

\begin{tabular}{|c|c|c|}
\hline Bias & \begin{tabular}{|l|} 
Authors' \\
judgement
\end{tabular} & Support for judgement \\
\hline $\begin{array}{l}\text { Random sequence } \\
\text { generation } \\
\text { (selection bias) }\end{array}$ & Low risk & $\begin{array}{l}\text { Randomization was performed using an Interactive Voice } \\
\text { Response System/Interactive Web Response System based } \\
\text { on a computer-generated schedule prepared by the } \\
\text { sponsor before the study. }\end{array}$ \\
\hline $\begin{array}{l}\text { Allocation } \\
\text { concealment } \\
\text { (selection bias) }\end{array}$ & Unclear risk & $\begin{array}{l}\text { Randomization was performed using an Interactive Voice } \\
\text { Response System/Interactive Web Response System based } \\
\text { on a computer-generated schedule. }\end{array}$ \\
\hline $\begin{array}{l}\text { Blinding of } \\
\text { participants and } \\
\text { personnel } \\
\text { (performance bias) }\end{array}$ & Low risk & $\begin{array}{l}\text { To maintain blinding after randomization, } \mathrm{HbA} 1 \mathrm{c} \text { and FPG } \\
\text { values were masked to study centers unless these values } \\
\text { met prespecified glycemic rescue criteria or after glycemic } \\
\text { rescue therapy was started. Patients, investigators and } \\
\text { local sponsor personnel remained blinded throughout the } \\
\text { core study and extension period. }\end{array}$ \\
\hline $\begin{array}{l}\text { Blinding of } \\
\text { outcome } \\
\text { assessment } \\
\text { (detection bias) }\end{array}$ & Unclear risk & $\begin{array}{l}\text { After completion of the core treatment period, the } \\
\text { database was locked and the study was unblinded by the } \\
\text { sponsor for regulatory filing; patients, investigators and } \\
\text { local sponsor personnel remained blinded throughout the } \\
\text { extension period. }\end{array}$ \\
\hline $\begin{array}{l}\text { Incomplete } \\
\text { outcome data } \\
\text { (attrition bias) }\end{array}$ & Low risk & $\begin{array}{l}\text { 264/469 randomized completed i.e. took at least } 1 \text { dose of } \\
\text { double- blind study drug. Primary efficacy analyses were } \\
\text { conducted using the modified intent-to-treat (mITT) } \\
\text { population (all randomized patients who took } 1 \text { dose of } \\
\text { double- blind study drug. Efficacy data were analyzed } \\
\text { according to randomized treatment with the last } \\
\text { observation carried forward (LOCF) approach used to } \\
\text { impute missing values. }\end{array}$ \\
\hline Selective reporting & Low risk & Major clinically relevant outcomes reported. \\
\hline
\end{tabular}




\begin{tabular}{|l||l||l||}
\hline (reporting bias) & \multicolumn{1}{||l||}{} & \\
\hline \hline Other bias & Unclear risk & $\begin{array}{l}\text { This study was supported by Janssen Research \& } \\
\text { Development, LLC who provided assistance in clinical } \\
\text { review of patient safety and adherence to the study } \\
\text { protocol and contributions to the analysis and } \\
\text { interpretation of study data and editorial support. }\end{array}$ \\
\hline
\end{tabular}

\section{Footnotes}

eGFR: estimated glomerular filtration rate

T2DM: Type 2 diabetes mellitus

T1DM : type 1 diabetes mellitus

BMI: body mass index

T2DM: type 2 diabetes mellitus

HbA1c: glycated hemoglobin

OAD: oral antidiabetic

SU: sulphonylurea 